\title{
Prevention of pressure ulcers in nursing homes, a big challenge
}

Citation for published version (APA):

van Leen, M. W. F. (2017). Prevention of pressure ulcers in nursing homes, a big challenge. [Doctoral Thesis, Maastricht University]. Maastricht University. https://doi.org/10.26481/dis.20170413mvl

Document status and date:

Published: 01/01/2017

DOI:

10.26481/dis.20170413mvl

Document Version:

Publisher's PDF, also known as Version of record

\section{Please check the document version of this publication:}

- A submitted manuscript is the version of the article upon submission and before peer-review. There can be important differences between the submitted version and the official published version of record.

People interested in the research are advised to contact the author for the final version of the publication, or visit the DOI to the publisher's website.

- The final author version and the galley proof are versions of the publication after peer review.

- The final published version features the final layout of the paper including the volume, issue and page numbers.

Link to publication

\footnotetext{
General rights rights.

- You may freely distribute the URL identifying the publication in the public portal. please follow below link for the End User Agreement:

www.umlib.nl/taverne-license

Take down policy

If you believe that this document breaches copyright please contact us at:

repository@maastrichtuniversity.nl

providing details and we will investigate your claim.
}

Copyright and moral rights for the publications made accessible in the public portal are retained by the authors and/or other copyright owners and it is a condition of accessing publications that users recognise and abide by the legal requirements associated with these

- Users may download and print one copy of any publication from the public portal for the purpose of private study or research.

- You may not further distribute the material or use it for any profit-making activity or commercial gain

If the publication is distributed under the terms of Article $25 \mathrm{fa}$ of the Dutch Copyright Act, indicated by the "Taverne" license above, 
PreVENTION OF PRESSURE ULCERS IN NURSING HOMES, A BIG CHALLENGE

\section{MARTIN VAN LEEN}


Prevention of pressure ulcers in nursing homes, a big challenge

Copyright MWF van Leen @ 2017

All rights reserved. No part of this publication may be reproduced, or transmitted in any form or any means, electronic or mechanical, including photocopying, recording, or any information storage and retrieval without prior written permission from the author, or, when appropriate the publishers of the papers.

Niets uit deze uitgave mag worden verveelvoudigd en/of openbaar gemaakt worden door middel van druk, fotokopie, microfilm of enige andere wijze, noch opgeslagen worden in een retrieval-systeem noch vertaald worden in welke taal dan ook, zonder schriftelijke toestemming van de auteur.

\section{Colofon}

Cover design: Dirk Franssens, huisarts te Dordrecht

Layout \& production: Optima Grafische Communicatie

ISBN / EAN: 978-94-92683-10-6 


\title{
PREVENTION OF PRESSURE ULCERS IN NURSING HOMES, A BIG CHALLENGE
}

\author{
Proefschrift \\ ter verkrijging van de graad van doctor aan de Universiteit Maastricht, \\ op gezag van de Rector Magnificus, Prof. dr. Rianne M. Letschert, \\ volgens het besluit van het College van Decanen, \\ in het openbaar te verdedigen \\ op donderdag 13 April 2017 om 16.00
}

door

Martin Willem Franciscus van Leen 


\section{Promotores}

Prof. dr. J.M.G.A. Schols

Prof. dr. S.E.R. Hovius (Erasmus Medisch Centrum Rotterdam)

\section{Co-promotor}

Dr. R.J.G. Halfens

\section{Beoordelingscommissie}

Prof. dr. R.A. de Bie (voorzitter)

Prof. dr. R.R.J.W. van der Hulst

Dr. H.E.W. de Laat (Radboud UMC Nijmegen)

Prof. dr. ir. C.W.J. Oomens (TU Eindhoven)

Dr. A.A.L.M. Rondas 


\section{Contents}

$\begin{array}{lll}\text { Chapter } 1 \text { General introduction } & 7\end{array}$

Chapter 2 Which PRESSURe UlCER PREVENTIVE MeASURes ARE USED IN 23 DUTCH NURSING HOMES? A LONGITUDINAL STUDY FROM 2005-2014

Chapter 3 Pressure Relief, cold foAm or Static Air? A Single Center, 39 PROSPECTIVE, CONTROLLED RANDOMIZED CLINICAL TRIAL IN A DUTCH NURSING HOME

ChAPTER 4 Pressure RELIEF, VisCo-ELASTIC FOAM OR STATIC AIR ON TOP OF 51 IT? A PROSPECTIVE, CROSS-OVER RANDOMIZED CLINICAL TRIAL IN A 7 DUTCH NURSING HOME

ChAPTER 5 THE EFFECT OF A SIMPLE 3-STEP PRESSURE RELIEVING STRATEgy FOR 65 PREVENTION OF PRESSURE ULCERS; AN EXPLORATIVE LONGITUDINAL STUDY FROM 2002-2011

Chapter 6 Preventive efFect of A SKin INTERfaCe Microclimate 81 REGULATING SYSTEM ON PRESSURE ULCER DEVELOPMENT. A MULTICENTER, PROSPECTIVE, CONTROLLED RANDOMIZED CLINICAL TRIAL IN DUTCH NURSING HOMES

Chapter 7 General discussion

ChAPter 8 VALORISATION

SUMMARY 123

SAMENVATTING

DANKWOORD 139

About THe AUthor 145

LIST OF PUBLICATIONS AND PRESENTATIONS 149 
O

6

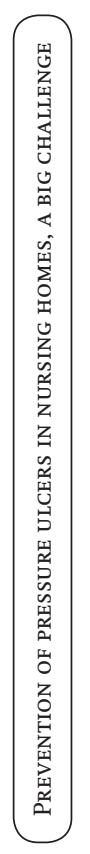




\section{Chapter 1}

GENERAL INTRODUCTION 
O

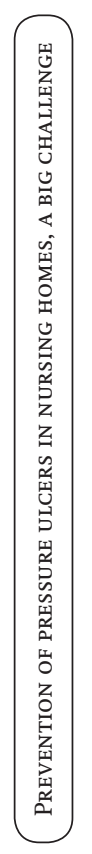




\section{General introduction}

\section{CASE}

A 48-year-old woman with a severe status of multiple sclerosis was staying in our nursing home for several years. She was unable to sit in her wheelchair for a period longer than 2 hours. During the rest of the day she was lying in bed, totally immobile. In bed, she was lying on a low air loss mattress receiving repositioning every 2 hours by the pressure ulcer (PU) protocol. Other $P U$ preventive measures were regular skin assessment, adequate skin care (including use of skin emollients) and taking care of a personalised nutrient and fluid intake. In spite of all the efforts of the nursing staff, she developed a category IV pressure ulcer in the pelvic region. Several interventions (debridement, cleaning, various wound dressings) did not result in complete wound healing. Her condition was not good enough for reconstructive surgery and despite all efforts, complete wound healing was never realised in a period of two years. She stayed in the nursing home, constantly hindered by her PU until she finally passed away.

As shown in this example, PUs can interfere negatively with quality of life in frail and disabled nursing home residents. They cause pain and suffering and can result in social isolation and may also increase morbidity and mortality rates. ${ }^{1}$ Next to this, PUs are associated with extra health care costs, because they need extra intensive specialist nursing and medical care. ${ }^{2}$ Though it is generally accepted that most PUs can be prevented, still too many PUs develop in long-term care facilities like nursing homes.

Although the prevalence of pressure ulcers in Dutch nursing homes has declined during the last 15 years, still $3.2 \%$ of the residents has a PU that has developed in the nursing home itself. ${ }^{3}$ There are less annual (inter)national PU incidence data of long-term care facilities available, but Meesterberends et al. reported an incidence of category II-IV PUs of $21.2 \%$ and $7.8 \%$ in respectively Dutch and German nursing homes, for newly admitted residents that were followed longitudinally for a period of 12 weeks. ${ }^{4}$ De Souza found an incidence of $11.7 \%$ cat II-IV PUs over a period of 90 days in long-term care facilities. ${ }^{5}$ Precise data on the use and type of PU preventive measures in nursing homes are lacking as well and therefore more research is needed on this aspect.

In this thesis, the use of PU preventive measures in nursing homes in the last decade and the effectiveness of some preventive methods, especially mattresses/overlay systems and repositioning are investigated and discussed. 


\section{Definition, staging and aetiology of Pressure Ulcers (PUs)}

\section{Definition and staging}

According to the last National Pressure Ulcer Advisory Panel (NPUAP), European Pressure Ulcer Advisory Panel (EPUAP) and Pan Pacific Pressure Injury Alliance (PPPIA) guideline, PUs are defined as follows: ${ }^{6}$

A pressure ulcer is a localized injury to the skin and/or underlying tissue usually over a bony prominence, as a result of pressure, or pressure in combination with shear.

Pressure ulcers are divided in 6 categories (category I: non-blanchable redness of intact skin; category II: partial thickness skin loss or blister; category III: full thickness skin loss (fat visible); category IV: full thickness tissue loss (muscle/bone visible); and in the last guideline two additional categories have been added: unstageable: depth unknown, and suspected deep tissue injury: depth unknown). ${ }^{6}$ The first four categories are familiar for professionals dealing with pressure ulcers, but because it is not always possible to make the correct diagnosis, two new categories were added in the above mentioned international guideline (i.e. Cat. unstageable and Cat. suspected deep tissue injury). Normally a PU is staged at the moment of noticing a skin lesion. For the two newly added categories, staging is impossible, because the signs of the PU are only clear after debridement or during the healing phase.

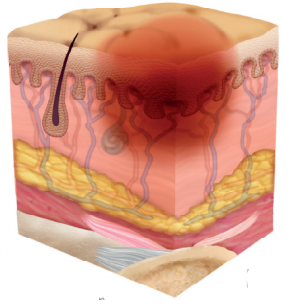

Category 1 Pressure Injury -

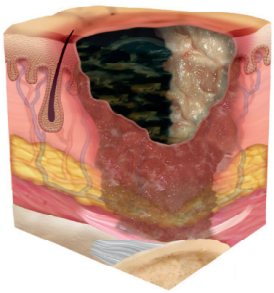

Unstageable Pressure Injury Slough and Eschar

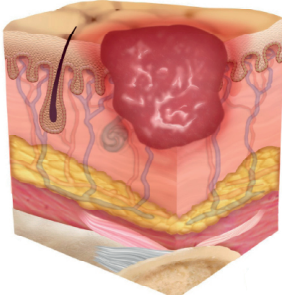

Category 2 Pressure Injury

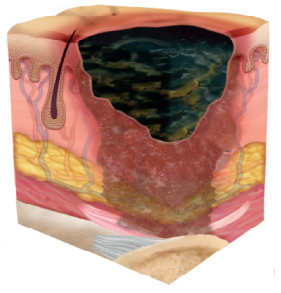

Unstageable Pressure Injury Dark Eschar

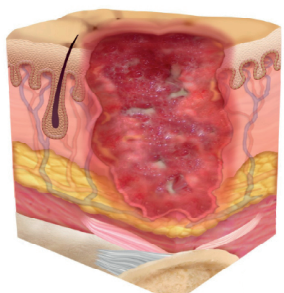

Category 3 Pressure Injury

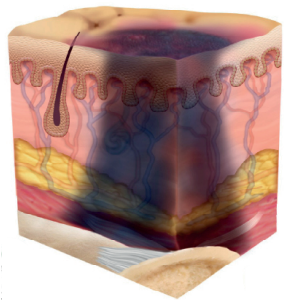

Deep Tissue Pressure Injury

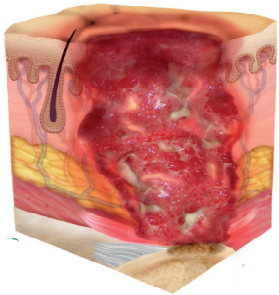

Category 4 Pressure Injury

Figure 1. Categories of pressure ulcers. Used with permission of the National Pressure Ulcer Advisery Panel dd 13 Januari 2017 


\section{Aetiology}

As mentioned in the definition, pressure and shear are important extrinsic factors for the development of PUs. However, pressure and shear do not fully explain the formation of PUs. The effect of pressure and shear is influenced by factors like decreased mobility, neurologic diseases, bad nutritional status, spinal cord lesion, incontinence, bad skin condition or bad tissue viability and microclimate at the skin interface.

Reichel already published in 1958 a conceptual model about the factors 'compressive forces and shearing forces' which could directly contribute to the development of PUs. ${ }^{7}$ Lowthian was in 1970 the first researcher who introduced the effects of tissue tolerance during pressure and shear forces. ${ }^{8}$ Pressure ulcers can start superficially or deep inside the tissue, depending on the nature of the surface loading and tissue integrity. ${ }^{9}$ If a patient cannot move for a protracted length of time, caused by physical restraints or medical conditions, the externally applied pressure may lead to necrosis of the superficial or deep tissue. Based on the latest published theories a difference in causes of development between cat I/II and III/IV ulcers is likely. The several factors which may contribute in the development of a PU will be explained below. ${ }^{10}$

Pressure and shear are the most mentioned direct causes of a pressure ulcer. ${ }^{7,8,9,10}$ Pressure is defined as the amount of force/load applied perpendicular per unit area of application. ${ }^{10} \mathrm{~A}$ force applied over a small area will produce a greater pressure than the same force over a larger area. Two aspects of pressure are important: intensity and duration. The intensity is defined as the amount of external pressure on the skin and the deeper tissue layers. Duration is the time that a certain pressure is exposed to a surface. A short period of high pressure or a longer period of a lower pressure may both result in a PU.

Shear is often used to abbreviate the terms shear stress and shear force. When forces are produced by both a perpendicular component (pressure) and a parallel component to the skin, these are called shear forces. ${ }^{11}$ When dealing with shear forces per unit area the term shear stress is introduced. Shear stress causes tissue deformation.

Friction is no longer mentioned in the description of PU development in the last NPUAP/EPUAP/PPPIA guideline, but there is a close association with shear. Friction is defined as the force that resists the relative motion of two adjacent objects, like skin and support interface. Often friction potentiates shear stress. ${ }^{11}$ 
If pressure and shear are applied to the skin, particularly over a bony prominence, it causes changes of the skin and the underlying tissue. This may result in tissue distortion, compression of blood vessels, stretching and narrowing of the capillary beds, disturbance of the cell metabolism and even cell death. A sufficient high level of shear stresses for a period of 4 hours will result in muscle death. The skin can resist the same type of stress for more than 12 hours. ${ }^{12}$ This is one of the reasons why PUs may already have developed before skin breakdown is visible.

\section{Other Contributing factors}

The development of a PU cannot be fully explained by the effects of pressure and shear stress only. Another important contributing factor also involves the microenvironment/ microclimate, including the skin temperature, moisture and humidity control at the skin-support surface interface (e.g. cotton sheets, silk sheets, mattresses). ${ }^{10,11}$ Already in 1976 Roaf mentioned, that the maintenance of a balanced microclimate was considered to be a key modifier of the ability of the skin and underlying soft tissue to withstand prolonged superficial stress. ${ }^{14}$ However, the question is: what is a good balance? Studies about skin temperature show inconsistent changes of pressure damaged areas. Yusuf et al. found in a study in 2015 that differences in skin temperature seem to be a predictor for PU development. ${ }^{15}$ Nevertheless it is still not certain that a possible dysregulation of skin temperature is related to a higher chance of PU development. Increased skin moisture (incontinence or sweating) is also a possible factor for skin damage by weakening the surface and subcutaneous layer and by increasing the exposure of underlying blood vessels to the effects of pressure and shear stress. Maceration, caused by a higher humidity of the skin, also has negative effects on the strength of the stratum corneum. The higher the humidity, the more vulnerable the tissue. ${ }^{16}$ But there is still no evidence that adequate regulation of skin humidity will prevent development of a pressure ulcer. As Clark et al. concluded the overall aim of microclimate management should be to avoid extremes of temperature or skin moisture. $^{13}$

The NPUAP/ EPUAP/PPPIA guideline also points on other contributing factors like general skin condition, nutritional status, body hydration status and mobility (especially in bed). ${ }^{6}$ Most advices around food intake and nutritional status are based on studies in the elderly. Nutrition is the total process of ingesting nutrients and fluids in sufficient amounts to realise a balanced system, while malnutrition is equal to an imbalanced system. ${ }^{17}$ The diagnosis of malnutrition should be based on a low body mass index (BMI) of $<18.5$ or involuntary weight loss with either an agespecific reduced BMI or a low fat free body mass index and low nutritional intake. 
Inadequate nutritional intake and poor nutritional status have been correlated to the development of PUs. ${ }^{18,19,20,21}$

Sufficient spontaneous mobility, especially in bed, can reduce the risk of development of PUs. ${ }^{22}$ Residents in nursing homes who move spontaneously fewer than 25 times each night were significantly at higher PU risk. Even small, frequent movements like replacement of a leg or arm, may result in reducing the interface pressure over the total body. ${ }^{23,24}$ Stimulation of self-movement should therefore be a standard strategy. The guideline advices for patients, which are not able to replace themselves in bed or in (wheel)chair, are to use repositioning by schedule. ${ }^{6}$

The influence of skin changes during life time on the development of PUs is also a relevant factor. Because of the aging process, a lot of changes occur in skin anatomy and physiology. The epidermis gradually becomes thinner, the dermal-epidermal junction (also called basal membrane) is flattening out and $20 \%$ reduction of the dermis is normal. ${ }^{25,26}$ The flattened interface between epidermis and dermis makes the skin less resistant to shear stress. This process is also one of the reasons, that elderly are more vulnerable for PU development. The complex biochemistry of the dermis also alters because of aging, which results in a decrease of collagen synthesis and progressive loss of elastin making the skin more vulnerable for damage. ${ }^{27}$ Another aspect, related to the aging process, is the reaction of the superficial and deeper vessels during pressure/shear stress and post-loading. Deformation of tissue leads to partial or full compression of the blood vessels, resulting in local ischaemia. Normally after unloading, vessels will rapidly open again and normal blood flow will return. This process, called pressure induced vasodilatation (PIV), is depressed in the elderly. ${ }^{28,29}$ Studies with Doppler flow measurements in combination with a 2-channel photoplethysmography instrument showed different effects on compression in the superficial vessels $(2 \mathrm{~mm})$ and at the level of $10 \mathrm{~mm}$ below skin surface. The same loading resulted in more vessel compression in older people and post-load dilatation occurred more in the superficial vessels and was delayed in the deeper vessels, where sometimes the vessels only opened after a long time. This phenomenon may be important for the choice of pressure redistributing systems. It is possible that the periods between two inflation periods of PU preventive alternating systems are too short to realise full opening of the blood vessels. Loerakker et al. showed that this may result in accumulation of toxic proteases, which may lead to progression of tissue damage. ${ }^{30}$ Further research will be necessary to support this theory. 
Figure 2. Schematic illustration of risk factors that influence pressure ulcer development (partly based on EPUAPNPUAP-PPPIA prevention and treatment of pressure ulcers: clinical practice guideline 2014)

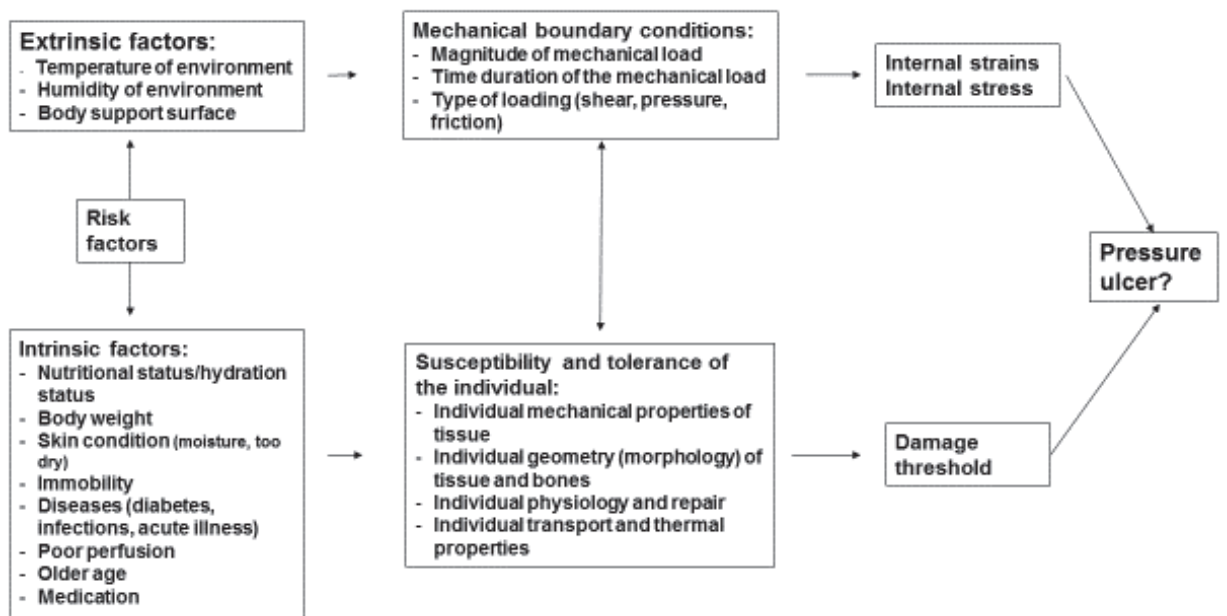

\section{Prevention of pressure ulcers in nursing homes}

In Dutch nursing homes around 65.000 frail, mostly elderly, residents are living, who have a number of diseases, often neurological limitations, mobility disorders and cognitive impairment. ${ }^{3,31} \mathrm{~A}$ high amount of these residents is at medium/high risk for the development of PUs. ${ }^{3}$ Prevention of PU development is therefore of utmost importance and belongs to a good quality of basic care provided by nurses and nurse assistants and the rest of the care team.

The primary aim of pressure ulcer prevention strategies is to reduce either the magnitude and/or duration of pressure between a patient and his (or her) support surface (i.e. the interface pressure), and also reducing shear forces (see fig 2). Reducing the magnitude or duration of pressure may be achieved by using pressure-redistributing support surfaces (such as cushions, mattress overlays, replacement mattresses or whole bed replacements) and/or pressure reduction measures (offloading systems, calf cushions), which are widely used in both institutional and non-institutional settings. Reduction of shear also may be achieved by changes in the materials of the interface layer. Because interference between the skin of the resident and the top layer of the support service can result in negative effects on the microclimate, special attention during selection of the support surface is necessary as well. 
Support surfaces are used with the aim of redistributing pressure, reducing shear/ shear stress forces by immersion (ability to allow a patient to sink in the device) and envelopment (ability to mound to body contours and accommodates irregular areas), while manual repositioning by nurses or the use of alternating systems will lead to shorter periods of high pressure.

The NPUAP/EPUAP/PPPIA guideline advises the standard use of higher-specification foam mattresses for people at high risk of developing pressure ulcers. Furthermore the guideline proposes to consider the use of a high specification reactive foam mattress or non-powered pressure redistribution support surface for individuals with a category I PU. In the Netherlands visco-elastic foam (also called reactive foam or memory foam) mattresses and cold foam mattresses are the mostly used higher-specification foam mattresses. Visco-elastic foam is a polyether foam with an open-cell structure that reacts to body heat and weight by 'molding' to the sleeper's body. Most memory foams have the same basic chemical composition, however differences in the density and layer thickness of the foam makes different mattresses feel very different. A high-density mattress will have better compression ratings over the life of the bedding. A lower-density one will have slightly shorter life due to the compression that takes place after repeated use. The mostly used mattresses have a top layer of visco-elastic foam between 3 and $7 \mathrm{~cm}$ thickness and a cold foam layer below between $8-12 \mathrm{~cm}$. The most important disadvantages of viscoelastic foam are the temperature sensitivity and loss of memory function during life time (most types guaranteed for five years). Cold foam is a poly-urethane foam, but with an open cell structure, which results in good ventilation, is anti-allergic, has no temperature interactions and can absorb a lot of fluid. Lifetime of cold foam mattresses is mostly guaranteed for 10-15 years. Cold foam remodels easily, which results in a better redistribution of pressure. For pressure ulcer prevention, mattresses with specification 50, 55 or $60 \mathrm{HR}$ (high resilience) are advised.

Vanderwee et al. did research on the incidence of PU development on different types of mattresses. They found an incidence of category II, III and IV of 15.3\% during use of a visco-elastic foam mattress and of $15.6 \%$ on alternating air mattresses. ${ }^{32}$

There is no literature available about the preventive value of other types of foam mattresses. The relative merits of higher-specification constant low-pressure mattresses (including static air mattresses) and alternating-pressure support surfaces for preventing pressure ulcers are unclear as well. ${ }^{33}$ 
Therefore, in this thesis several studies about the use of different types of foam mattresses in combination with static air overlays are undertaken in the nursing home setting.

The NPUAP/EPUAP/PPPIA guideline for prevention of PUs also advises to use repositioning for all residents at risk. ${ }^{6}$ However, the evidence to apply repositioning to prevent pressure ulcers is low and we still do not know if particular positions or frequencies of repositioning reduce pressure ulcer development. ${ }^{34}$ As the authors of a Cochrane review (April 2014) advice: further research about the best way of using repositioning is necessary. ${ }^{34}$

Not only the pressure redistributing surface is important; as we have seen, also the humidity between the blankets or mattress cover and the skin of the resident may play an important role in the risk of developing a PU. In a moisture, humid environment the possibility of disruption of the skin barrier function is present, caused by rising of the friction coefficient. Therefore, special attention for the materials in bed (sheets, under layers, lift device) is necessary. ${ }^{11}$ Materials with a high absorption capacity are preferred and materials, which rise the body temperature should be avoided if possible. ${ }^{11}$

Next to the pressure and shear relieving measures and attention for an adequate microclimate, the NPUAP/EPUAP/PPPIA guideline also advises additional PU preventive measures related to the factors that also contribute to PU development. This implies attention for adequate skin care, optimizing disease status and attention for optimal nutritional care. ${ }^{6}$

It will be clear that the recommendations of the guideline have to be implemented adequately in nursing homes to achieve the best pressure ulcer care. Despite this, less is known about the state of implementation in nursing homes of different countries including The Netherlands.

\section{Research questions and outline of the thesis}

In this thesis, first, the state of implementation of PU preventive measures in Dutch nursing homes will be explored. Thereafter, several studies in nursing home residents are undertaken, related to the use of different types of foam mattresses in combination with static air overlays.

The following research questions are addressed: 
1. To what extent did Dutch nursing homes apply PU preventive measures, as advised in valid (inter)national guidelines, during the period 2005-2014?

2. What is the clinical efficacy of combining a standard $15 \mathrm{~cm}$ cold foam mattress with a static air overlay mattress versus a cold foam mattress alone in preventing pressure ulcers in nursing home residents?

3. What is the clinical efficacy of a combination of a standard $15 \mathrm{~cm}$ visco-elastic foam mattress with a static air overlay mattress versus a standard $15 \mathrm{~cm}$ viscoelastic foam mattress alone in preventing pressure ulcers in nursing home residents?

4. What is the effect of implementing a 3 step PU prevention protocol on PU prevalence in the Avoord nursing homes (Etten-Leur, The Netherlands) over the period 2005-2011?

5. What is the effect of a multilayer skin interface microclimate regulating system $\left(\right.$ Bedcare $\left.^{\circledR}\right)$, used in conjunction with a regular, new high quality visco-elastic foam mattress on PU incidence in nursing home residents?

To answer these questions, 5 studies will be performed, using a mixed method strategy. In two studies the methodology of the Dutch national prevalence measurement of care problems - LPZ will be used. In the Netherlands, since 1998, the prevalence of PUs has been measured annually on national level, in different health care sectors (including nursing homes) by the Dutch national prevalence measurement of care problems (LPZ). The LPZ is a cross-sectional, multi-centre point prevalence measurement that uses standardized questionnaires to record next to data about the prevalence and risk of pressure ulcers also data about the preventive and therapeutic measurements taken in daily PU care. The LPZ also measures other relevant care problems like falls, incontinence and malnutrition. The methodology of the LPZ has been described earlier. ${ }^{35}$

In two effect studies with anti PU-mattresses and one effect study on the use of a multilayer skin interface microclimate regulating system, the design of a randomized controlled trial (RCT) will be used. 


\section{Outline of thesis}

The outline of this thesis is as follows:

Chapter 1 involves this general introduction.

Chapter 2 describes a secondary analysis of the use of PU preventive measures in Dutch nursing homes over a period of ten years (2005-2014), by using the data of the annual Dutch national prevalence measurement of care problems - LPZ (see above). In this study, the PU preventive measures as used in Dutch nursing homes during this period are analysed and compared to the during that period valid PU guidelines (EPUAP/NPUAP 1998, 2009 and/or Dutch PU guidelines 2002, 2011).

18 Chapter 3 presents the results of the first randomized controlled trial (RCT), conducted for this thesis. The trial aimed to evaluate the clinical efficacy of combining a standard $15 \mathrm{~cm}$ cold foam mattress with a static air overlay mattress versus a cold foam mattress alone in preventing PUs in nursing home residents.

Chapter 4 reports the results of the second RCT that has been conducted for this thesis. The aim was to evaluate the clinical efficacy of a combination of a standard 15 $\mathrm{cm}$ visco-elastic foam mattress with a static air overlay mattress versus a standard 15 $\mathrm{cm}$ visco-elastic foam mattress alone in preventing PUs in nursing home residents.

Chapter 5 presents the results of the implementation of a three step PU prevention protocol on PU prevalence in the Avoord nursing homes (Etten-Leur/Zundert, The Netherlands) over the period 2005-2011. For this study, the PU prevalence in the Avoord nursing homes in the period 2005-2011 was compared to the national figures of the nursing home sector. In this study also the LPZ data was used, like in chapter 2.

Chapter 6 reports the results of a study on the effect of a multilayer skin interface microclimate regulating system (Bedcare ${ }^{\circledR}$ ), used in conjunction with a regular, new high quality visco-elastic foam mattress on PU incidence in nursing home residents.

Finally, chapter 7 involves the general discussion of the main findings of this thesis. Strengths and limitations of the study methods are discussed and implications for practice and future research are given. 


\section{REFERENCES}

1. Gorecki C, Brown JM, Nelson EA, Briggs M, Schoonhoven L, Dealey C et al. Impact of pressure ulcers on quality of life in older patients: a systematic review. Journal of the American Geriatric Society. 2009;57(7):1175-83

2. Dealy C, Posnett J, Walker A. The cost of pressure ulcers in the United Kingdom. Journal of Woundcare. 2012;21(6):261-6

3. Halfens RJG, Meijers JMM, Meesterberends E, Neyens JCL, Rondas AALM, Rijcken S, Wolters S, Schols JMGA. Landelijke Prevalentiemeting Zorgproblemen Rapportage resultaten 2014 CAPHRI School for Public Health and Primary Care, Department of Health Services Research Focusing on Chronic Care and Ageing. www.lpz-um.eu/media/text/Home/LPZ_Rapport_2014.pdf

4. Meesterberends E, Halfens RJG, Spreeuwenberg MD, Ambergen TAW, Neyens JCL, Schols JMGA. Do residents in Dutch nursing homes have more pressure ulcers than residents in German nursing homes? A prospective multicentre cohort study. JAMDA 2013;14:605-610 doi: 10.1016/j.jamda.2013.03.005.

5. De Souza DM, De Gouveia Santos VL. Incidence of pressure ulcers in the institutionalized elderly. Journal of Wound, Ostomy and Continence Nursing 2010;37:272-276

6. National Pressure Ulcer Advisory Panel, European Pressure Ulcer Advisory Panel and Pan Pacific Pressure Injury Alliance. Prevention and Treatment of Pressure Ulcers: Quick Reference Guide. Emily Haesler (Ed.). Cambridge Media: Perth, Australia; 2014. http://www.npuap.org/wp-content/uploads/2014/08/ Updated-10-16-14-Quick-Reference-Guide-DIGITAL-NPUAP

7. Reichel SM. Shearing force as a factor in decubitus ulcers in paraplegics. J Am Med Assoc; 1958;166(7):762763. doi:10.1001/jama.1958.62990070004010a.

8. Lowthian PT. Bedsores: the missing links? Nursing Times 1970;66:1454-1458

9. Bouten CV, Oomens CW, Baaijens FP \& Bader DL. (2003).The aetiology of pressure ulcers: skin deep or muscle bound? Archives of Physical Medicine \& Rehabilitation 84(4), 616-619.

10. Takahashi M, Black J, Dealey A, Gefen A. Pressure in context. International review. Pressure, shear, friction and microclimate in context. A consensus document. London: Wounds international, 2010

11. Reger SI, Ranganathan VK, Orsted HL, Ohura T, Gefen A. Shear and friction in context. International review. Pressure, shear, friction and microclimate in context. A consensus document. London: Wounds international, 2010

12. Loerakker S. Manders E, Strijkers GJ, Nicolay K, Baaijens FPT, Bader DL, Oomens CWJ. The effects of deformation, ischemia, and reperfusion on the development of muscle damage during prolonged loading. Journal of applied physiology. July 2011 Vol. no.,DOI: 10.1152/japplphysiol.00389.201

13. Clark M, Romanelli M, Reger SI, Ranganathan VK, Black J, Dealey. Microclimate in context. International review. Pressure, shear, friction and microclimate in context. A consensus document. London: Wounds international, 2010

14. Roaf R. The causation of preventing bedsores. J Tissue Viability 2006;16(2):6-8

15. Yusuf S, Okuwa M, Shigeta Y, Dai M, Luchi T, Rahman S, Usman A, Kasim S, Sugama J, Nakatani T, Sanada $\mathrm{H}$. Microclimate and development of pressure ulcers and superficial skin changes. Int Wound J 2015; 12:40-46

16. Brienza DM, Geyer MJ. Using support surfaces to manage tissue integrity. Adv Skin Wound Care 2005;18:151-57 
17. Cederholm T, Bosaeus I, Barazzoni R, et al. Diagnostic criteria for malnutrition- An ESPEN consensus statement. Clinical Nutrition 34 (2015)335-340 http:/dx.doi.org/10.1016/j.clnu.2015.03.001

18. Ek AC, Unosson M, Larsson J, von Schenck H, Bjurulf P. The development and healing of pressure sores related to the nutritional state. Clinical Nutrition. 1991;10(5):245-250

19. Green CJ. Existence, causes and consequences of disease-related malnutrition in the hospital and the community and clinical and financial benefits of nutritional intervention. Clinical Nutrition. 1999;18(suppl.2):3-28

20. Lizaka S, Okuwa M, Kitamura R, Hironori K, Sanada H. The impact of malnutrition and nutrition-related factors on the development and severity of pressure ulcers in older patients receiving home care. Clinical Nutrition. 2010;29(1):47-53

21. Shahin ES, Meijers JM, Schols JMGA, Tannen A, Halfens RJ, Dassen T. The relationship between malnutrition parameters and pressure ulcers in hospitals and nursing homes. Nutrition. 2010 Sep;26(9):886-9.

22. Exton-Smith AN, Sherwin RW. The prevention of pressure sores: significance of spontaneous bodily movements. Lancet 1961;278(7212): 1124-26

23. Brown M, Boosinger J, Black J, Gasper T. Nursing innovation for prevention of decubitus ulcers in longterm care facilities. J Plast Reconstr Surg Nurs 1981; 1(2):51-55

24. Oertwich PA, Kindschuh AM, Bergstrom N. The effects of small shifts in body weight on blood flow and interface pressure. Res Nurs Health 1995; 18(6): 481-488

25. Voegeli, D., Smith \& Nephew Foundation (2007) Factors that exacerbate skin breakdown and ulceration. In, Skin Breakdown: The Silent Epidemic. London, UK, Smith \& Nephew Foundation, 17-21.

26. Voegeli D. Basic essentials: why elderly skin requires special treatment. Nursing \& Residential Care, sept 2010, vol 12, No 9

27. Voegeli D. Age related changes in the skin. Dermalogical nursing.2003,2(4), 5-6

28. McLellan K, Petrofsky JS, Zimmerman G, Lee S. Multiple stressors and the response of vascular endothelial cells: the effect of aging and diabetes. Diabetes Technol Ther. 2009 Feb;11(2):73-9. doi: 10.1089/dia.2008.0026

29. Bergstrand S, Lindberg LG, Ek AC, Lindgren M. Blood flow measurements at different depths using photoplethysmography and laser doppler techniques. Skin Reasearch and Technology, 2009,(15),2,139147

30. Loerakker S, Manders E, Strijkers GJ, Nicolay K, Baaijens FP, Bader DL, Oomens CW. The effects of deformation, ischemia, and reperfusion on the development of muscle damage during prolonged loading. J Appl Physiol (1985). 2011 Oct;111(4):1168-77. doi: 10.1152/japplphysiol.00389.2011. Epub 2011 Jul 14

31. Centraal Plan Bureau. Zorg in de laatste jaren (2014). https://www.scp.nl/dsresource?objectid=936ed727af5c-42cd

32. Vanderwee K, Grypdonck MH, Defloor T. Effectiveness of an alternating pressure air mattress for the prevention of pressure ulcers. Age Ageing. 2005 May;34(3):261-7. Epub 2005 Mar 11.

33. McInnes E, Jammali-Blasi A, Bell-Syer SEM, Dumville JC, Middleton V, Cullum N. Support surfaces for pressure ulcer prevention. Cochrane Database of Systematic Reviews 2015, Issue 9. Art. No.: CD001735. DOI: 10.1002/14651858.CD001735.pub5. 
34. Gillespie BM, Chaboyer WP, McInnes E, Kent B, Whitty JA, Thalib L. Repositioning for pressure ulcer prevention in adults. Cochrane Database of systematic reviews 2014. DOI: 10.1002/14651858.CD009958. pub2

35. Van Nie-Visser NC, Schols JMGA, Meesterberends E, Lohrmann C, Meijers JMM, Halfens RJG. An International prevalence measurement of care problems: study protocol. Journal of Advanced Nursing. Volume 69, Issue 9, 18-29,September 2013. DOI: 10.1111/jan.12190 
O

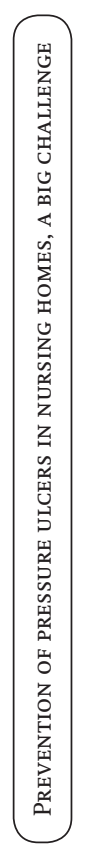


CHAPTER 2

\section{WHICH PRESSURE ULCER PREVENTIVE}

MEASURES ARE USED IN DUTCH NURSING

HOMES? A LONGITUDINAL STUDY FROM 2005-

2014

van Leen MWF, Schols JMGA, Hovius SER, Halfens RJG. 


\section{Abstract}

\section{Objective:}

Pressure ulcers (PUs) remain an important and distressing problem in (Dutch) nursing homes. Prevention is still better than healing pressure ulcers. Therefore we analyzed which preventive measures are used over the years in Dutch nursing homes in relation to the running guidelines.

\section{Method:}

Over the period 2005-2014 data about the use of recommended preventive measures

24 for residents at risk of PUs in Dutch nursing homes were collected. Three groups were distinguished: 1) residents with a Braden score of 17, 18 and 19;2) residents with a Braden score of less than 17; and 3) residents with a pressure ulcer. Data were derived from the annual independent Dutch National Prevalence Measurement of Care Problems (LPZ), a multi-centre cross-sectional point-prevalence measurement of Maastricht University, the Netherlands.

\section{Results:}

The use of preventive measures was higher when the risk of PU development was higher or when PUs were already present. However, in all three groups none of the analysed recommended PU preventive measures was used as advised in the guidelines.

Over the years, in residents of the three groups skin assessment was performed between 25 and 60\%; attention for malnutrition and dehydration varied between 28 and 65\%; use of repositioning between 3 and 52\%; use of floating heels varied from 8 to $58 \%$; use of pressure redistributing systems from 59 to $85 \%$; and application of pressure relieving cushions from 21 to $65 \%$.

\section{Conclusion:}

None of the advised preventive measures of both the guidelines of EPUAP/NPUAP 1998 and 2009 and the Dutch PU guidelines of 2002 and 2011, were fully applied in the time frame of this study. Why the preventive measures were not fully used, is unclear. Possible explanations may be a lack of awareness and knowledge, the attitude of the nursing staff and the fact that the guideline recommendations might be insufficiently tailored to daily practice of PU care. 


\section{Introduction}

Pressure ulcers (PUs) are an important and distressing care problem in nursing homes, leading to real suffering and loss of quality of life of the residents involved. ${ }^{1}$ Next to this, PUs are associated with considerable extra health care costs. ${ }^{2}$

Use of evidence and/or practice based preventive measures may result in a lower incidence of PUs. ${ }^{3,4,5}$ The right application of PU preventive measures can be facilitated by implementing the recommendations of evidence based (inter)national guidelines.

In this study, the preventive measures used in Dutch nursing homes, in the period 2005-2014, are investigated and compared to the then valid guidelines (EPUAP/ NPUAP1998 and 2009 and Dutch guidelines 2002 and 2011).

A pressure ulcer is a localized injury to the skin and/or underlying tissue, usually over a bony prominence that results from pressure, including pressure associated with shear. ${ }^{3,4,5,6}$

Pressure has long been viewed as the most important extrinsic factor of pressure ulcer development. In addition, shear, shear stress and the microclimate at the skin interface also play a very important role in the pathophysiology.? Several theories about the development of PUs circulate. The oldest theory implies a gradual development of damage from the superficial skin layer to the deep soft tissues. $^{8}$

Another theory implies that ulcer damage originates primarily in the deeper tissues and then develops further throughout the more superficial layers towards the skin. ${ }^{9}$

Nowadays, PUs are often divided into shear stress ulcers that develop at the surface, primarily caused by friction, shear and trauma (category 1 and 2) and ulcers starting in deep tissue, mainly caused by perpendicular forces and shear strength (category 3 and 4$){ }^{9}$

The significance of friction in causing PUs lies mainly in its contribution to the production of shear stress. When the friction related tangential force is higher at the skin surface than the perpendicular force (pressure), or when a small amount of pressure with a large tangential force is applied to the skin, abrasions, superficial ulceration or blistering of the skin may occur. ${ }^{9}$ 
In addition to these direct causing factors, a number of other, more indirect factors have been associated with the occurrence of pressure ulcers. These include mobility problems; neurological diseases; reperfusion; (re)perfusion/oxygenation damage; skin moistness; skin/body temperature; general health; age and nutritional status. ${ }^{3,4,5,6}$ Based on both these direct and indirect causal factors, the appropriate preventive measures should be chosen.

In the last decade an integral approach regarding the application of preventive measures in PU care is advised, consisting of: PU risk assessment, skin assessment, assessment and optimization of nutritional status, pressure relief/redistribution and repositioning. ${ }^{3,4,5,6} \quad$ The guidelines do not give clear directions under which circumstances some or all measures should be used.

PU risk assessment, as advised by the (inter)national guidelines, can be done with a structured instrument like the Braden scale, Waterloo scale or Norton scale and/or by a clinical assessment (daily skin control). ${ }^{10,11,12}$

In the Netherlands, the Braden scale is the mostly accepted one and also used in the Dutch prevalence studies. ${ }^{10,13}$ The scale consists of the following items: sensory perception, moisture, activity, nutritional intake, and friction and shear, with scores ranging from 1 to 3 or 4 points. The maximum score is 23 points (no risk at all). A score lower than 20 indicates that residents are at risk of a PU. A score less than 17 indicates being at medium/high risk of a PU. ${ }^{13}$

A proper skin assessment and skin care involve daily control of the skin searching for redness, localized heat, edema or induration and paying attention to an adequately hydrated skin. ${ }^{3,4,5,6}$

Since undernutrition is a reversible risk factor, which influences pressure ulcer development, early identification and management of nutritional problems is very important. ${ }^{3,4,5,6}$ Especially, because it is well known that many PU-patients have a compromised nutritional status. ${ }^{14,15}$

The most important preventive measure is of course pressure relief and shear prevention, because this action interferes with the basic reasons for PU development. In all relevant guidelines, as a first step, the use of pressure relieving systems in bed and (wheel)chair is advised. Pressure redistributing systems, therefore, play an important role in the daily practice of pressure ulcer prevention. Physicians and nurses often apply additional support surfaces to redistribute pressure over a larger 
surface area of the patient's body. ${ }^{3,4,5,6} \mathrm{~A}$ distinction can be made between passive and active systems. A passive system involves the use of a higher specification foam mattress than the usual standard hospital foam mattress. An active system (alternative system) combines redistribution with varying pressure levels in time. Use of an alternating mattress (active system) is indicated when a visco-elastic foam mattress is not realizing adequate prevention.

It is recommended to combine a higher specification visco-elastic foam mattress or an alternating mattress with repositioning. ${ }^{3,4,5,6}$ Repositioning is advised every 3 hours during the day and every four hours during the night for individuals at risk, unless contra-indicated. ${ }^{3,4,5,6}$

The aim off this study was to explore which PU preventive measures were used in Dutch nursing homes over the period 2005-2014 in relation to the running guidelines.

\section{Method/Design of the study}

A secondary analysis was performed on data from the Dutch National Prevalence measurement of Care problems (Landelijke Prevalentiemeting Zorgproblemen (LPZ). Since 1998, Maastricht University conducts an annual 1-day national prevalence measurement (LPZ) of relevant care problems, such as pressure ulcers, malnutrition and incontinence in Dutch hospitals, nursing homes and home care organizations. The LPZ is a cross-sectional, multi-centre point prevalence measurement that uses standardized questionnaires to record data about the prevalence and risk of pressure ulcers and also data about the preventive and therapeutic measures taken in daily PU care. ${ }^{16}$ Participation in the LPZ enables nursing homes to compare their PU prevalence, PU prevention policy and PU treatment policy annually with national data from the total nursing home sector in the country.

For this study, focusing on nursing homes, data were used from the LPZ measurements from 2005 to 2014.

\section{Participants}

Yearly all Dutch nursing homes are invited to participate. To obtain representative results, nursing homes are encouraged to measure the residents of all wards. Residents who receive palliative care, short-term rehabilitation or who stay in the nursing home for less than 30 days are excluded by protocol. For this study, the following at risk groups were distinguished and selected: 
1. Residents at low risk of a PU (Braden score17-19)

2. Residents at medium/high risk of PUs (Braden score<17)

3. Residents with one or more PUs category 2 or higher

\section{Instruments}

Besides relevant demographic data and data on the care dependency of the residents also data related to the risk of pressure ulcers, the classification of PUs, and the preventive measures used for preventing PUs were used in this secondary analysis.

Care dependency was measured with the Care Dependency Scale. ${ }^{17}$ This instrument exists of the following items, each with a 5-point Likert scale (from completely agree to completely disagree): eating/drinking, continence, body posture, mobility, day/night pattern, getting dressed/undressed, body temperature, hygiene, avoiding of danger, communication, contact with others, sense of rules and values, daily activity, recreational activities and learning ability. The total score reaches from 15 to 75 points. The higher the score, the less care dependent the resident is.

The risk of pressure ulcers was measured with the Braden scale (Braden, 1987). ${ }^{10,13}$ PUs were classified according to the pressure ulcer grading system of the EPUAP (first years EPUAP 1998, last year's EPUAP/NPUAP 2009). ${ }^{6,8}$

Related to the guidelines the following recommended preventive measures were measured in the LPZ: structured skin assessment/care (including use of protecting crèmes), identification and management of dehydration and/or malnutrition (including BMI, attention for nutritional intake, supplementation of deficiencies), reposition pattern (repositioning by time schedule in bed and chair), use of floating heels (offloading or use of heel devices) and use of pressure redistributing systems (type of mattresses and cushions).

The applied preventive measures were derived from the resident files.

\section{Statistical analyses}

Data were analyzed using the IBM Statistical Package for the Social Sciences, SPSS, version 23.0.

Three files were made from the total data file of the years 2005 to 2014 , based on the at risk degree of the residents: 1 . Residents at low risk of pressure ulcers (Braden score 17,18 or 19$)$ without a pressure ulcer; 2 . Residents at medium/high risk of 
pressure ulcers (Braden score less than 17) without a pressure ulcer; and 3. Residents with a pressure ulcer category 2 and higher.

Next, each file was aggregated on institutional level, due to the fact that the prevalence rate is a primary characteristic of the nursing home.

The mean characteristics of the residents, the mean use of preventive measures, and the mean use of preventive mattresses on institutional level were calculated and differences between the years were tested with regression analyses, using 'year' as independent variable.

\section{Results}

In table 1 the main characteristics of the study population over the years are shown.

Table 1. Mean resident characteristics of all residents at risk, aggregated on institutional level (2005-2014)

\begin{tabular}{|l|c|c|c|c|c|c|c|c|c|c|c|}
\hline Year of measurement & $\mathbf{2 0 0 5}$ & $\mathbf{2 0 0 6}$ & $\mathbf{2 0 0 7}$ & $\mathbf{2 0 0 8}$ & $\mathbf{2 0 0 9}$ & $\mathbf{2 0 1 0}$ & $\mathbf{2 0 1 1}$ & $\mathbf{2 0 1 2}$ & $\mathbf{2 0 1 3}$ & $\mathbf{2 0 1 4}$ & p-value \\
\hline Number of residents & 6400 & 7392 & 11096 & 6756 & 7345 & 6165 & 3710 & 2442 & 1493 & 1553 \\
\hline Number of institutions & 90 & 108 & 148 & 92 & 175 & 163 & 89 & 64 & 39 & 38 \\
\hline Mean age & 82.4 & 82.6 & 82.2 & 81.9 & 83.6 & 83.4 & 83.8 & 83.9 & 82.8 & 81.8 & $\mathrm{p}<.01$ \\
\hline Mean \% females & 73.3 & 74.9 & 73.4 & 73.3 & 76.8 & 76.8 & 74.1 & 72.6 & 69.8 & 70.5 & $\mathrm{~ns}$ \\
\hline Mean Braden Score & 15.2 & 15.0 & 15.0 & 14.9 & 15.4 & 15.6 & 15.4 & 15.4 & 15.5 & 15.7 & $\mathrm{p}<.0001$ \\
\hline Mean Care Dependency & $*$ & $*$ & 33.3 & 31.6 & 34.3 & 33.1 & 31.8 & 32.2 & 33.0 & 35.0 & $\mathrm{~ns}$ \\
\hline $\begin{array}{l}\text { Mean \% pressure ulcer } \\
\text { (cat. 2, 3 and 4) }\end{array}$ & 16.6 & 15.3 & 10.7 & 9.8 & 7.1 & 7.0 & 6.1 & 7.2 & 4.0 & 5.5 & $\mathrm{p}<.0001$ \\
\hline
\end{tabular}

* $=$ not measured

During the period 2005-2014, the mean age and mean Braden score differ significantly between the years. However the differences are very small. No significant difference exists regarding gender and care dependency.

Although these demographic characteristics don't show large differences between the years, the PU prevalence shows an almost linear decrease during the first years (2005-2009) and stabilization in the period 2010-2014.

In table 2 the results of four recommended measures for PU prevention are presented for each at risk group. As it could be expected, the use of these measures increases with an increase of the PU risk. 
Table 2. Use of preventive measures per year and group (\%) aggregated on institutional level (2005-2014)

\begin{tabular}{|c|c|c|c|c|c|c|c|c|c|c|c|}
\hline Year of measure & 2005 & 2006 & 2007 & 2008 & 2009 & 2010 & 2011 & 2012 & 2013 & 2014 & p-value \\
\hline Number of residents group 1 & 1970 & 2225 & 3466 & 2018 & 2519 & 2229 & 1315 & 907 & 553 & 646 & \\
\hline Number of residents group 2 & 3507 & 4075 & 6350 & 4052 & 4183 & 3488 & 2134 & 1386 & 862 & 802 & \\
\hline Number of residents group 3 & 903 & 1060 & 1220 & 662 & 614 & 428 & 242 & 139 & 68 & 95 & \\
\hline \multicolumn{12}{|l|}{ Skin assessment / Skin care } \\
\hline Group 1 & 25.1 & 23.7 & 31.1 & 36.8 & 28.9 & 29.1 & 35.3 & 35.6 & 40.4 & 38.5 & $\mathrm{p}<.0001$ \\
\hline Group 2 & 35.0 & 32.6 & 44.1 & 49.8 & 49.5 & 42.9 & 54.4 & 53.6 & 56.7 & 52.2 & $\mathrm{p}<.0001$ \\
\hline Group 3 & 34.6 & 36.4 & 50.5 & 60.9 & 55.0 & 52.4 & 54.1 & 55.4 & 63.8 & 60.6 & $\mathrm{p}<.0001$ \\
\hline \multicolumn{12}{|c|}{ Identification and management of dehydration and/or malnutrition } \\
\hline Group 1 & 30.1 & 34.3 & 29.5 & 28.0 & 30.0 & 27.8 & 31.2 & 36.7 & 39.0 & 36.1 & ns \\
\hline Group 2 & 36.4 & 41.7 & 42.4 & 41.6 & 43.8 & 42.2 & 43.6 & 45.5 & 51.1 & 46.2 & ns \\
\hline Group 3 & 39.3 & 47.2 & 50.6 & 51.5 & 54.6 & 55.3 & 47.7 & 58.8 & 65.6 & 58.6 & $\mathrm{p}<.0001$ \\
\hline \multicolumn{12}{|l|}{ Reposition pattern } \\
\hline Group 1 & 6.5 & 9.7 & 5.6 & 5.7 & 3.8 & 5.2 & 3.3 & 4.9 & 2.8 & 6.7 & $p<.01$ \\
\hline Group 2 & 29.4 & 32.4 & 30.3 & 27.4 & 25.8 & 27.5 & 25.8 & 22.8 & 21.4 & 18.4 & $\mathrm{p}<.0001$ \\
\hline Group 3 & 38.9 & 41.5 & 42.7 & 48.7 & 48.3 & 42.8 & 44.9 & 36.5 & 51.6 & 42.5 & ns \\
\hline \multicolumn{12}{|c|}{ Use of floating heels or heel devices } \\
\hline Group 1 & 7.9 & 11.4 & 9.3 & 9.9 & 8.2 & 8.7 & 10.0 & 9.4 & 11.3 & 9.4 & ns \\
\hline Group 2 & 20.3 & 22.6 & 23.9 & 25.4 & 26.3 & 25.0 & 27.2 & 28.2 & 31.0 & 30.5 & $\mathrm{p}<.0001$ \\
\hline Group 3 & 34.0 & 38.1 & 46.7 & 47.0 & 53.5 & 49.4 & 50.5 & 51.7 & 57.8 & 52.8 & $\mathrm{p}<.0001$ \\
\hline
\end{tabular}

Group 1: residents with low risk (Braden score 17-19), without pressure ulcers

Group 2: residents with medium/high risk (Braden score 16 and lower), without pressure ulcers

Group 3: residents with one or more pressure ulcers

\section{Skin assessment / Skin care}

Over the years, in all three groups a statistical difference is found in paying special attention to skin condition.

Although the attention for structured skin assessment increases during the years, in 2014 still about $60 \%$ of the residents in the low risk group, $45 \%$ in the medium/high risk group and $40 \%$ in the group with PUs did not receive special attention related to the skin condition.

\section{Identification and management of dehydration and/or malnutrition}

Although identification and management of dehydration and/or malnutrition differs between the years in all three at risk groups, only the group of residents with a PU shows a significant increase in these preventive measures over the studied years.

Yet, in all groups many residents did not get adequate attention for possible dehydration and/or malnutrition. 


\section{Reposition pattern}

The use of repositioning differs between the years, which makes it difficult to draw conclusions. Only during the last years a significant decline of repositioning occurs in the at risk group with a Braden score of less than 17. In 2014 only 18.4\% of the residents of this group received repositioning by time schedule.

\section{Use of floating heels or heel devices}

The use of floating heels or heel devices shows an increase during the years in both the group with a medium/high risk of PUs and the group with a PU. In the group of residents at low risk, the use of these offloading devices remains very low during the years.

\section{Pressure redistributing systems}

Table 3 shows the use of pressure redistributing systems in bed. The table shows that during the years no significant differences are found regarding the total number of pressure redistributing mattresses/overlays. However, pressure redistributing systems were used more often when the PU risk is higher.

Table 3. Pressure redistributing systems per year and nursing home resident group (\%) aggregated on institutional level (2005-2014)

\begin{tabular}{|c|c|c|c|c|c|c|c|c|c|c|c|}
\hline Year of measure & 2005 & 2006 & 2007 & 2008 & 2009 & 2010 & 2011 & 2012 & 2013 & 2014 & p-value \\
\hline \multicolumn{12}{|l|}{ Mattresses } \\
\hline \multicolumn{12}{|c|}{ Alternating air mattress } \\
\hline Group 1 & 3.9 & 6.1 & 4.7 & 4.8 & 4.3 & 4.4 & 5.5 & 4.3 & 1.2 & 1.2 & ns \\
\hline Group 2 & 11.5 & 15.5 & 12.2 & 12.5 & 11.6 & 13.2 & 13.5 & 12.7 & 6.0 & 5.1 & $\mathrm{p}=.02$ \\
\hline Group 3 & 21.5 & 28.0 & 26.4 & 29.7 & 24.9 & 31.8 & 31.8 & 25.6 & 17.0 & 14.7 & ns \\
\hline \multicolumn{12}{|c|}{ Air (overlay) mattress } \\
\hline Group 1 & 6.2 & 5.1 & 5.2 & 6.8 & 5.8 & 6.3 & 5.2 & 4.5 & 16.0 & 13.5 & $\mathrm{p}<.01$ \\
\hline Group 2 & 9.2 & 10.8 & 11.9 & 13.8 & 13.7 & 11.1 & 11.2 & 9.4 & 25.9 & 23.1 & $\mathrm{p}<.0001$ \\
\hline Group 3 & 13.8 & 18.7 & 19.6 & 21.9 & 29.4 & 18.5 & 18.6 & 18.2 & 44.9 & 39.0 & $p<.0001$ \\
\hline \multicolumn{12}{|l|}{ Foam mattress } \\
\hline Group 1 & 63.8 & 56.2 & 54.8 & 54.7 & 47.4 & 47.7 & 59.4 & 57.2 & 54.6 & 54.3 & ns \\
\hline Group 2 & 53.2 & 47.9 & 46.0 & 47.2 & 40.7 & 39.5 & 49.7 & 48.1 & 43.4 & 45.7 & ns \\
\hline Group 3 & 37.3 & 32.5 & 32.4 & 30.0 & 21.6 & 26.5 & 34.8 & 37.0 & 9.6 & 23.5 & ns \\
\hline \multicolumn{12}{|c|}{ Total of use pressure redistributing mattresses } \\
\hline Group 1 & 73.9 & 67.4 & 64.7 & 66.3 & 57.5 & 58.4 & 70.1 & 66.0 & 71.8 & 69.0 & ns \\
\hline Group 2 & 73.9 & 74.2 & 70.1 & 73.5 & 66.0 & 63.8 & 74.4 & 70.2 & 75.3 & 73.9 & ns \\
\hline Group 3 & 72.6 & 79.2 & 78.4 & 81.6 & 75.9 & 76.8 & 85.2 & 80.8 & 71.5 & 77.2 & ns \\
\hline
\end{tabular}

Group 1: residents with low risk (Braden score 17-19), without pressure ulcers

Group 2: residents with medium/high risk (Braden score 16 and lower), without pressure ulcers

Group 3: residents with one or more pressure ulcers 
Since 2012, the results show a decline in the use of alternating mattresses in all groups in favor of the use of static air overlay mattresses.

The use of foam mattresses in general is stable over the years, but these mattresses are used less when the PU risk is higher.

\section{Pressure redistributing cushions}

The total use of pressure redistributing cushions is rather stable during the years. Since 2013 the use seems to be growing in all three groups (table 4). This is caused mainly by an increase of the use of air cushions. The use of a foam cushion declines, especially in the low and medium/high risk group.

Table 4. Pressure redistributing cushions in (wheel)chairs per year and group (\%) aggregated on institutional level (2005-2014)

\begin{tabular}{|c|c|c|c|c|c|c|c|c|c|c|c|}
\hline Year of measure & 2005 & 2006 & 2007 & 2008 & 2009 & 2010 & 2011 & 2012 & 2013 & 2014 & p-value \\
\hline \multicolumn{12}{|c|}{ Cushions in (wheel)chairs } \\
\hline \multicolumn{12}{|l|}{ Gel pad } \\
\hline Group 1 & 7.5 & 6.1 & 5.0 & 6.6 & 5.8 & 4.8 & 5.4 & 4.1 & 0.9 & 3.1 & $p<.01$ \\
\hline Group 2 & 7.7 & 7.7 & 7.2 & 9.0 & 9.4 & 8.3 & 8.3 & 5.6 & 3.8 & 5.0 & ns \\
\hline Group 3 & 9.3 & 6.7 & 8.2 & 10.0 & 9.6 & 13.3 & 7.3 & 3.1 & 6.3 & 8.3 & ns \\
\hline \multicolumn{12}{|l|}{ Air cushion } \\
\hline Group 1 & 7.2 & 7.9 & 7.6 & 9.8 & 5.9 & 4.8 & 8.0 & 5.7 & 21.4 & 20.9 & $\mathrm{p}<.0001$ \\
\hline Group 2 & 8.6 & 9.7 & 11.3 & 12.9 & 8.0 & 7.5 & 11.9 & 10.4 & 31.5 & 27.9 & $\mathrm{p}<.0001$ \\
\hline Group 3 & 13.7 & 12.9 & 15.6 & 18.2 & 11.8 & 9.5 & 13.4 & 16.7 & 50.0 & 33.5 & $\mathrm{p}<.001$ \\
\hline \multicolumn{12}{|l|}{ Foam cushion } \\
\hline Group 1 & 23.3 & 17.7 & 17.4 & 17.4 & 12.0 & 10.6 & 11.0 & 11.0 & 9.2 & 9.4 & $p<.0001$ \\
\hline Group 2 & 24.6 & 19.7 & 20.1 & 20.7 & 17.0 & 15.7 & 14.7 & 15.8 & 12.6 & 10.9 & $\mathrm{p}<.0001$ \\
\hline Group 3 & 17.7 & 17.9 & 20.5 & 18.4 & 14.7 & 16.7 & 15.4 & 10.8 & 8.5 & 18.7 & ns \\
\hline \multicolumn{12}{|c|}{ Total of pressure redistributing cushions } \\
\hline Group 1 & 38.0 & 31.7 & 30.0 & 33.8 & 23.7 & 20.2 & 24.4 & 20.8 & 31.5 & 33.4 & $\mathrm{p}<.0001$ \\
\hline Group 2 & 40.9 & 37.1 & 38.6 & 42.6 & 34.4 & 31.5 & 34.9 & 31.8 & 47.9 & 43.8 & ns \\
\hline Group 3 & 40.7 & 37.5 & 44.3 & 46.6 & 36.1 & 39.5 & 36.3 & 30.8 & 64.8 & 60.5 & ns \\
\hline
\end{tabular}

Group 1: residents with low risk (Braden score 17-19), without pressure ulcers

Group 2: residents with medium/high risk (Braden score 16 and lower), without pressure ulcers

Group 3: residents with one or more pressure ulcers

\section{Discussion}

This study shows that the overall use of PU preventive measures is higher when the risk of PU development is higher or when PUs are already present. However, not all at risk residents receive the recommended PU preventive measures as advised in 
the guidelines, such as skin assessment or a pressure redistributing matress .,4,5,6 $^{3,6}$ Over the years, the data show an increase of the use of adequate skin care, the use of static air overlays and preventive air cushions in all three groups, and also an increase in the use of offloading heel devices in group two and three. A decrease was found in repositioning and the use of alternating mattressess in group two, and also in the use of foam cushions in group one and two. Overall, the use of pressure relieving systems was stable.

The application of adequate skin care showed a statistically significant increase over the years for all groups, but never reached a percentage higher than $60 \%$. Because adequate skin care is very important, a $100 \%$ use was expected. Attention for malnutrition and dehydration only showed a statistically significant increase in the group of residents with a PU, but it never reached a higher percentage than $65.6 \%$. This fits in the results of a study in Austrian nursing homes, revealing that attention for nutritional status was only present in $53.5 \%$ of the residents at risk of a PU. ${ }^{18}$

Repositioning is an integral component of pressure ulcer prevention and treatment and is widely recommended. Remarkably, this study shows that it is not widely used in daily practice. ${ }^{19}$

Moore et al. (2011) found a reposition pattern of 9\% in older residents of Irish long term care facilities. ${ }^{20}$ Contrary to our data, they found no difference in the use between residents with and without a PU. Halfens et al. (2013) found that about $40 \%$ of the residents at risk of a PU in Austrian nursing homes received repositioning.

For heel offloading, in this study a maximum of 55\% (in the group of residents with a PU) was found, while in Austrian nursing homes over $60 \%$ of the residents at risk received offloading. ${ }^{19}$ Pressure redistributing mattresses were used in $70 \%$ of the residents in all our groups and this percentage increased with about $10 \%$ after development of a PU, while in nursing homes in Ireland 50\% of the residents had a pressure redistribution device in bed and in Austrian nursing homes more than $80 \%$ of the residents were lying on a pressure redistributing device.

\section{Possible reasons for insufficient implementation of recommended measures}

The main question that remains is why the PU preventive measures are not fully used as advised in the guidelines. Lack of awareness and unfamiliarity with the recommended measures might be important reasons why nurses do not act according to the guidelines. 
In general, it is a known phenomenon that the implementation of (inter)national guidelines in daily practice often is insufficient. ${ }^{21,22,23}$

Therefore, the aspects of knowledge and attitude should receive more intention. Meesterberends et al. (2010) indicated that in Dutch nursing homes most nurses were not aware of the guideline recommendations regarding PU prevention. ${ }^{24}$ Demarré et al. found in a study in Belgian elderly care facilities that the knowledge of nurses about PU preventive measures was low and that the attitude of the nursing staff was a predictor of the application of fully compliant prevention for residents at risk of PUs. ${ }^{25}$

Because this study reveals that the implementation of evidence based PU preventive measures remains a challenge, the question also arises whether the current recommendations of the guidelines are tailored enough to the daily practice of PU care. PU guidelines should explain better which (combinations of) preventive measures should be used in specific resident situations to offer the possibility to tailor the application of PU preventive measures more to the individual resident and thereby facilitating their application in daily resident care.

Additionally, the implementation of guidelines might be facilitated by easy to use tools such as checklists, alerts and perhaps also by innovative and feasible apps. Finally adequate coaching on the job should be provided, instead of only providing hard to read, massive guideline documents.

This study of course had some limitations. Table 1 (general characteristics) shows that during the period 2005-2014 the mean age and mean Braden score differed significantly between the years. However these differences were very small. Due to the large total number of participants, statistical significance is reached already for small differences. However, whether such small differences are relevant for general practice is unclear. Because the methodology of the LPZ measurement involves a cross-sectional design, it is impossible to make causal conclusions. In addition, though most of the participating nursing homes are involved annually in this national measurement, not every nursing home participates every year.

Nevertheless, despite these limitations, the data are rather stable over the years.

This study was performed without any conflicts of interest. All authors made a substancial contribution to the article. 


\section{REFERENCES}

1. Gorecki C, Brown JM, Nelson EA, Briggs M, Schoonhoven L, Dealey C et al. Impact of pressure ulcers on quality of life in older patients: a systematic review. Journal of the American Geriatric Society. 2009;57(7):1175-832.

2. Dealy C, Posnett J, Walker A. The cost of pressure ulcers in the United Kingdom. Journal of Woundcare. 2012;21(6):261-6

3. NPUAP/EPUAP. Pressure Ulcer Prevention quick reference guide 2009. http://www.npuap.org/wp-content/ uploads/2012/02/Final_Quick_Prevention_for_web_2010.pdf

4. V\&VN. Dutch national guidelines for prevention and treatment of pressure ulcers. 2011 http://www. venvn.nl/LinkClick.aspx?fileticket=8TrtENI2ZjE\%3D\&tabid=1852.

5. EPUAP guidelines prevention and treatment 1998. www.epuap.com.

6. Prevention and treatment of pressure ulcers, a Dutch guideline, CBO (Dutch institute for healthcare improvement), Utrecht 2002.

7. MacGregor L, Orsted H, Ohura T, Harding $\mathrm{K}$ et al. International Review: Pressure ulcer prevention: pressure, shear, friction and microclimate in context, a consensus document. Wounds international 2010.

8. Orsted HL, Ohura T, Harding K. International review. Pressure ulcer prevention: Pressure, shear, friction and microclimate in context. A consensus document Londen Wounds International, 2010

9. Reger SI, Ranganathan VK, Orsted HL, Ohura T, Gefen A. Shear and friction in context. International review. Pressure ulcer prevention: pressure, shear, friction and microclimate in context. A consensus document Londen Wounds International, 2010.

10. Braden D. Calculating the risk: reflections on the Braden Scale. Advances in Wound Care 1989;9:38-43.

11. Norton D, Exton-Smith AN, McLaren R. An investigation of geriatric nursing problems in hospital. London: National corporation for the care of old people; 1962

12. Waterlow JA. A risk assessment card. Nurs times 1985;81(49):5155

13. Halfens RJG, van Achterberg T, Bal RM. Validity and reliability of the Braden scale and the influence of other risk factors: a multi-center prospective study. International Journal of Nursing Studies 37 (2000), 313-319.

14. Meijers JMM, Schols JMGA, Van Bokhorst-van der Schueren MAE, Dassen T, Janssen MAP, Halfens RJG. Malnutrition prevalence in the Netherlands: results of the annual Dutch National Prevalence Measurement of Care Problems. British Journal of Nutrition. 2009:101:417-432

15. Shahin ES, Meijers JMM, Schols JMGA, Tannen A, Halfens RJG, Dassen T.The relationship between malnutrition parameters and pressure ulcers in hospitals and nursing homes. Nutrition. 2010 Sep;26(9):886-9. doi: 10.1016/j.nut.2010.01.016. Epub 2010 May 4.

16. Van Nie-Visser NC, Schols JMGA, Lohrmann C, Meijers JMM, Meesterberends E, Halfens RJG. An international prevalence measurement of care problems: study protocol. Journal of Advanced Nursing 2013;69:E18-E29

17. Dijkstra A, Tiesinga LJ, Plantinga L, Veldman G, Dassen TWN. Diagnostic accuracy of the Care Dependency Scale. Journal of Advanced Nursing. May 2005 Volume 50, issue 4, pages 410-416. 
18. Halfens RJG, Meesterberends E, Nie-Visser NC van, Lohrmann C, Meyers JMM, Hahn S, Vangelooven C, Schols JMGA. International prevalence measurement of care problems: results. Journal of Advanced Nursing. 2013;69(9),e5-e17.doi:10.111/jan.12189

19. Gillespie BM, Chaboyer WP, McInnes E, Kent B, Whitty JA, Thalib L. Repositioning for pressure ulcer prevention in adults. Cochrane database of systematic reviews 2014, issue 4. No.:CD009958. DOI: 10.1002/14651858.CD009958.pub2

20. Moore Z, Cowman S. Pressure ulcer prevalence and prevention practices in care of the older person in the republic of Ireland. Journal of Clinical Nursing 2012 Feb;21(3-4):362-71. Doi:10.1111/j.13652702.2011.03749.x. Epub 2011 Jul 7.

21. Breimaier HE, Halfens RJG, Wilborn D, Meesterberends E, Haase Nielsen G, Lohrmann C. Implementation Interventions Used in Nursing Homes and Hospitals: A Descriptive, Comparative Study between Austria, Germany, and The Netherlands. ISRN Nursing Volume 2013 (2013), Article ID 706054, 12 pages. http:/ dx.doi.org/10.1155/2013/706054

22. Tooher R, Middleton P, Babidge W. Implementation of pressure ulcer guidelines: what constitutes a successful strategy? Journal of Wound Care. 2003 Nov;12(10):373-8, 380-2.

23. Meesterberends E, Halfens RJG, Lohrmann C, Schols JMGA. The implementation of pressure ulcer guidelines in Dutch and German nursing homes: a qualitative study. Theses Maastricht 2013

24. Meesterberends E, Halfens RJG, Lohrmann C, Schols JMGA, de Wit R. Evaluation of the dissemination and implementation of pressure ulcer guidelines in Dutch nursing homes. Journal of evaluation in clinical practice. March 2010, doi:10.1111/j.1365-2753.2010.01487.x

25. Demarré L, Vanderwee K, Defloor T, Verhaeghe S, Schoonhoven L, Beeckman D. Pressure ulcers: knowledge and attitude of nurses and nursing assistants in Belgian nursing homes J Clin Nurs. 2012 May;21(9-10):1425-34. doi: 10.1111/j.1365-2702.2011.03878.x. Epub 2011 Nov 1. 
O

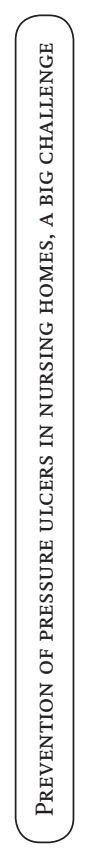




\section{CHAPTER 3}

PRESSURE RELIEF, COLD FOAM OR STATIC AIR?

A SINGLE CENTER, PROSPECTIVE, CONTROLLED

RANDOMIZED CLINICAL TRIAL IN A DUTCH

NURSING HOME

van Leen MWF, Hovius SER, Neyens JCL, Halfens RJG, Schols JMGA. 


\section{Abstract}

\section{Objective:}

At present, the evidence regarding the type of mattress that is the best for preventing pressure ulcers is not convincing. In a single center, prospective, controlled trial we compared a static air overlay mattress (no electric pump needed) on top of a cold foam mattress with a cold foam mattress alone on pressure ulcer incidence in nursing home residents.

\section{Methods:}

4083 residents were included in the study with a score lower than 12 points on the Norton scale and no pressure ulcer at the start of the study. 42 residents received a cold foam mattress and 41 residents received a static air overlay on top of that cold foam mattress. Out of bed we standardized the pressure reduction in sitting position by using a static air cushion in both groups. Residents were checked weekly in both groups for pressure ulcers.

Only when there were signs of developing a pressure ulcer grade 2 or higher, repositioning by our nursing home pressure ulcer protocol (PU protocol) was put into practice.

\section{Results:}

Seven residents (17.1\%) on a cold foam mattress and two (4.8\%) on a static air mattress developed a pressure ulcer grade 2 or more. There was no difference regarding pressure ulcer incidence between residents with a high risk (Norton 5-8) and residents with a medium risk (Norton 9-12). In 5 out of 7 residents who developed a pressure ulcer on a foam mattress the ulcers showed no healing using our PU protocol. In the static air group all pressure ulcers healed by regular treatment according to our PU protocol.

\section{Conclusions:}

In this study, static air overlay mattresses provided a better prevention than cold foam mattresses alone (4.8\% versus $17.1 \%$ ). The Norton scores of the residents in both groups did not change during the 6 month trial period. Our decision to use repositioning only when there were signs of a pressure ulcer seems to be acceptable when a static air overlay is in position. However, the score of $17.1 \%$ development (incidence) of pressure ulcers in the foam group may stress the need of repositioning when using only this type of mattress. 


\section{Introduction}

Pressure relieving systems are commonly used for prevention and treatment of pressure ulcers. To reduce the risk of pressure ulcers support surfaces can be used to redistribute pressure over a larger surface area of the patient's body can be used. The mostly used systems for prevention and treatment of pressure ulcers (PUs) are: cold foam mattresses, visco-elastic foam mattresses, alternating air mattresses, static air mattresses with or without an electric pump and air fluidized beds. Despite their widespread use in daily practice, there is in fact little scientific evidence supporting the use of these systems except expert opinion. It is suggested that a cold foam mattress is better than a standard hospital mattress. ${ }^{1,2,3}$ Therefore in many clinics and long-term care settings, already for years, standard $12-13 \mathrm{~cm}$ thick hospital mattresses (density foam $40 \mathrm{~kg} / \mathrm{m}^{2}$ ) have been replaced by cold foam mattresses. ${ }^{1,2,3}$

Cold foam mattresses are made of polyether foam. This is an elastic foam consisting of many very small closed air cells. After compression it recovers very quickly its original shape. The foam shows no "memory" behavior like the foam used for viscoelastic foam.

The quality of the mattress depends on the specific gravity of the foam. This can vary from 20 till 55 kilogram/cubic meter. Manufacturers can produce different types of stiffness.

Cold foam mattresses were also used in our nursing home De Naaldhorst in Naaldwijk, the Netherlands.

After 1994 no other studies are available about the use of cold foam mattresses to prevent pressure ulcers. ${ }^{4}$

Moreover, it is stated that using only a preventive mattress is ineffective for adequate PU prevention. Therefore in addition, universal guidelines $\left(\mathrm{NICE}^{5}, \mathrm{EPUAP}^{6}, \mathrm{NPUAP}^{7}\right.$, Dutch CBO guideline ${ }^{8}$ ) promote repositioning in bed every three hours in day time or for four hours at night.

In most guidelines there also is attention for relieving pressure and friction when patients are sitting in (wheel)chairs. Defloor studied which type of pressure reducing chair cushion showed the lowest maximum contact pressure. Static air cushions (Waffle®, Repose®) scored the best in a laboratory test. ${ }^{9}$ 
In nursing home residents prevention of PUs is very important; not only because of the frailty of this population but also because PUs considerably reduce quality of life.

Since 1998, Maastricht University conducts a National Prevalence Measurement of Care problems (called LPZ-measurement), including pressure ulcers, in hospitals, nursing homes, homes for the elderly and home care organizations. ${ }^{10}$ These annual measurements have clearly revealed that PUs in Dutch nursing homes are a relevant and important problem. Therefore, the prevalence of PUs has become an indicator of quality of care in Dutch nursing homes. ${ }^{11}$

Nursing home De Naaldhorst participates structurally in the yearly LPZ-measurement and showed in the period of 1999-2001 a mean overall prevalence of category 2, 3 and 4 PUs of $15 \%$.

In this nursing home 150 patients reside, who mostly are very old (mean age 83 years) and have complex somatic and/or psycho-geriatric problems.

In the period $1994-2001$ all residents were lying on a standard $15 \mathrm{~cm}$ cold foam mattress (Silhouette®, Comfortex, Winoma USA).

Furthermore, a pressure ulcer prevention protocol (PU protocol) was used, mainly based on the Dutch guidelines for prevention of pressure ulcers. ${ }^{8}$ Standard in this protocol is a daily check of the condition of the skin, if needed followed by appropriate measures to reduce pressure, e.g. the use of pressure relieving devices at the first signs of non-blanchable redness, extra attention for nutritional status and comorbidity.

However, contrary to the national guidelines, in our pressure ulcer prevention protocol (PU protocol) repositioning every 3 hours a daytime and every 4 hours at night is not structurally incorporated, because of 2 reasons. First: the interference in sleep during night time diminishes the quality of the residents' sleep and second: the associated higher workload of the nursing staff results in higher costs.

Only when other preventive measures are applied, like using another type of mattress/cushion, and when there are signs of non-blanchable erythema, reposition is standard in our PU protocol.

Siderenko et al. looked at the effects of using a static air overlay on sacral and heel pressure ulcers in a clinical population. ${ }^{12}$ One of the conclusions was a $5 \%$ development 
of PUs, when patients were laying on a static air mattress contrary to a standard hospital mattress ( $15 \%$ on a water mattress and $25 \%$ on an alternating mattress).

Based on the study of Sideranko et al and the study of Defloor (both mentioned above) we were very interested to perform a study looking at the efficacy of an additional static air overlay mattresses.

The main aim of this study was:

- To evaluate the clinical efficacy of combining a standard $15 \mathrm{~cm}$ cold foam mattress with a static air overlay mattress versus a cold foam mattress alone in preventing pressure ulcers.

\section{Methods}

A single center, prospective, controlled randomized clinical trial was performed in De Naaldhorst in the period March 2002 until October 2004.

All residents living in our nursing home with a Norton score below 13 at the start or during the study were asked to participate.

Inclusion criteria for residents were: age $>65$, a Norton score between 5-12 and informed consent of the residents or their representatives in case of mental disorders. The only exclusion criterion was a pressure ulcer in the previous 6 months.

To detect a clinically relevant reduction of the incidence of pressure ulcers from $10 \%$ to $2.5 \%$ with a statistical safeguard of $\alpha 0.05$ and a power of 0.80 , we calculated that 38 patients were needed in each group.

The Medical Ethical Committee of the Reinier de Graaf hospital in Delft approved the study.

To assess the risk of PUs we used the Norton scale. ${ }^{13}$ This scale measures PU risk; scores range from 1 to 4 regarding items like mental and physical condition, activity, mobility and incontinence. The maximum score is 20 (no risk at all) and the minimum score is 5 . A score lower than 14 indicates patients at risk for pressure ulcers. (a score of 9-12 indicates a medium risk and 5-8 a high risk).

Randomization into two groups was performed after informed consent using numbered envelopes: 
A. a control group receiving a standard cold foam mattress

B. an intervention group receiving a combination of that mattress with a static air overlay.

When out of bed, all residents were sitting on a static air pillow following the institutional PUPP. At night, nobody received repositioning conform this PU protocol.

Subsequently, both groups of included residents were followed for a period of 6 months.

A weekly inspection of the skin to assess the possible occurrence of a skin lesion was done by an independent nurse. The Norton scale was registered for every resident at 44 the start and the end of the observation period.

During the intervention period, the primary outcome parameter was the development of category 2, 3 and 4 pressure ulcers (EPUAP-classification ${ }^{9}$ ) at the heel or in the sacral/hip region. No repositioning was allowed before development of a category 2 pressure ulcer.

\section{Statistical analyses}

All statistical analyses were performed using SPSS 15.0 for Windows (SPSS Inc., Chicago, IL USA).

\section{Results}

Eighty-three residents were included, 41 received a cold foam mattress (control group) and 42 a static air overlay mattress upon the cold foam mattress (intervention group). The baseline characteristics of the residents in both groups are shown in table 1.

In total 9 residents died ( 5 in the cold foam group and 4 in the static air group) during the study period. In all cases, there was no relation with the study activities. None of the residents who died developed a pressure ulcer.

Table 1 shows that at the start of the study more residents in the static air group had a very low Norton score, indicating that in the intervention group there were more pressure ulcer prone residents. No further relevant differences were encountered between both groups. 
Table 1. Demographics of all residents $(n=83)$

\begin{tabular}{|c|c|c|c|c|}
\hline \multirow{2}{*}{$\begin{array}{l}\text { Residents } \\
\text { Characteristics at start study }\end{array}$} & \multicolumn{2}{|c|}{$\begin{array}{l}\text { Cold foam (control group) } \\
\text { (41) }\end{array}$} & \multicolumn{2}{|c|}{$\begin{array}{l}\text { Static Air + Cold foam } \\
\text { (intervention group(42) }\end{array}$} \\
\hline & $\mathbf{N}$ & $\mathbf{N} \%$ & $\mathbf{N}$ & $\mathbf{N} \%$ \\
\hline Age in years (mean $\pm S D$ ) & $83.1 \pm 7.86$ & & $81.1 \pm 8.37$ & \\
\hline Gender (females) & 34 & $82.9 \%$ & 33 & $78.6 \%$ \\
\hline Norton $5-8$ at start of study & 22 & $53.7 \%$ & 26 & $61.9 \%$ \\
\hline Norton 9-12 at start of study & 19 & $46.3 \%$ & 16 & $38.1 \%$ \\
\hline \multicolumn{5}{|l|}{ Diagnoses } \\
\hline Dementia & 31 & $75.6 \%$ & 31 & $73.8 \%$ \\
\hline CVA & 4 & $9.8 \%$ & 8 & $19.0 \%$ \\
\hline Rheumatoid arthritis & 0 & $0.0 \%$ & 1 & $2.4 \%$ \\
\hline Encephalopathy & 1 & $2.4 \%$ & 0 & $0.0 \%$ \\
\hline m. Parkinson & 1 & $2.4 \%$ & 1 & $2.4 \%$ \\
\hline Diabetes & 1 & $2.4 \%$ & 0 & $0.0 \%$ \\
\hline Arthrosis & 1 & $2.4 \%$ & 0 & $0.0 \%$ \\
\hline Hip fracture & 1 & $2.4 \%$ & 1 & $2.4 \%$ \\
\hline COPD & 1 & $2.4 \%$ & 0 & $0.0 \%$ \\
\hline Died during study 1 & 5 & $12.2 \%$ & 4 & $9.5 \%$ \\
\hline Norton $5-8$ at end of study & 21 & $58.3 \%$ & 26 & $68.4 \%$ \\
\hline Norton 9-12 at end of study & 15 & $41.7 \%$ & 12 & $31.6 \%$ \\
\hline
\end{tabular}

${ }^{1}$ none of the deceased residents developed a pressure ulcer during participation

Table 2 shows that the pressure ulcer incidence in the intervention group was lower than in the control group ( 2 versus $7 ; \mathrm{p}=0.088$ Fisher's Exact Test). The confidence interval related to the difference between the two groups varied from $1.3 \%$ to $25.9 \%$.

Table 2. number of residents who developed a grade 2, 3 or 4 pressure ulcer in the six month observation period

\begin{tabular}{|l|c|c|c|c|}
\hline & \multicolumn{2}{|c|}{$\begin{array}{c}\text { Cold foam } \\
\text { (control group) }\end{array}$} & \multicolumn{2}{|c|}{$\begin{array}{c}\text { Static air + cold foam } \\
\text { (intervention group) }\end{array}$} \\
\hline Norton & $5-8$ & $9-12$ & $5-8$ & $9-12$ \\
\hline Grade 2 & 0 & 2 & 0 & 1 \\
\hline Grade 3 & 4 & 1 & 1 & 0 \\
\hline Grade 4 & 0 & 0 & 0 & 0 \\
\hline
\end{tabular}

Five residents on the cold foam mattress showed progression of the ulcers to category 3 or 4 pressure ulcer and had to be transferred onto another type of bed (low air loss), while two residents with a category 3 were treated by standard care by 
PU protocol on the cold foam mattress with a good healing after standard care with additional repositioning every 3-4 hours.

The two residents, lying on the static air mattress were both treated successfully as well with standard care by protocol on the static air mattress including additional repositioning every 3-4 hours.

\section{Discussion}

The main aim of this study was to evaluate the clinical efficacy of a combination of a standard $15 \mathrm{~cm}$ cold foam mattress with a static air overlay mattress versus that cold foam mattress alone on the incidence of pressure ulcers in nursing home residents.

In this study $4.8 \%$ PUs occurred in the static air group and $17.1 \%$ in the cold foam group, which means that the cold foam mattress only appears to be associated with a higher risk for development of a pressure ulcer than combining a foam mattress with a static air overlay.

The results suggest a rather clear relationship between the type of mattress and the pressure ulcer risk, which was significant at $\mathrm{p}<0.10$.

After our study we performed an additional search in Medline and Cinahl over the period January 2001-September 2009 to look for studies comparing cold foam mattresses and static air overlay mattresses (Mesh terms: prevention, pressure ulcer, cold foam mattresses, static air mattresses). No studies were found.

Therefore we advise to perform a larger study to assess the reproducibility and significance of our findings.

Taking every individual patient into account, it is very difficult to make a choice for a special type of pressure relieving mattress. In the last Dutch guideline of 2002 no special advice for different types of patients is recommended as well. One of the most important reasons for this is the fact that the effects of pressure reduction are based on studies with mainly young and healthy people. ${ }^{8}$

In the recently published guidelines of the NPUAP/EPUAP (September 2009) again no special advice is stated that helps us to make a tailor-made choice. ${ }^{14}$ Hopefully future studies will provide more specific guidance on this. 
An interesting finding of our study was that the results seem to support our policy to use a static air overlay mattress without repositioning at day or night time.

Of course it remains an ethical question whether we may take up in our PU protocol: no repositioning.

Especially because most international PU guidelines as well as our own national guideline incorporate repositioning as an essential preventive measure. But there is more. Measurements for prevention are based on two items: relieving pressure by limiting the time of pressure, but maybe even or more important relieving pressure by enlargement of the area. If we look to the evidence for the effect of repositioning, most evidence is based on studies by Defloor and Vanderwee., ${ }^{9,15 .}$

They showed that even when repositioning is used, $9-16 \%$ of the patient developed a pressure ulcer grade 2 or more.

In this study we found an incidence of $4.8 \%$ without repositioning. Maybe our choice for just using enlargement is enough on this type of static air mattresses. Nevertheless further studies are required to conform our preventive strategy in patients lying on static air mattresses and to provide more evidence for the value of using repositioning during the use of different types of mattresses.

Moreover, the results of the control group in our study in fact stressed the necessity of embedding continous repositioning again in our PU protocol when using a cold foam mattress alone.

Based on this study, however, we decided to change our PU protocol in another way. When non-blanchable redness of the skin is noticed by the nursing staff, additional pressure relief by using a static air overlay has become a standard procedure now.

This study was performed without conflicts of interest.

We thank the director as well as the medical and nursing staff of the Naaldhorst in Naaldwijk for making this study possible. 


\section{Conflicts of interest}

The study and writing of the article is done without any financial support by a company. The companies are not informed before, during or after the study and writing of the article. 


\section{REFERENCES}

1. Hofman A, Geelkerken RH et al. Pressure ulcers and pressure-decreasing mattresses: controlled clinical trial. Lancet 1994;343(8897):568-571.

2. Goldstone LA, Norris M, O'Reilly M, White J. A clinical trial of a bead bed system for the prevention of pressure ulcers in elderly orthopedic patients. Journal of Advanced Nursing. 1982;7:545-548.

3. Gray DG, Campbell M. A randomized clinical trial of two types of foam mattresses. Journal of Tissue Viability. 1994;4:128-132.

4. Cullum N, McInnes E, Beller-Syer SEM, Legood R. Support surfaces for pressure ulcer prevention (Cochrane Review). The Cochrane Library 2006;(1): Oxford: update software.

5. NICE guideline for risk assessment and prevention of pressure ulcer 2003.

6. EPUAP prevention and treatment guidelines, 1998.

7. NPUAP. Pressure ulcer prevalence, costs and risk assessment. Decubitus 1989; 2(2):24-8

8. Dutch CBO guideline pressure ulcers 1992, Utrecht, The Netherlands.

9. Defloor T. Pressure reduction and repositioning for prevention of pressure ulcers. Thesis University of Gent 2000.

10. Halfens RJG, Meyers JMM, Neyens JCL, Schols JMGA. Landelijke Prevalentiemeting Zorgproblemen 2009 (National Prevalence Measurement of care problems 2009, Universitaire Pers Maastricht (www.lpz-um. eu/index.html?SF=metingen $2009 \& S I=\& S T=1 \& i d=10$ )

11. Van Veen JAH. Toetsingskader Verantwoorde Zorg (Audit cadre reponsable care). Health Care Inspectorate, 2006 (www.igz.nl/?view=Search+results)

12. Sideranko S, Quinn A, Burns K, Froman RD. Effects of position and mattress overlay on sacral and heel pressure ulcers in a clinical population. Research in Nursing and Health 1992;15(4):245-251.

13. Norton D. Calculating the risk: reflections on the Norton Scale. Advances in Wound Care 1989;9:38-43.

14. NPUAP/EPUAP prevention and treatment guidelines 2009. www.epuap.org

15. Vanderwee K. The effect of pressure relieving measurements. Thesis University of Gent, 2006. 
O

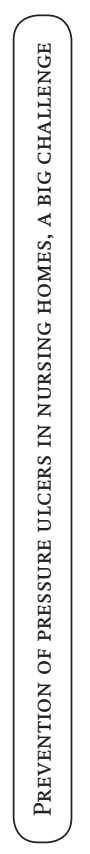




\section{CHAPTER 4}

PRESSURE RELIEF WITH VISCO-ELASTIC FOAM OR WITH COMBINED STATIC AIR OVERLAY?

A PROSPECTIVE, CROSS-OVER, RANDOMIZED

CLINICAL TRIAL IN A DUTCH NURSING HOME

van Leen MWF, Hovius SER, Halfens RJG, Neyens JCL, Schols JMGA. 


\section{Abstract}

\section{Objective:}

Evidence of the best mattress for preventing pressure ulcers is not conclusive. In a single center, prospective, cross-over trial on pressure ulcer incidence in nursing home residents, a static air overlay mattress, without a pump, on top of a viscoelastic foam mattress was compared with a visco-elastic foam mattress alone.

\section{Methods:}

The study was performed using a randomized crossover design. Forty-one residents with a score of 19 or lower on the Braden scale, but with no pressure ulcer at the start, were divided in 2 groups; 21 residents received a visco-elastic foam mattress (control group) and 20 residents a static air overlay on top of a visco-elastic foam mattress (intervention group) for the first 6 months. In the second (crossover) period of 6 month, 19 residents participated in both groups. Residents were checked weekly and, only when signs of development of a pressure ulcer where present was treatment altered to reposition residents according to the nursing home pressure ulcer protocol. No statistically significant differences were noted between the 2 groups with regard to age, gender, or Braden score.

\section{Results:}

Of 41 residents, 3 died and were unable to participate in the crossover period, 8 residents (22.2\%) developed a category 2 or higher pressure ulcer on a visco-elastic foam mattress (control group) and $2(5.2 \%)$ on a static air mattress (intervention group) ( $\mathrm{p}=0.087$ ). They developed a pressure ulcer category 2 or more. There was a difference regarding pressure ulcer incidence between residents with a very low Braden score between 6 and 12, and residents with a mean score between 13-19. Out of 8 residents, in the 2 (25\%) who developed a pressure ulcer on a foam mattress, the ulcers showed no signs of healing. In the static air group all pressure ulcers healed by normal treatment according to our pressure ulcer treatment protocol.

\section{Conclusions:}

In this small study, static air overlay mattresses provided a better prevention than visco-elastic foam mattresses alone (5.2\% vs $22.2 \%$ ). The Braden scores of the patients in both groups did not change during the 6-month test. The decision to use 
repositioning only when there were signs of a pressure ulcer is acceptable when a static air overlay is in position. The $22.2 \%$ incidence of pressure ulcers in the foam group, however, may stress the need of continuing repositioning when using this type of mattress.

Key words: prevention, pressure ulcer, visco-elastic foam, static air overlay 


\section{Introduction}

Pressure-relieving mattresses are commonly used for prevention and treatment of pressure ulcers (PUs). To reduce the risk of PUs, clinicians and nurses may use support surfaces to redistribute pressure over a larger surface area of the patient's body. Despite their widespread use in daily practice, there is in fact little scientific evidence supporting the use of these systems, except expert opinion. By 1984, it was already suggested that a cold foam mattress provides better PU prevention than a standard hospital mattress ${ }^{1,2,3}$. Therefore, in many clinics and long-term care settings, for many years, standard 120-130 mm thick hospital mattresses (density foam $40 \mathrm{~kg} / \mathrm{m}^{2}$ ) were replaced by cold foam mattresses. ${ }^{1,2,3}$ In 2002 the Dutch Institute for Healthcare Improvement (CBO) guideline is revised. ${ }^{4}$ Based on studies of Defloor, the guideline advised to change the standard care to a visco-elastic foam mattress instead of a cold foam mattress. ${ }^{5}$

Cold foam mattresses are made of polyether foam, an elastic foam composed of many very small closed air cells, that recovers original shape quickly after compression. Visco-elastic foam mattresses are made of polyurethane foam, an elastic foam that consists of many open-air memory cells. When a patient is in the supine position the foam transforms its shape in a few seconds, which is called envelopment.

Until 2002 the authors of this study used a cold foam mattress in De Naaldhorst, a nursing home in Naaldwijk, the Netherlands. The mattress was used mostly in combination with a static air overlay (Repose®, Frontier Therapeutics Blackwood, South Wales) when there was a non-blanchable redness present on a patient for more than 6 hours. This time period allowed for a more accurate diagnosis of category I PU.

After the Dutch guideline was published in 2002, the new recommendations concerning the type of pressure-relieving mattress were implemented. Additionally, guidelines from the National Institute for Health and Care Excellence (NICE), the European Pressure Ulcer Advisory Council (EPUAP), the National Pressure Ulcer Advisory Panel (NPUAP), and the CBO guideline, state that using only a preventive mattress is not enough for adequate PU prevention. Universal guidelines also promote repositioning in bed every 3 (day time) or every 4 hours (night time) as a standard procedure for patients at risk. ${ }^{6,7,8}$ In the PU protocol of the Naaldhorst however, repositioning is only started after development of a PU category I or higher. This is because the workload of repositioning is very high and there is less evidence for a standard inclusion of repositioning. In a nursing home PU prevention is important, not only because of the frailty of the population, but also because PUs 
considerably reduce residents' quality of life. Unfortunately, more than $20 \%$ of the residents in Dutch nursing homes still develop a PU during their stay. ${ }^{9}$ Therefore, in the Netherlands, the prevalence of PUs has become an official indicator of quality of care in nursing homes.

Every April since 1998, Maastricht University (Maastricht, The Netherlands) conducts the National Prevalence Measurement of Care Problems (LPZ), including PUs in hospitals, nursing homes and home care organizations. ${ }^{9}$ Nursing home de Naaldhorst participates structurally in the LPZ-measurement.

At the time of the present study, 150 patients resided in the Naaldhorst, with a mean age of 83 years, and were admitted due to complex somatic and/or psycho geriatric problems. After changing the PU protocol and introducing the standard visco-elastic mattresses (Duosmart®, Kabelwerk Eupen, Eupen, Belgium) between 2002 and 2004, the prevalence of PUs category 2 and higher rose from $4.1 \%$ to $12.4 \%$. During the same period the nursing home was involved in a randomized clinical trial of the effectiveness of a cold foam mattress versus the same mattress combined with a static air overlay. Data analyses showed a better result for the patients laying at the overlay ( $4.8 \%$ versus $17.1 \%$ category 2 or more). ${ }^{10}$

Furthermore a pressure ulcer prevention protocol (PUPP) is used, mainly based on the Dutch guideline for prevention of pressure ulcers. ${ }^{4}$

Contrary to the national guideline in our PUPP repositioning every 3-4 ours during the night is not structurally incorporated, because of 2 reasons. First: the interference in sleep during night time diminishes the quality of the residents' sleep and second: the associated higher workload of the nursing staff results in higher costs. In a RCT done in our clinic between 2002 and 2005 we measured 4.8\% development of pressure ulcers, when residents were lying on a static air overlay without using repositioning by protocol. Data of the LPZ also showed that repositioning is used as a standard of care in only $18-20 \%$ of nursing homes in the Netherlands, due to the increased work load of the nursing staff and the wish to avoid the disturbance of patients during their sleep. In the afore mentioned study, repositioning was also only performed after development of a PU and it appeared that, even then, a low percentage of PUs occurred when patients were lying on a static overlay. ${ }^{10}$

Taking into account the issues of an observed increase of PU prevalence after the start of using visco-elastic mattresses alone and our strategy regarding the application of repositioning, the authors wanted to study the efficacy of additional static air overlay 
mattresses upon a visco-elastic mattress. It is not possible to change from a viscoelastic mattress to a static air mattress alone because static air mattresses are only available as an overlay type. Just before the start of this study, an additional search in Medline and CINAHL over the period January 2001-September 2006 was performed, using the following Mesh terms: "prevention of pressure ulcers", "pressure ulcer", "visco-elastic foam mattresses" and "static air mattresses". No studies comparing visco-elastic foam mattresses and static air overlay mattresses were found. Moreover, it was expected that, by using a static air overlay, the frequency of repositioning might be lowered in this case as well.

Therefore, the aim of this study was to evaluate the clinical efficacy of a combination of a standard $15 \mathrm{~cm}$ visco-elastic foam mattress with a static air overlay mattress versus a standard $15 \mathrm{~cm}$ visco-elastic foam mattress alone in preventing PUs.

\section{Methods}

A single center, prospective, cross-over, randomized clinical trial was performed. The crossover design was based on the aim to perform the total study in one nursing home so that all data could be collected by one researcher. Moreover, the authors wanted all residents to receive the same treatments for the same periods. This type of design has two advantages: 1) the influence of confounding covariates is reduced because each crossover resident serves as his or her own control. 2) crossover designs are statistically efficient and so require fewer subjects than non-crossover designs. Selected residents were asked to participate one year (6 months on each type of mattress). The first cohort started for 6 months on a visco-elastic foam mattress only (control group) and for the next 6 months on a static air overlay mattress placed on top of a visco-elastic foam mattress (intervention group). The second cohort started for 6 months on a static air overlay mattress placed upon a visco-elastic foam mattress and for the next 6 months on a viscoelastic foam mattress only.

Inclusion criteria for residents were: age $>65$, a Braden score between 6 and 19, and informed consent of the residents or their representatives in case of mental disorders (dementia). Patients were only excluded if they had an existing pressure ulcer. Forty residents were required to detect a clinically relevant reduction of the incidence of PUs from $12 \%$ to $4 \%$ with an $\alpha$ of 0.05 and a power of 0.80 . The Braden scale was used to assess the risk of PUs. ${ }^{11}$ This scale measures PU risk, by assigning scores from 1 to 4 regarding mental and physical condition, activity, mobility and incontinence. (Each category is rated on a scale of 1 to 4 , excluding the 'friction and shear' category, which is rated on a 1-3 scale, for a possible total of 23 points.) The 
maximum score of 23 indicates no risk of PU development, and a minimum score of 7 indicates a high risk of PU development. A score of 13-19 indicates a medium at risk for PUs, and a score of 7-12 a high risk. ${ }^{11}$

Residents were randomized into two groups after informed consent using numbered envelopes. Group A was the control group, receiving a standard visco-elastic foam mattress, and group B was the intervention group, receiving a combination of a standard visco-elastic foam mattress with a static air overlay. When out of bed, all residents sat on a static air pillow accordingly PUPP. In compliance with standard PU protocol at the Naaldhorst, study participants did not receive repositioning unless a category I PU developed. Residents skin was inspected weekly to assess the possible occurrence of a skin lesion. Residents were evaluated using the Braden scale at the beginning of the study and after 6 months. During the intervention period, the primary outcome parameter was the development of category 2, 3 or 4 PUs (EPUAP-classification ${ }^{7}$ ). Category I was excluded because of the inconclusiveness of the diagnosis. In this cross-over design, transfer to another type of mattress was carried out after a period of six months. All included residents, except 3 who died, participated in the second part of the study. Subsequently, both groups of included residents were followed for in total a period of 12 months. The Medical Ethical Committee of the Reinier de Graaff hospital in Delft approved the study. At that time there was no obligation to take up the study in the trial register.

\section{Statistical analyses}

All statistical analyses were performed using SPSS 17.0 for Windows (SPSS Inc., Chicago, IL USA). Differences were tested with a 2-sided Fisher exact test for categorical variables and Students t-test for continuous variables. Residents, who died during the first period, were included in the analysis of the first period but not of the second period. Residents who died during the second period were included in the analysis of the second period.

\section{Results}

Forty-one residents were included at the start of the study, of which 38 were able to participate in the second crossover part of the study. The baseline characteristics of the residents are shown in table 1 . In total, 5 residents died during the study period ( 3 in the first 6 month and 2 in the second). In all cases, there was no relation with the study activities. Only one of the residents developed a PU, but died acutely caused by a heart attack. The causes of death of the other 4 residents during the 
trial were dehydration $(n=3)$, (caused by patients' refusal to drink fluids while still in nursing home), and pneumonia ( $\mathrm{n}=1)$.

Table 1. Demographics of all patients (41)

\begin{tabular}{|l|c|c|c|}
\hline Characteristics at baseline & $\begin{array}{c}\text { Control Group } \\
\mathbf{N}=\mathbf{2 1}\end{array}$ & $\begin{array}{c}\text { Intervention Group } \\
\mathbf{N}=\mathbf{2 0}\end{array}$ & \begin{tabular}{c} 
P-value \\
\hline Age in years (mean \pm SD)
\end{tabular} \\
\hline Gender (females) & 18 & 79,1 & $\mathrm{P}=.243$ \\
\hline Disease (dementia) & 18 & 14 & $\mathrm{P}=.224$ \\
\hline CVA & 3 & 16 & $\mathrm{P}=.697$ \\
\hline Braden first period score 6-12 score 13-19 & 14 & 4 & $\mathrm{P}=.530$ \\
\hline \multicolumn{1}{|c|}{ score13-19 } & 7 & 11 & $\mathrm{P}=1.00$ \\
\hline Died in first period* & 2 & 9 & $\mathrm{P}=.486$ \\
\hline Died in second period & 0 & 1 & 2 \\
\hline Braden second period score 6-12 & 13 & 10 & $\mathrm{P}=.508$ \\
\hline
\end{tabular}

*Only 1 of the deceased residents developed a pressure ulcer during participation.

Table 1 shows that no significant differences were found between both groups, although in group 1 more residents were female, and more residents had a very low Braden score, indicating that in the intervention group there were more pressure ulcer prone patients.

Table 2 shows that the PU incidence in the intervention group was lower than in the control group ( 2 vs 8 [5.2\% versus $22.2 \%]$; $=.087$ ). The confidence interval related to the difference between the two groups was $0.92 \%$ till $23.4 \%$

Eight residents in the control group developed a PU, 5 were in the heel region. One ulcer occurred in the intervention group in the pelvic region and 1 on the heel. The majority of the PUs $(n=8)$ developed when residents had a Braden score lower than 14; the other two residents had scores of 14 and 15. All PUs that developed during the first 6-months study period were healed before the mattress was changed for the second 6-month period. Two residents using a visco-elastic foam mattress developed category 3 PUs and had to be transferred onto a low air loss bed, according to protocol, due to deterioration of their wounds. There was no reason to change the mattresses for all other residents. Wound healing was successful after starting standard wound care protocol. Because of non-blanchable redness, repositioning was started in the control group for 9 patients (22.5\%) and in the intervention group 
for 1 patient (2.5\%) ( $\mathrm{p}=.014)$. Confidence interval related to the difference between the two groups was $1.325 \%$ till $91.887 \%$.

Table 2. Incidence of pressure ulcers (category II and higher) per condition

\begin{tabular}{|c|c|c|c|c|c|c|c|c|}
\hline & & \multicolumn{3}{|c|}{$\begin{array}{l}\text { Visco-elastic foam mattress } \\
(\mathrm{n}=40)^{*}\end{array}$} & \multicolumn{3}{|c|}{$\begin{array}{l}\text { Visco-elastic foam mattress } \\
\text { with a static air overlay } \\
\text { mattress }(n=39)^{* *}\end{array}$} & \multirow{3}{*}{\begin{tabular}{|l} 
P-value \\
$P=.087$
\end{tabular}} \\
\hline \multicolumn{2}{|c|}{ Total amount of pressure ulcers } & \multirow[t]{2}{*}{8} & \multicolumn{2}{|c|}{ Location } & \multirow[t]{2}{*}{2} & \multicolumn{2}{|c|}{ Location } & \\
\hline & & & pelvic region & heel & & pelvic region & heel & \\
\hline \multirow[t]{3}{*}{ Category } & 2 & 6 & 2 & 4 & 2 & 1 & 1 & \\
\hline & 3 & 2 & 1 & 1 & 0 & 0 & 0 & \\
\hline & 4 & 0 & 0 & 0 & 0 & 0 & 0 & \\
\hline \multicolumn{2}{|c|}{ Repositioning (yes) } & \multicolumn{3}{|l|}{8} & \multicolumn{3}{|l|}{1} & $P=.014$ \\
\hline
\end{tabular}

\section{Discussion}

The aim of this study was to evaluate the clinical efficacy of a combination of a standard $15 \mathrm{~cm}$ visco-elastic foam mattress alone vs a standard $15 \mathrm{~cm}$ visco-elastic foam mattress with a static air overlay mattress on the incidence of PUs in nursing home residents. In this study, a visco-elastic foam mattress resulted in a higher risk for developing a PU (22.2\%) than when this mattress was combined with a static air overlay (5.2\%). In spite of the power analyses, there was not a statistical significance $(\mathrm{p}=0.087)$.

When signs of non-blanchable redness are evident, the results demonstrate a reduction in the development of pressure ulcers category 2 or higher when using a static air mattress on top of a visco-elastic foam mattress as opposed to the use of a visco-elastic foam mattress alone. An explanation for this difference may be the way of reduction of the perpendicular and tangential component of force at the contact area. On the static air overlay both strengths (i.e., the reduction in the development of category 2 PUs and the reduction of the perpendicular and tangential component of force) are less than on foam. Air mattresses are also able to realize more immersion and envelopment which may result in a better contact of the mattress over a larger skin area. ${ }^{12}$

In 2012, a study of Vermette et al. looked at the effects of using an alternating system (one for patients $<200$ pounds and one for patients $>200$ pounds) versus a static air has been published about the effects of the use of an alternating system ( 2 types; 
one for patients below 200 pounds and one for over 200 pounds) versus a static air overlay. ${ }^{13}$ Patients were followed for a period of 2 weeks, had a Braden score $<14$ and received repositioning every 2 hours. In the alternating group 6 patients (12\%) developed a PU and in the static air overlay group 2 patients (4\%). Despite a total of 105 participating patients no statistical significance was reached $(\mathrm{P}=0.1269)$, but the trend is comparable, and in agreement with the current study.

The question that remains is when does repositioning by protocol needs to be included? Current international and national guidelines advise repositioning every 3 or 4 hours in the international and national guidelines. ${ }^{4,5,6,7}$ This recommendation is based on 2 studies with patients lying on a visco-elastic foam mattress. ${ }^{5,14}$ There are no RCTs on other types of mattresses available. A systematic review of pressure ulcer prevention strategies found insufficient evidence to support a specific regimen like a change of position every 2 or 4 hours. ${ }^{15}$ It was concluded that an effective repositioning regimen will be indicated by the absence of persistent erythema over bony prominences. If persistent erythema occurs, this may require more frequent repositioning or as decided in the authors' strategy, only repositioning when there are signs of non-blanchable redness. The results of this study support the policy to use a static air overlay mattress without repositioning. However, the results of the control group confirmed the necessity of repositioning when using a viscoelastic foam mattress, which stresses the importance to follow the international guidelines. ${ }^{4,6,7,8}$

\section{Limitations}

The limitations of this study are mainly related to the crossover design. First, there is the issue of "order" effects, because it could be possible that the order in which the mattresses were used, may have affected the outcome. Second, there could have been a "carry-over" effect between both interventions, which may have confounded the estimates of the intervention effects. Third, because the study in fact was underpowered, replication with a larger number of patients will be necessary to confirm our conclusion that a static air overlay on top of a viscoelastic foam mattress results in a much better prevention of PU than a visco-elastic foam mattress.

\section{Conclusion}

In this small study, a visco-elastic foam mattress with a static air overlay provided better prevention than a visco-elastic foam mattress alone (5.2\% vs $22.2 \%$ ). The Braden scores of the patients in both groups did not change during the 6-months test. The decision to use repositioning only when there were signs of a pressure ulcer 
is acceptable when a static air overlay is in position. The $22.2 \%$ incidence of pressure ulcers in the foam group, however, may stress the need to continue repositioning when using this type of mattress.

After this study, and also based on the results of a cold foam vs static air mattress study, ${ }^{10}$ the institution where this study was conducted chose to make use of a static air overlay as part of its standard PU prevention protocol. For every at-risk patient lying on a cold foam or visco-elastic foam mattress, the static air overlay was added as a second step instead of repositioning at De Naaldhorst nursing home.

\section{Acknowledgment}

The authors wish to thank the director and medical and nursing staff of De Naaldhorst, Naaldwijk, The Netherlands, without whose approval and support this study would not have been possible. 


\section{REFERENCES}

1. Hofman A, Geelkerken RH, Wille J, Hermans J, Breslau PJ,et al. Pressure sores and pressure-decreasing mattresses: controlled clinical trial.Lancet 1994;343:568-571

2. Goldstone LA, Norris M, O'Reilly M, White J. A clinical trial of a bead bed system for the prevention of pressure sores in elderly orthopaedic patients. J Adv Nurs 1982;7:545-548

3. Gray DG, Campbell M. A randomized clinical trial of two types of foam mattresses. Journal of Tissue Viability 1994;4:128-132

4. CBO. Prevention and treatment of pressure ulcers, a Dutch guideline, Utrecht 2002 http://www.diliguide. nl/richtlijnen/professionals/huidaandoeningen/. Accessed October 1,2013

5. Defloor T. Pressure reduction and repositioning for prevention of pressure sores [thesis]. Ghent. Belgium: Ghent University; 2000

6. National Institute for Clinical Excellence. Clinical guideline: 7 Pressure Ulcer Prevention. Pressure ulcer risk assessment and prevention, including the use of pressure-relieving devices (beds, mattresses and overlays) for the prevention of pressure ulcers in primary and secondary care. http://www.nice.org.uk/ nicemedia/live/10928/29179/29179.pdf. Published October 2003.Accessed September 19, 2013

7. European Pressure Ulcer Advisory Panel and National Pressure Ulcer Advisory Panel. Treatment of pressure ulcers: quick reference guide. http://www.epuap.org/guidelines/Final_Quick_Treatment.pdf. Accessed September 19,2013.

8. Verpleegkundigen \& Verzorgenden Nederland. Guideline Prevention and treatment of pressure ulcers. Published November 2011. http://wwdiliguide.nl/richtlijnen/professionals/huidaandoeningen/. Assessed October 2013

9. LPZ. National prevalence measurement care problems www.lpz-um.eu/media/text/Home/LPZ rapport_2012.pdf Published 2012.Accessed September 19,2013

10. van Leen M, Hovius S, Neyens J, Halfens R, Schols J. Pressure relief, cold foam or static air? A single center, prospective, controlled randomized clinical trial in a Dutch nursing home. J Tissue Viability $2011 ; 20,30-34$

11. Bergstrom N, Braden BJ, Laguzza A, Holman V. The Braden scale for predicting pressure sore risk. Nurs. Res.1987;36(4):205-210.

12. Pressure Ulcer Expert Working Group. International review. Pressure ulcer prevention: pressure, shear, friction and microclimate in context. A consensus document. Wounds Int. 2010;2-10.http://www. woundsinternational.com/pdf/content_8925.pdf. Published September 19,2013

13. Vermette S, Reeves I, Lemaire J. Cost effectiveness of an air-inflated static overlay for pressure ulcer prevention: a randomized, controlled trial. Wounds.2012;24:207-214.

14. Vanderwee K. The effect of pressure relieving measurements [thesis].Ghent,Belgium: Ghent University; 2006.

15. Reddy M, Gill SS, Rochon PA. Preventing pressure ulcers: a systematic review. JAMA 2006;296(8):974-984 
O

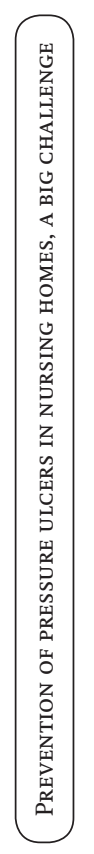




\section{CHAPTER 5}

\section{The Effect of A Simple 3-STEP Pressure}

Relieving Strategy for Preventing

PRESSURE UlCERS:

AN ExPloRATIVe Longitudinal STUdy From

2002-2011

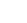

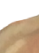




\section{Abstract}

\section{Objective:}

Pressure ulcers (PUs) still form an important and distressing problem in Dutch nursing homes. Pressure ulcer prevention protocols are generally based on current guidelines. The authors developed an alternative 3-step protocol to help prevent pressure ulcers. The effects of this new 3-step protocol on the prevalence of pressure ulcers in residents at risk of developing PUs in the Avoord nursing homes in EttenLeur/Zundert (Netherlands) were calculated. In addition, the protocol's general cost effects were explored.

\section{Method:}

Data on the prevalence of PUs and the use of preventive measures were derived from the annual independent International Prevalence Measurement of Care Problems of Maastricht University (Landelijke Prevalentiemeting Zorgproblemen [LPZ]). This annual measurement was implemented in 1998 and measures care problems such as the prevalence of PUs and related preventive measures. Data on residents at risk of developing PUs at the Avoord nursing homes in Etten- Leur/Zundert (Netherlands) were analysed and compared with national data between 2002 and 2011.

\section{Results:}

The introduction of the 3-step protocol resulted in a significant reduction of the nosocomial prevalence of category $2-4$ PUs. The prevalence was reduced from $8.7 \%$ to $0.5 \%$ during the first year and remained stable at about $2 \%$ throughout the rest of the study period. The prevalence at the national level also decreased during this period, but not as much, and was still significantly higher in 2011. The use of alternated systems decreased to almost $1 \%$. Use of static air mattresses showed an almost linear rise in the Avoord nursing homes from the start of the implementation of the protocol, while the trend for both types of mattresses remained stable on a national level. Introducing the static air mattress instead of the more expensive alternating mattresses helped to reduce the mean daily costs of mattresses at the Avoord nursing homes by more than $70 \%$ compared to national figures. The workload of the nursing staff decreased as well due to the reduction of repositioning. 


\section{Conclusion:}

The introduction of the 3-step protocol showed to be effective. The prevalence of PUs and the mean daily costs were reduced to, and have been sustained at, a significantly lower level.

Key words: pressure ulcer, prevention, static air overlay, visco-elastic foam mattresses 


\section{Introduction}

A pressure ulcer (PU) is a localized injury to the skin and/or underlying tissue, usually over a bony prominence that results from pressure, including pressure associated with shear. ${ }^{1,2}$ Pressure ulcers are an important and distressing care problem in nursing homes, leading to suffering and loss of residents' quality of life. Pressure ulcers are also associated with considerable extra health care costs

The prevalence of category 1-4 PUs in Dutch nursing homes is more than $20 \%$, which indicates the relevance of the problem. ${ }^{3}$ Severens et al. calculated that the costs of PU care in nursing homes related to extra hours of nursing and medical care, pressure relieving mattresses, and prolonged stays in the nursing home, amounts to more than 500 million euros a year. ${ }^{4}$ Another study calculated the mean costs for the treatment of category 2-4 PUs to be $€ 750$ per ulcer in 2001, based on materials and activities of nurses and doctors. ${ }^{5}$ The mean costs are currently $€ 1,670$ per ulcer, based on the price level of 2012. No cost analyses have been conducted in nursing homes in the Netherlands in the past 10 years.

In addition to the main causative factors, a number of contributing or confounding factors have been associated with the occurrence of PUs. These include mobility problems, neurological diseases, perfusion/oxygenation, skin moisture, body temperature, general health, and nutritional status. ${ }^{4}$

Pressure-relieving systems play an important role in the daily practice of PU prevention. Physicians and nurses often apply additional support surfaces to redistribute pressure over a larger surface area of the patient's body. Although there is little scientific evidence to support the use of these systems, they are widely used. ${ }^{6}$

A higher specification foam mattress should be used for patients with a high risk of developing a PU rather than a standard hospital foam mattress. ${ }^{6}$ Since 2002, guidelines on PUs, such as the Dutch Institute for Healthcare Improvement (CBO) guidelines, have recommended as a primary step that a visco-elastic foam mattress be used in combination with repositioning in bed every 3 hours during the day or every 4 hours during the night. ${ }^{7,8,9,10}$ If this measure does not meet the needs of the patient, use of an alternating mattress is advised. ${ }^{78,9}$ When using another type of mattress, additional repositioning must be taken in consideration. ${ }^{78,9}$

Every year since 1998, Maastricht University has conducted a national prevalence measurement (Landelijke Prevalentiemeting Zorgproblemen [LPZ]) in hospitals, nursing homes, and home care organizations. The LPZ is a cross-sectional, multi- 
center prevalence measurement that uses a standardized questionnaire to record data such as the prevalence and risk of pressure ulcers and the preventive and therapeutic measurements taken in daily PU care. ${ }^{11}$ Participation in the LPZ enables nursing homes to compare their PU prevalence, PU prevention, and PU policy with national data from the entire nursing home sector.

The Avoord Zorg en Wonen nursing homes (Etten- Leur/Zundert, the Netherlands) participate in these annual LPZ measurements and PU prevalence data are available from 2002 until the present. In 2002, 2003, and 2004, the prevalence of category 1-4 PUs in the Avoord Zorg en Wonen nursing homes was 30.7\%, 26.6\%, and $22.2 \%$, respectively. These data are comparable with the mean national prevalence data from 2002, 2003, and 2004. The decrease from $30.7 \%$ to $22.2 \%$ was probably the result of all basic mattresses being replaced by visco-elastic foam mattresses in 2002 , the first step recommended by the Dutch national guidelines. In 2002, the Avoord nursing homes also started using a standard protocol for PU prevention, based on the CBO 2002 guidelines, that includes repositioning patients every 3-4 hours.

National and international guidelines often include repositioning as a structural part of preventive measures. However, in daily practice in the Netherlands compliance is low, with very few patients actually repositioned every 3-4 hours; in fact, only $15 \%-30 \%$ of the patients at risk were repositioned in the years 2002- 2004 (Figure 1). Around 2002, following the findings of Defloor, many guidelines on PUs, including the $\mathrm{CBO}$ guidelines, based their advice for repositioning on a visco-elastic foam mattress. ${ }^{10}$ The current international guidelines from the National Pressure Ulcer Advisory Panel (NPUAP), the European Pressure Ulcer Advisory Panel (EPUAP) and Verpleegkundigen \& Verzorgenden Nederland (V\&VN) also based the use of repositioning on these studies, because other studies before December 2007 were analysed as methodologically insufficient. ${ }^{11}$ The guidelines also specify that repositioning should be considered when patients have other types of mattresses. However, 2 studies by van Leen et al. that involved residents at high risk for PUs, as measured with the Norton and Braden scales, showed the incidence of pressure ulcer development was approximately $4 \%$ for residents using a static air mattress without repositioning, while the findings of Defloor showed a $15 \%$ incidence. ${ }^{10,12,13}$

The original PU prevention protocol of Avoord Zorg en Wonen was revised to implement a more effective protocol. The revisions were based on studies by Defloor, Van Leen et al., and Sideranko et al., who studied the effects of static air. ${ }^{10.12 .13 .14}$ 
Figure 1. Effect of 3-step PU prevention protocol on PU prevalence in Avoord nursing homes versus the national nursing home sector and the effects on the percentage of repositioning (2002-2011).

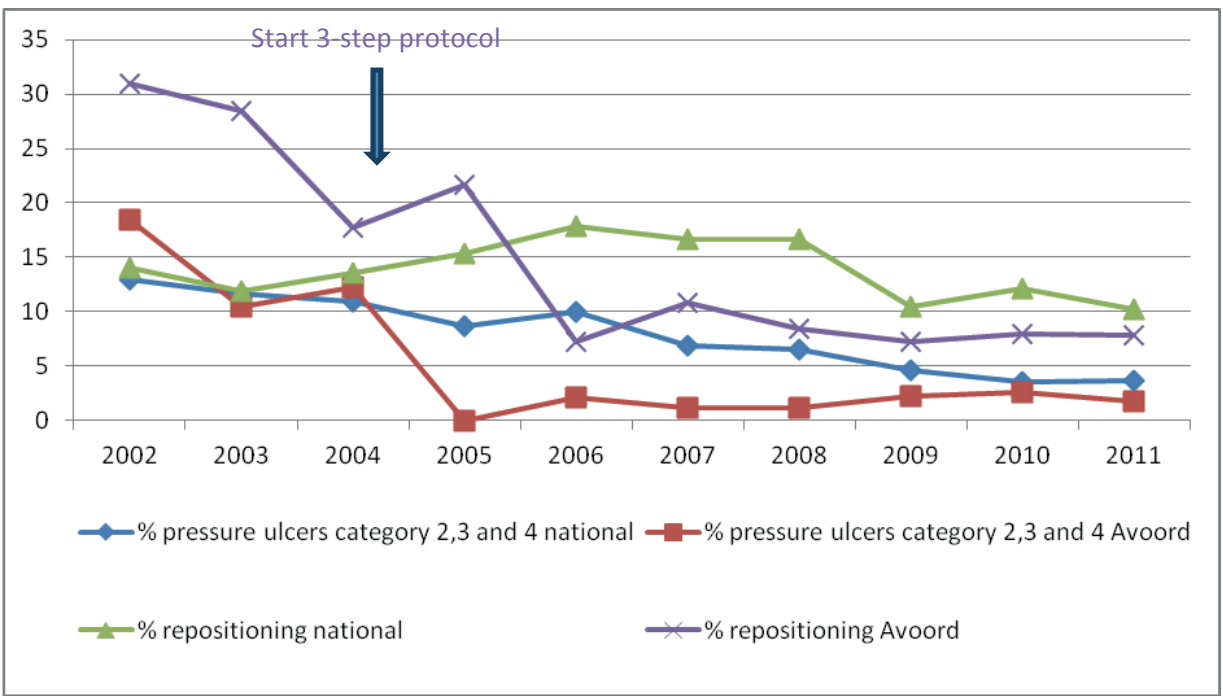

Pressure ulcers: 2005-2009 $p<0.001,2010 p<0.002$ and $2011 p<0.001$

Repositioning: $2005 p=005,2006 p<0.001,2007 p=0.007,2008 p=0.001,2009 p=0.013,2010 p=0.003$ and 2011 $\mathrm{p}=0.049$

The new PU protocol contains 3 main steps:

1) all residents receive a standard visco-elastic mattress, with no repositioning

2) residents who develop signs of a possible category $1 \mathrm{PU}$ (non-blanchable redness) receive a static air overlay (Repose, Frontier Therapeutics Ltd, Blackwood, South Wales UK) in addition to the visco-elastic foam mattress, again with no repositioning;

3) residents who still develop a PU are repositioned every 3 hours during the day and every 4 hours during the night. If this 3-step protocol is insufficient because a resident develops a more severe PU (i.e. category 4) the patient's mattress will be swapped for a low-air loss system.

The yearly participation in the LPZ measurements between 2002 and 2011 allowed for the evaluation of resident outcome effects and the cost effectiveness of using this new protocol, and an ability to compare the PU prevalence figures with the national prevalence figures in the nursing home sector. 
The authors aimed to address the following research questions in this study:

1) What effect did the implementation of the 3-step PU prevention protocol have on the nosocomial PU prevalence in the Avoord nursing homes from 2005 to 2011 ?

2) How does the nosocomial PU prevalence in the Avoord nursing homes from 2005 to 2011 compare to national figures from the nursing home sector during those same years?

3) What indication can be given about the cost-effectiveness of the implementation of static air mattresses and the reduction of alternating systems between 2005 and 2011 per 100 patients, compared to national data?

\section{Method}

\section{Design of the study}

This study aimed to investigate the effect of a 3-step prevention protocol. Longitudinal data was collected starting in April 2002. The study followed a longitudinal design with annual measurements (2002-2011) of the nosocomial PU prevalence and the use of preventive measures. An annual comparison with figures from the national nursing home sector was also made.

\section{Participants}

The participants were all residents at risk of developing PUs (Braden $\leq 20$ ) in the Avoord Zorg en Wonen nursing homes. In the Netherlands, all residents living in nursing homes participate in the LPZ without need for specific informed consent. The medical ethical committee of the Maastricht University decided, in cooperation with the government, that specific consent is not necessary for collecting this data. In practice, the authors inform their residents that they collect the data by a yearly letter. All residents at risk of developing PUs in Dutch nursing homes who participated in the annual LPZ measurements were also included in the study, with the exception of residents who received palliative care, short term rehabilitation, or who stayed in the nursing homes for less than 30 days.

\section{Instruments}

Data about gender, age, prevalence of PUs, type of mattress, and repositioning were derived from the LPZ measurements from 2002 to 2011. The LPZ uses a standardized questionnaire to register data on matters such as the risk of and the prevalence of PUs, and the preventive and therapeutic measurements undertaken in daily PU care, including the type of preventive mattresses used. The risk of PUs is measured 
with the full Braden scale score. ${ }^{15}$ Braden scores range from 1 to 4 based on 6 items concerning sensory perception, moisture, activity, nutritional intake, and friction and shear. The maximum score is 23 (no risk at all). A score lower than 20 indicates the patient is at risk of PUs. ${ }^{16}$ The original PU grading system of the EPUAP was used to classify the PUs., ${ }^{4,9}$

Overall cost was calculated using the mean price of a leasing contract for the 7 most commonly used alternating mattress systems; the actual cost for the static air overlay mattresses was used. Prices for leasing alternating systems ranged between $€ 7$ and $€ 16$ per day. Based on information gathered by the authors from 4 large companies that lease $80 \%$ of the alternating mattress systems in the Netherlands, a mean price of $€ 10$ per day was used for the cost estimation. Static air mattresses are not available for leasing in the Netherlands, and the cost per mattress was $€ 175$. The authors' experience with the static air overlay mattresses shows that 1.4 mattresses are generally needed per patient each year. The mean cost for 100 patients was calculated.

\section{Interventions}

In 2002 residents at the Avoord nursing homes all received a visco-elastic foam mattress as a standard prevention measure. In January 2005, the new 3-step model (i.e., basic visco-elastic foam mattress, static air overlay, repositioning) was introduced. The nursing staff was trained and subsequently coached by a specialist wound nurse for 3 months.

\section{Statistical Analysis}

All statistical analyses were performed using the statistical package for social science version 20.0 (IBM SPSS Statistics for Windows, Armonk, NY). Logistic regression analyses were used to test the differences in the prevalence of pressure ulcers developed in the Avoord clinics and in the other participating nursing homes.

\section{Results}

As shown in Table 1, there is a large fluctuation in the number of residents who participated in the LPZ at a national level. This is largely because of the voluntary nature of participation in the LPZ and because not all nursing homes participated during the whole study period. The increase of Avoord residents is based on its organizational increase of available beds. As shown in Table 1, the mean age of the participating residents was approximately 80 years, and there was no statistical difference between the participating residents at a national level and those residing at the Avoord nursing homes. 
The results of the national nursing home sector show a linear reduction of the nosocomial prevalence of category 2-4 PUs from 8.7\% in 2005 to $3.7 \%$ in 2011 (Table 1). After the introduction of the new 3-step model at the Avoord nursing homes in 2005, the PU nosocomial prevalence dropped to $0.5 \%$ within 1 year. This level was maintained at a rate of between $1.2 \%$ and $2.6 \%$ (category $2-4$ ) throughout the rest of the study period. In the period since the implementation of the new 3-step protocol in 2005, the PU prevalence rate has been significantly lower in the Avoord nursing homes than in the overall national nursing home sector.

Although the national PU guidelines recommend that residents should be repositioned, this is not a standard procedure in Dutch nursing homes, as shown in Figure 1. The national annual data show a small fluctuation in repositioning percentages between $10 \%$ and $17 \%$. The data from Avoord nursing homes show a decline from $22 \%$ in 2005 to $7 \%$ in 2006 , and a trend of stabilization in the years following that.

Table 1. Characteristics of participating residents at risk of PUs in the Avoord nursing homes and in all participating Dutch nursing Homes

\begin{tabular}{|c|c|c|c|c|c|c|c|}
\hline \multicolumn{2}{|c|}{ Residents at risk } & \multirow{2}{*}{$\begin{array}{r}\text { Number } \\
4600\end{array}$} & \multirow{2}{*}{\begin{tabular}{r|}
$\begin{array}{r}\text { Gender } \\
\text { (\% female) }\end{array}$ \\
73.1
\end{tabular}} & \multirow{2}{*}{$\begin{array}{r}\text { Mean age } \\
80.9\end{array}$} & \multirow{2}{*}{$\begin{array}{c}\text { Nosocomial prevalence } \\
\text { pressure ulcers Cat 2-4 } \\
13.0\end{array}$} & \multirow{3}{*}{$\begin{array}{r}\text { p-value } \\
0.923\end{array}$} & \multirow{3}{*}{$\begin{array}{r}95 \% \mathrm{Cl} \\
0.694-1.498\end{array}$} \\
\hline \multirow{2}{*}{2002} & All & & & & & & \\
\hline & Avoord & 114 & 77.2 & 83.0 & 18.5 & & \\
\hline \multirow{2}{*}{2003} & All & 4389 & 72.2 & 80.7 & 11.6 & \multirow{2}{*}{ * } & \multirow{2}{*}{ * } \\
\hline & Avoord & 169 & 73.1 & 81.4 & 10.4 & & \\
\hline \multirow{2}{*}{2004} & All & 7321 & 71.7 & 81.4 & 10.9 & \multirow{2}{*}{0.155} & \multirow{2}{*}{$0.548-1.100$} \\
\hline & Avoord & 172 & 70.9 & 82.6 & 12.2 & & \\
\hline \multirow{2}{*}{2005} & All & 8337 & 71.6 & 81.3 & 8.7 & \multirow{2}{*}{$<0.001$} & \multirow{2}{*}{$0.032-0.228$} \\
\hline & Avoord & 183 & 74.3 & 82.6 & 0.5 & & \\
\hline \multirow{2}{*}{2006} & All & 9829 & 71.4 & 82.1 & 10.0 & \multirow{2}{*}{$<0.001$} & \multirow{2}{*}{$0.120-0.403$} \\
\hline & Avoord & 190 & 76.3 & 82.8 & 2.1 & & \\
\hline \multirow{2}{*}{2007} & All & 14852 & 70.5 & 81.3 & 6.9 & \multirow{2}{*}{$<0.001$} & \multirow{2}{*}{$0.092-0.431$} \\
\hline & Avoord & 189 & 80.4 & 84.5 & 1.1 & & \\
\hline \multirow{2}{*}{2008} & All & 9533 & 70.8 & 80.8 & 6.5 & \multirow{2}{*}{$<0.001$} & \multirow{2}{*}{$0.089-0.453$} \\
\hline & Avoord & 179 & 81.6 & 82.2 & 1.2 & & \\
\hline \multirow{2}{*}{2009} & All & 12673 & 74.9 & 82.6 & 4.6 & \multirow{2}{*}{$<0.001$} & \multirow{2}{*}{$0.144-0.544$} \\
\hline & Avoord & 285 & 81.0 & 82.2 & 2.2 & & \\
\hline \multirow{2}{*}{2010} & All & 12503 & 75.7 & 83.1 & 3.5 & \multirow{2}{*}{0.002} & \multirow{2}{*}{$0.178-0.675$} \\
\hline & Avoord & 303 & 78.7 & 83.3 & 2.6 & & \\
\hline \multirow{2}{*}{2011} & All & 5750 & 73.6 & 83.1 & 3.7 & \multirow{2}{*}{0.001} & \multirow{2}{*}{$0.171-0.654$} \\
\hline & Avoord & 286 & 76.6 & 83.1 & 1.8 & & \\
\hline
\end{tabular}

* $=$ not all data available for a statistical analysis. 
Figure 2 shows data for the most commonly used types of mattresses for residents who did not receive enough pressure relief from a visco-elastic mattress and/or repositioning, a standard measure in the current national guidelines. ${ }^{7}$ In Dutch nursing homes during the study period, an average of $11.7 \%$ (ranging from $9.7 \%$ 14.3\%) of the residents at risk received an alternating mattress and 9.2\% (ranging from $6.8 \%-12.4 \%$ ) a static air mattress, indicating that about $20 \%$ of the residents received these 2 types of additional mattresses (alternating or static air) for extra PU prevention. Comparable data for the Avoord nursing homes show an average of $2.3 \%$ (ranging from $0.4 \%-5.4 \%$ ) received alternating mattresses and 13.1\% (ranging from $0 \%-25.4 \%$ ) received static air mattresses in addition to a visco-elastic foam mattress. This means that $15.4 \%$ of the residents received these 2 types of mattresses. Differences in the use of alternating systems and static air overlay mattresses between national and Avoord nursing homes were all significant $(P<0.001)$.

Figure 2. Use of alternating systems and static air systems in Avoord nursing homes versus the national Dutch nursing home sector (2002-2011).

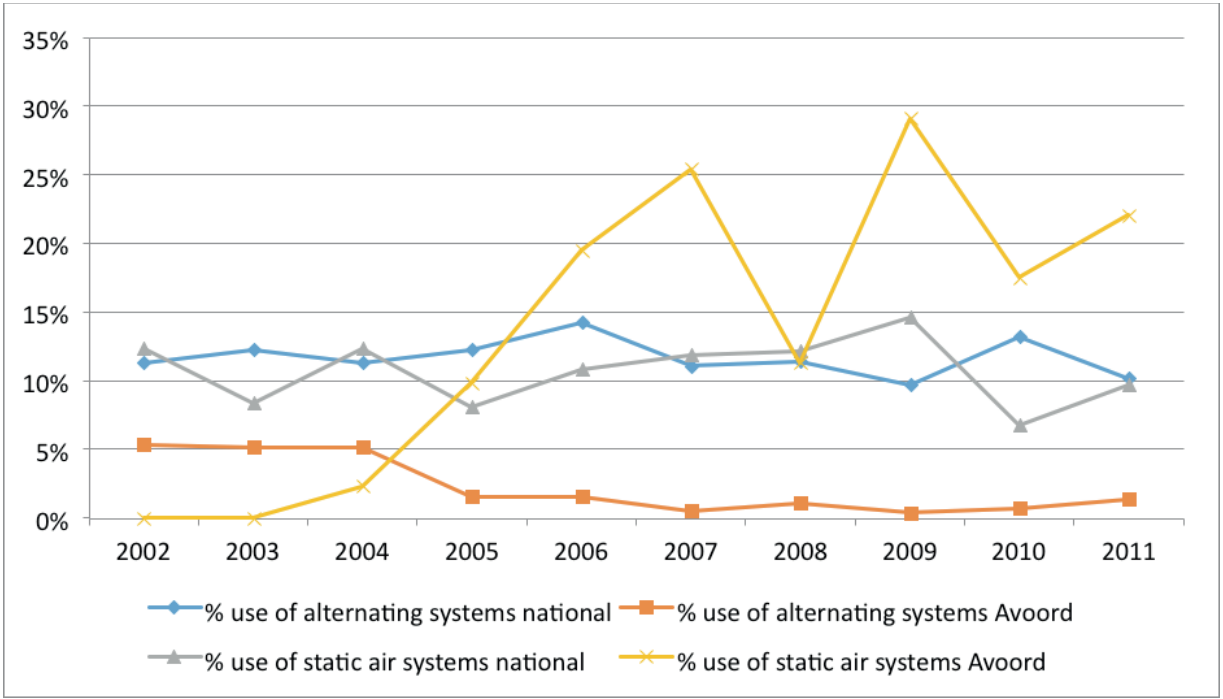

Alternating systems:2005,2006, 2010 and $2011 p<0.001,2007 p=0.002,2008 p=0.001,2009 p=0.001$ Static air overlay: $2005-2011 p<0.001$

\section{Discussion}

The main aim of this study was to evaluate the clinical efficacy of the introduction of the 3-step PU prevention protocol in Avoord nursing homes. The results showed a sustained decrease of PU prevalence after the implementation of the new protocol, which indicates the effectiveness of the 3-step protocol. However, a prospective 
and randomized incidence study is needed to confirm these results. The data from the national nursing home sector also showed a decline in PU prevalence, but was less pronounced than in the Avoord nursing homes. This can be explained by the growing attention for PU prevention in the Netherlands over the past decade.

The 3-step protocol also resulted in a decrease of repositioning from $22 \%$ to $7.8 \%$ in the Avoord nursing homes.

Residents are generally repositioned to reduce the duration and magnitude of pressure on vulnerable areas of the body and also to contribute to comfort, dignity, and functional ability. ${ }^{4}$ However, repositioning is very expensive for nursing homes because almost $50 \%$ of the patients in the authors' clinics who are repositioned have to be turned by 2 people. The task of repositioning patients also causes a considerable physical burden on the nurses and often interrupts patients' privacy and causes them discomfort (interruption of daily rhythm and sleep).

Current guidelines still incorporate repositioning as an essential and ethical preventive measure. In addition, a relevant question remains whether there is enough evidence-based data to make repositioning a standard measure. Defloor, Vanderwee and Moore et al showed that, even when $100 \%$ of patients are repositioned and receive a visco-elastic foam mattresses, 9\%-16.4\% still develop a category 2 or higher PU. ${ }^{10,17,18}$

The prevalence data from Avoord nursing homes show that after the 3-step PU prevention protocol was introduced, repositioning declined to $7.8 \%$ from 2006 to 2011 , and only $2 \%$ of the participatingresidents had a category 2 through $4 \mathrm{PU}$ on the days of measurement. Based on 2 earlier studies in the same population that led to the introduction of the 3-step protocol, the authors decided that in this protocol, standard repositioning combined with a high quality mattress was not basic prevention. ${ }^{12,13}$

Figure 1 clearly shows that repositioning is not always used for patients at risk in the Netherlands and that, as a result of the 3-step protocol, repositioning was used significantly less in the study nursing homes than in other nursing homes from 2006 on. This may imply that not all residents need repositioning for prevention of PUs. This study also reveals that using the 3-step protocol (with repositioning only taking place in step 3) may lead to a considerable improvement of PU prevalence. 


\section{Limitations}

This study had a number of limitations. Because the methodology involved a prevalence study, no causal conclusions could be made. Unfortunately, no incidence data are available in Dutch nursing homes concerning nosocomial PUs, so to monitor the effect of the 3-step protocol the authors chose to use data from the annual LPZ, which follows an internationally accepted methodology. ${ }^{19}$ Furthermore, only the Avoord nursing homes used the integrated prevention strategy (3-step protocol). No information was available on whether specific prevention strategies were used by the other Dutch nursing homes that participated in the LPZ; it was only known that these nursing homes followed the national guidelines.

Nevertheless, this study indicates that, thanks to the prolonged measurements over the period 2006-2011, the 3-step strategy may be more effective than the prevention strategy based on the 2002 national guidelines. Also, no revisions have been made to prevention strategies in the most recent national guidelines on PU prevention, based on the guidelines of the NPUAP/EPUAP since 2002. ${ }^{4,20}$ There are no changes in the strategy of prevention in later guidelines and all the preventive strategies in this study are based on the 2002 guideline.

The 3-step model uses the total Braden scale score. For the best chance of detecting all patients at risk, all patients with an overall score of less than 20 were included. ${ }^{15}$ A limitation of using a score of less than 20 is the fact that patients who are more active or mobile may be included as being at risk, which may introduce some bias to the data. Coleman et al. suggest that using specific items from the scale concerning activity and mobility might provide a better risk score. ${ }^{21}$ Although in theory the main cause of a PU is pressure, and PUs cannot develop without pressure, the development of PUs in real-life conditions is much more complicated. Other factors, such as malnourishment and sensory perception, also play a role. For instance, a malnourished patient with no mobility or activity limitations can develop a PU when the patient's sensory perception is limited, resulting in not enough position changes overnight. The Dutch guidelines therefore recommend the use of the fullscale score. 
Figure 3. Daily costs of alternating systems + static air overlay systems in Avoord nursing homes versus the national Dutch nursing home sector (2002-2011).

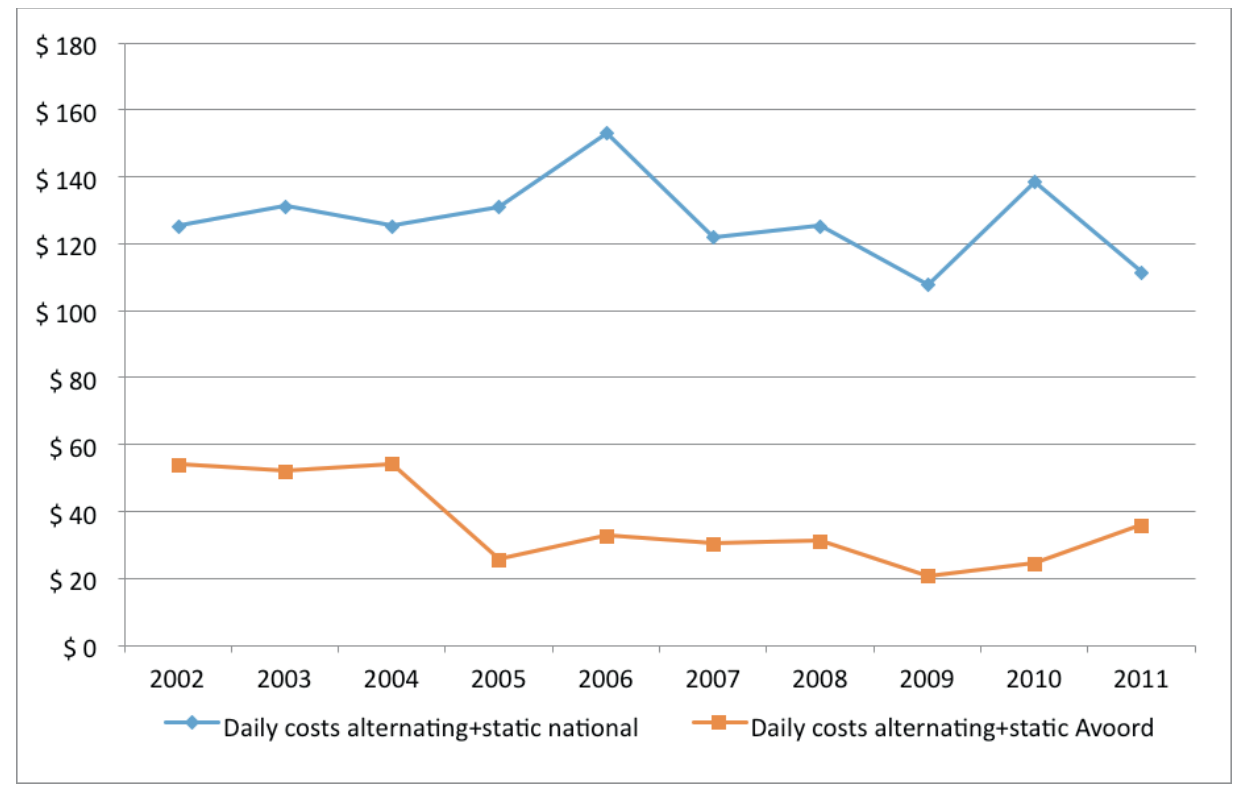

A final limitation in this study is that the calculation of costs is based on the costs of measurements taken during the day. A prospective cohort study is needed to give a more reliable statement of costs.

\section{Conclusion}

The overall conclusions of this study show the implementation of the new 3-step prevention protocol in the Avoord nursing homes resulted in: a sustained reduction of the prevalence of PUs; a considerable cost reduction in the use of preventive mattresses when not following the national PU guidelines (which may be followed at the discretion of individual facilities); and a considerable reduction of the workload for the nursing staff because fewer residents need repositioning with a secondary cost reduction resulting from a safe decrease of the application of repositioning. 


\section{REFERENCES}

1. European Pressure Ulcer Advisory Panel and National Pressure Ulcer Advisory Panel.Prevention and treatment of Pressure Ulcers 2009. www.epuap.com

2. Expert Working Group. International review. Pressure ulcer prevention: pressure, shear, friction and microclimate in context. A consensus document. London: Wounds International, 2010. http://www. woundsinternational.com/pdf/content_8925.pdf

3. Halfens RJG, Schols JMGA, Bartholomeyczik S, et al. International Prevalence Measurement of Care Problems (LPZ): 2009-2010 At a glance. Maastricht, Netherlands: Datawyse; 2011. http://www.lpz-um.eu/ media/text/home/Brochure_LPZ_2009-2010_At\%20 a\%20glance_21x21_v09\%20(2).pdf

4. Severens JL, Habraken JM, , Duivenvoorden S, Frederiks C. The cost of illness of pressure ulcers in the Netherlands. Adv Skin Wound Care. 2002;15(2):72-77.

5. Müller E, van Leen MW, Bergemann R. Economic evaluation of collagenase-containing ointment and hydrocolloid dressing in the treatment of pressure ulcers. Pharmacoeconomics. 2001;19(12):1209-1216.

6. McInnes E, Jammali-Blasi A, Bell-Syer S, Dumvill J, Cullum N. Support surfaces for pressure ulcer prevention. Cochrane Database of Syst Rev. 2011; (4). doi:10.1002/14651858.CD001735.pub4.

7. Dutch Insitute for Healthcare Improvement (CBO). Prevention and treatment of pressure ulcers. 2002. Available by request from www.cbo.nl.

8. European Pressure Ulcer Advisory Panel. Prevention and Treatment of Pressure Ulcers. EPUAP Rev. 1999;1:31-33. Available by request from www.epuap. org.

9. National Institute for Health and Care Excellence. Pressure ulcer prevention: pressure ulcer risk assessment and prevention, including the use of pressure-relieving devices (beds, mattresses and overlays) for the prevention of pressure ulcers in primary and secondary care. NICE guidelines CG7. http://www.nice.org.uk/guidance/cg7. Published October 2003.

10. Defloor T. Pressure Reduction and Repositioning for Prevention of Pressure Sores [thesis]. Ghent, Belgium: University of Ghent; 2000.

11. Halfens RJG, Schols JMGA, Meijers JMM, et al. National Prevalence Measurement of Care Problems [in Dutch]. Maastricht, Netherlands: Caphri School for Public Health and Primary Care; 2010. http://www. stuurgroepondervoeding.nl/fileadmin/dbc/documenten/LPZ_rapport_2010_v69.pdf.

12. van Leen M, Hovius S, Neyens J, Halfens R, Schols J. Pressure relief, cold foam or static air? A single centre, prospective, controlled randomized clinical trial in a Dutch nursing home. J Tissue Viability. 2011;20(1);30- 34 .

13. van Leen M, Hovius S, Neyens J, Halfens R, Schols J. Pressure relief, visco-elastic foam or static air? A single centre, prospective, cross-over randomized clinical trial in a Dutch nursing home. WOUNDS. 2013;25(10)287-292.

14. Sideranko S, Quinn A, Burns K, Froman RD. Effects of position and mattress overlay on sacral and heel pressure ulcers in a clinical population. Res Nurs Health. 1992;15(4):245-251.

15. Halfens RJ, Van Achterberg T, Bal RM. Validity and reliability of the Braden scale and the influence of other risk factors: a multi-center prospective study. Int J of Nurs Stud. 2000;37(4):313-319.

16. Bergstrom N, Braden BJ, Laguzza A, Holman V. The Braden scale for predicting pressure sore risk. Nurs Res. 1987;36(4):205-210. 
17. Vanderwee K. The Effect of Pressure Reducing Methods in Relation to Pressure Ulcers [thesis]. Ghent, Belgium: University of Ghent; 2006.

18. Moore Z, Cowman S, Conroy RM. A randomised controlled trial of repositioning, using the $30^{\circ}$ tilt, for the prevention of pressure ulcers. Journal of Clinical Nursing. 2011;20(17-18):2633-2644.

19. van Nie-Visser NC, Schols JM, Meesterberends E, Lohrmann C, Meijers JM, Halfens RJ. An international prevalence measurement of care problems: study protocol. Journal of Advanced Nursing. 2013;69(9):e1829.

20. Verpleegkundigen \& Verzorgenden Nederland. Dutch national guidelines for prevention and treatment of pressure ulcers [in Dutch]. http://www.venvn.nl/LinkClick.aspx?fileticket=8TrtENI2ZjE\%3D\&tab id=1852. Published November 2011.

21. Coleman S, Gorecki C, Nelson A, et al. Patient risk factors for pressure ulcer development: systemic review. Int J Nurs Stud. 2013;50(7):974-1003. 
O

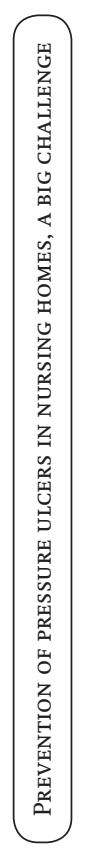


ChAPTER 6

PreVentive EFFECT OF A SKIN INTERFACE MICROCLIMATE REGULATING SYSTEM ON PRESSURE ULCER DEVELOPMENT:

A MULTICENTER, PROSPECTIVE, CONTROLLED RANDOMIZED CLINICAL TRIAL IN DUTCH NURSING HOMES

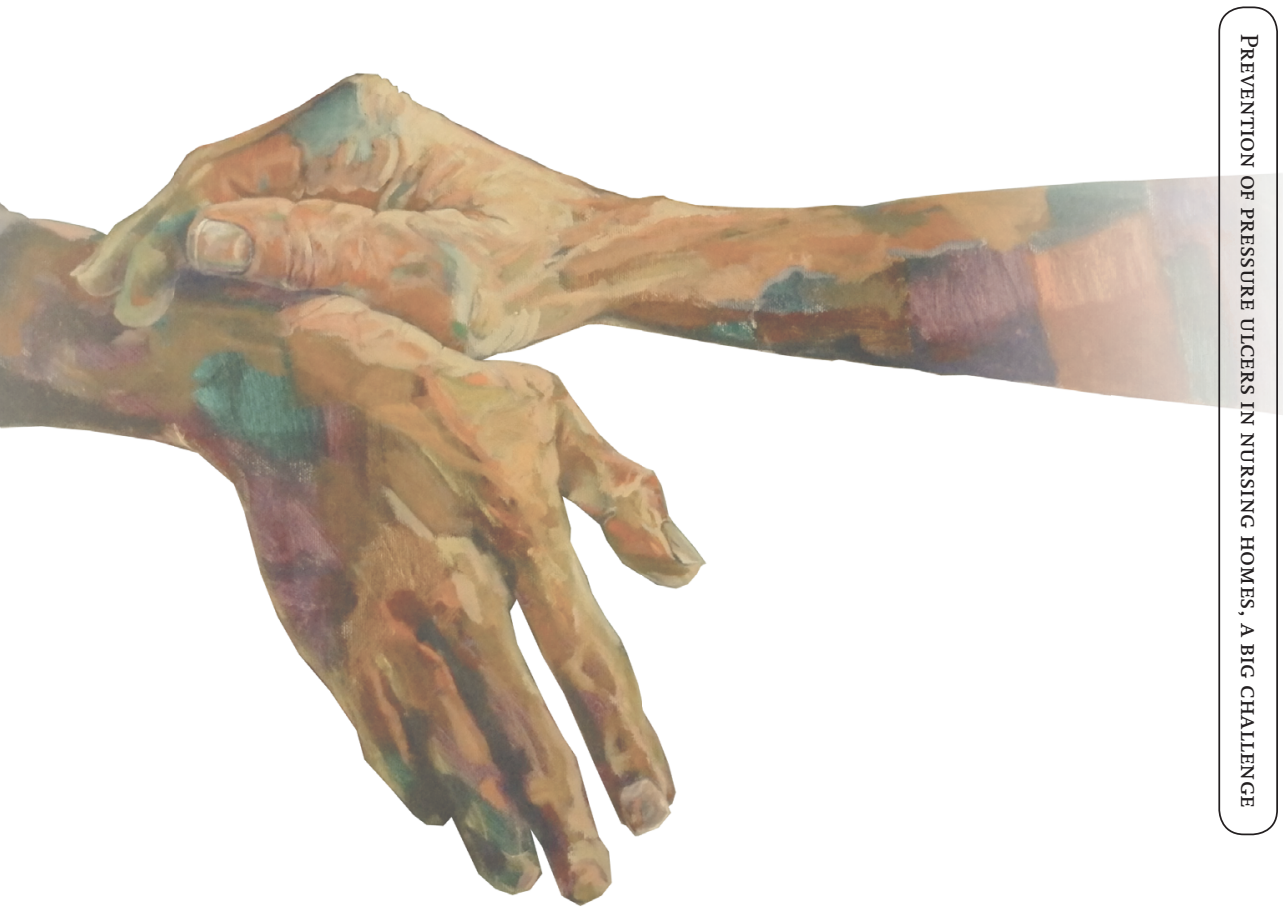

van Leen MWF, Halfens RJG, Hovius SER, Schols JMGA. 


\section{Abstract}

\section{Objective:}

Prevention of pressure ulcers (PUs) is based on pressure relief, diminishing shear stress and control of the microclimate. Based on the recommendations in the most recent EPUAP/NPUAP/PPPIA guideline for prevention of PUs 2014 , a pressure relieving, shear stress diminishing and skin interface microclimate controlling multilayer support system has been developed for use on top of a visco-elastic foam mattress. Aim of this study was to test the PU preventive effect of this system compared to the use of a visco-elastic foam mattress alone.

\section{Methods:}

A multicentre, prospective, controlled randomized clinical trial was executed in 21 nursing homes. Residents with a Braden score less than 16; a life expectancy of more than 3 months and no PU during the last 3 months were asked to participate and included after informed consent. Residents were divided into two groups. The control group received a new high quality visco-elastic foam mattress covered with a cotton sheet. The intervention group received the same new high quality viscoelastic foam mattress, in combination with the newly developed multilayer system. The reason for use of the same (new) basic visco-elastic foam mattress in both groups was the intention to uniform the circumstances in the control homes. Research nurses followed the participating residents for 12 weeks. The outcome parameter was the development of PUs.

\section{Results:}

206 residents participated in the study. Both groups were comparable on patient characteristics (e.g. age, Braden score, care dependency, incontinence). In the control group $5 \%$ of the residents developed a category 2, 3 or 4 PU and in the intervention group $9 \%$ (a statistically not significant difference).

\section{Conclusion:}

This study shows that the multilayer system (MOS, barrier mattress cover \& STS), when used as an integral system and in combination with a visco-elastic foam mattress, has no added value over the visco-elastic foam mattress/cotton sheet. 
This study was possible by an unrestricted grant of Sense Textile, s' Hertogenbosch Netherlands. Design and execution of the study and the statistical analyses were done under supervision of Maastricht University. 


\section{Introduction}

In nursing homes, pressure ulcers (PUs) result in suffering and a substantial loss in quality of life of the residents involved. ${ }^{1,2}$ Next to this, PUs are associated with considerable extra health care costs., ${ }^{3,4,5,6}$

Incidence measurements in various health care settings are an indicator for the relevance of PUs. Vanderwee et al. found an incidence rate of PUs category 2-4 of $6.8 \%$ in hospital wards, during an observation period of 16 days. ${ }^{7}$ Meesterberends et al reported an incidence of cat 2-4 PUs of $22 \%$ and $7.8 \%$ in respectively Dutch and German nursing homes, for newly admitted residents that were followed longitudinally for a period of 12 weeks. ${ }^{8}$ De Souza found an incidence of $11.7 \%$ cat 2 PUs over a period of 90 days in long care facilities. ${ }^{9}$ In addition, most PUs in nursing home residents occur in the sacral region and at the heels, and many of them show a very long healing period. ${ }^{10,11,12}$ These figures clearly show that PUs are still a highly relevant problem, especially in nursing homes.

Pressure has long been considered as the only important extrinsic factor causing PUs. However, since 1985, also shear and friction have been reported to play a role in PU etiology. ${ }^{13}$

Recent and ongoing research has revealed however that also the phenomenon of the skin microclimate has a significant impact in the complex etiologic pathophysiology of PUs as well. ${ }^{14}$ These four causal factors are subsequently addressed in more detail.

Pressure is defined as the amount of force/load applied perpendicular per unit surface area of application. A force applied over a small area will produce a greater pressure than the same force over a larger area. When forces are oriented or exerted parallel to the skin they can be labelled as shearing or shear forces. Shearing is the mechanical force that is oriented parallel to the skin and that damages deep tissues including muscle tissue. Tissues attached to the bone are pulled in one direction while surface tissues remain in place. Shearing in bed most commonly occurs when the head of the bed is elevated and the resident slides downward.

Friction is the mechanical force of two surfaces (e.g. skin versus bed interface/chair interface) moving across each other, leading to tissue damage including blisters and abrasions. Residents who cannot lift themselves during repositioning and transferring are at high risk for friction injuries. Friction forces in bed are most common when residents are turned or pulled up in bed. ${ }^{14}$ 
Additionally, the phenomenon of skin interface microclimate has gained growing interest recently. Microclimate refers to skin temperature and moisture conditions at the skin-support surface interface. ${ }^{15}$ The maintenance of a balanced microclimate is considered to be a key modifier of the ability of the skin and underlying soft tissue to withstand prolonged stress. ${ }^{16}$

Recently PUs are divided into friction ulcers that develop at the surface of the skin and primarily are caused by friction, shear and trauma (category 1 and 2 PUs) and ulcers starting in deep tissue, caused by perpendicular forces and shear strength (category 3 and 4 PUs). ${ }^{14}$ Disturbances of the microclimate additionally do worsen both PU risk and PU status.

It will be clear that prevention of PUs in nursing home residents is of utmost importance. Next to paying attention to the health status of the residents, normally the first step in PU prevention involves the use of pressure reducing systems in bed and (wheel)chair. For sacral ulcers this means for instance taking care of redistributing pressure forces over a larger part of the body by using pressure relieving systems (mattresses and cushions) and shortening the period of pressure at one spot by applying repositioning. ${ }^{17,18}$ The current international guidelines for PU prevention recommend the use of a visco-elastic foam mattress with repositioning by schedule (every 3 hours by day and 4 hours by night) as a first step measure in this respect. ${ }^{19}$ Prevention of heel ulcers is normally done with redistribution surfaces or offloading devices such as heel protection devices or pillows under the calf, aiming to reach zero pressure at the heel bone. ${ }^{19}$

It is still unclear what the best methods are for realizing a good microclimate. An insufficient microclimate involves a climate at the skin-support surface interface that is characterized by a temperature that is too high or too low, and a humidity of the skin surface that is too high or too low. ${ }^{15}$ It has been proven that a rise in temperature of the skin results in about a $10 \%$ rise in cell metabolism, which in turn can lead to damage of the tissue in case there is blood flow reduction due to pressure on the skin. ${ }^{20,21}$ Increased skin moisture like for example perspiration or incontinence, influences the microclimate negatively as well. Excessive moisture (perspiration, urine or faeces) can weaken the skin surface considerably. ${ }^{22}$ This may result in a higher skin coefficient of friction (COF), leading to more damage due to friction and shear stress. ${ }^{14}$ Contrary to moist skin also a very dry skin can be a problem. A dryer, and locally rougher skin can lead to higher friction on a support surface (sticking effect). A dry skin, often occurring in frail elderly, has reduced lipid levels (necessary for cohesion of epidermal cells), water content, tensile strength and 
adhesion between epidermis and dermis. These factors lead to a higher vulnerability of the skin. ${ }^{23,24}$ Measurable microclimate parameters for resident support interfaces, sheets and mattresses are: water vapour resistance, resulting humidity and temperature after water absorption, and dry heat flux. ${ }^{25}$ Yusuf et al. measured in their study the resident support interface ability to cool down after heating (dry heat flux) and its ability to transfer water vapour (water vapour resistance or Ret value). ${ }^{26}$ Their conclusion was that increasing skin temperature can be used as a quantitative measurement to predict the development of pressure ulcers and superficial skin changes and to evaluate support surface capability against negative microclimate factors. Gefen developed a model that quantitatively demonstrated that a rise in skin temperature, ambient temperature, relative humidity and/or the pressure delivered to the skin from the support, as well as decreased permeability of the materials in contact with the skin or in close proximity to the skin, all raise the risk for superficial PUs. ${ }^{27}$ Lachenbruch et al. using laser Doppler flowmetry studied the effects of a higher skin temperature, pressure and shear stress on the superficial skin layer. The results suggest that managing skin pressure and temperature may reduce the risk of ischemia/PU development. ${ }^{28,29}$

The skin is permanently exposed to contact with fabrics during the activities of everyday life. Physical contact and friction at the skin-textile interface may also play an important role in the onset of mechanical skin irritations and skin injuries such as abrasions, blisters and PUs. ${ }^{30,31}$ Particularly, in bedridden persons, friction and moisture at the skin-textile interface are often associated with the feeling of discomfort. ${ }^{32}$ A high humidity can result in a high COF, while adequate regulation of moisture may result in a lower COF, possibly lowering the risk of development of a pressure ulcer. ${ }^{33} \mathrm{New}$ textile materials based on this knowledge may be helpful to reduce the development of PUs. Rotaru et al. did friction measurements under dry and wet conditions and found a 50\% reduction on a new prototype bed sheet (50\% cotton, $50 \%$ polyester) with low friction and beneficial water transport properties. ${ }^{34}$

It may be interesting to develop new devices which interfere with pressure forces, shear stress and regulation of the microclimate.

Recently, a new integral multilayer system (total thickness $13 \mathrm{~mm}$ ) was developed (Bedcare $^{\circledR}$ ), consisting of 3 separate layers each with an independent function: ${ }^{35}$

1. a 3-dimensional pressure relieving spacer fabric used as a supplement to the mattress, the Mini Overlay System (thickness $9.5 \mathrm{~mm}$ (MOS), placed on top of a normal basal mattress with the goal to assist in further reduction of pressure forces; 
2. a textile based mattress cover $(0.5 \mathrm{~mm})$ to protect both the regular basal mattress as well as the mini mattress layer against contamination and damage and 3 . a 3-dimensional knitted spacer fabric used to replace a normal bed sheet, the Stay and Transfer Sheet or STS (thickness $3 \mathrm{~mm}$ ), this is then the actual resident interface, it has a low coefficient of friction to diminish shear stresses and a knitted method that helps regulate the microclimate by vapour uptake, fluid dispersion, evaporation and ventilation.

Figure 1. Bedcare system

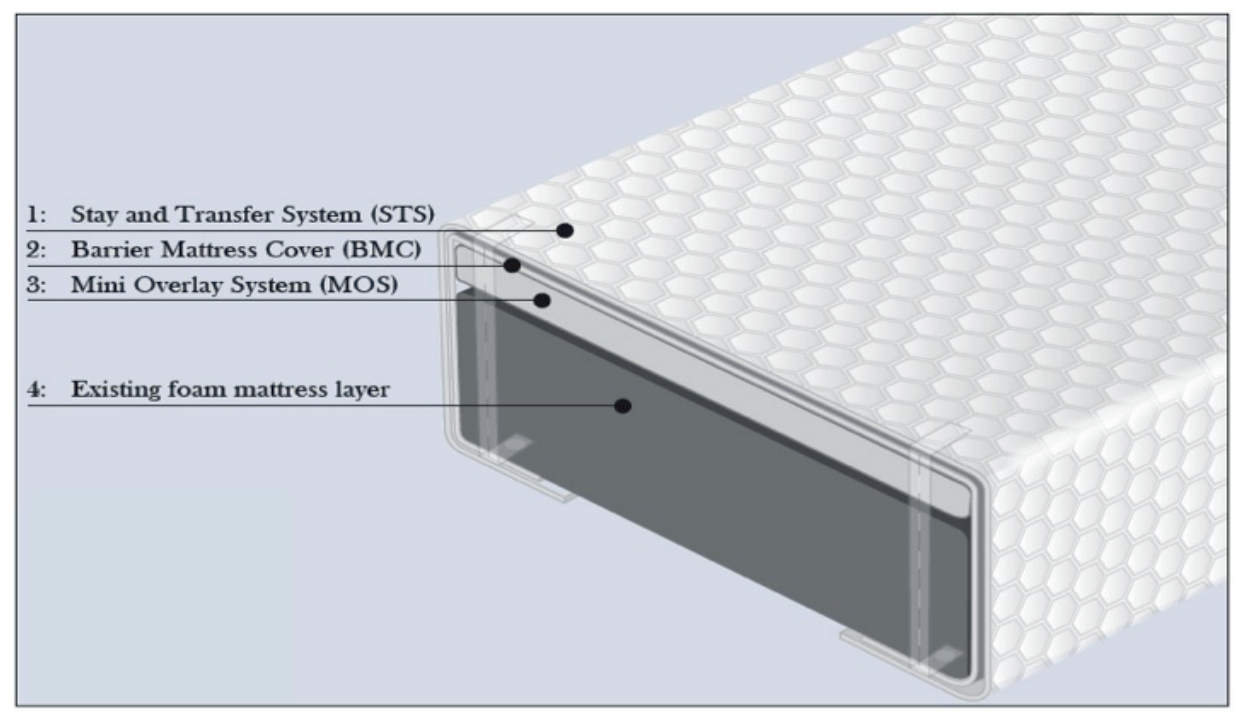

The 3-layers of the STS provide the following:

- a top layer made of a low friction very fine knitted textile fabric based on fine and smooth synthetic fibres, leading to a static and dynamic COF of 0.3 (dry sheet) and static COF 0.3 (wet sheet) in X and Y direction. These are very low COF values compared to for instance standard cotton sheets which show a static and dynamic COF of 0.4 (dry sheet) and 0.6 (wet sheet); this layer is in direct contact with the resident.

- a 3-dimensional intermediate layer with bendable pile yarns which is able to transport fluid (perspiration, urine) away from the top surface into an area where it is more easily evaporated in order to lower the humidity and the temperature of the skin interface and

- a bottom layer which is a very fine knitted textile fabric, less permeable, based on fine and smooth synthetic fibres. The complete STS resident interface results 
in a lower friction force during movements of the resident him or herself or during repositioning and transfer handlings of the nurses.

It is hypothesised that by combining MOS, mattress cover and STS into an integral multilayer Bedcare system and using it in conjunction with a visco-elastic mattress, additional reduction of pressure (MOS), shear stress (STS), and optimisation of the microclimate (STS) will be achieved. Ultimately this should lead to a lower PU incidence than the expected incidence by using a visco-elastic foam mattress/cotton sheet alone.

Therefore, the intention of this study was to explore the effect of the total multilayer system $\left(\right.$ Bedcare $\left.^{\circledR}\right)$ on PU incidence in nursing home residents.

The research question addressed was:

What is the effect of the multilayer system (Bedcare ${ }^{\circledR}$ ) when used in conjunction with a regular and new high quality visco-elastic foam mattress (standard according to the international guidelines) on PU incidence in nursing home residents?

\section{Methods}

\section{Study design}

This study was set up as a multicenter, prospective, controlled randomized clinical trial in 21 voluntarily participating Dutch nursing homes. The total study period was 12 weeks.

\section{Study population and control versus intervention}

All residents at medium/high risk (Braden score $<16$ ) of PU were asked to participate and observed for a period of 12 weeks. The inclusion criteria were: age $>60$ years, life expectancy $>3$ months, informed consent. The exclusion criteria were: no pressure ulcer in the last 3 months, participation in a comparable trial, or a physical and/or mental condition which could interfere with participation (sepsis, immune disease, palliative status).

After informed consent, randomization into two groups was performed by using the Castor randomization software. ${ }^{36}$ To detect a clinically relevant reduction of the incidence of pressure ulcers of 30\% with a statistical safeguard of $\alpha 0.05$ and a power of $0.80,220$ residents were needed. 
In order to uniform standard care, the control group received a new high quality visco-elastic foam mattress, covered with a normal cotton bed sheet while the intervention group received the same new high quality visco-elastic foam mattress together with the new multilayer system $\left(\right.$ Bedcare $\left.^{\circledR}\right)$ instead of the normal cotton bed sheet. When out of bed, all residents were sitting on a preventive air pillow according to the standard preventive PU protocol, in line with the international guidelines. For the rest, both groups received standard care by protocol.

The Medical Ethical Committee Brabant, Tilburg, The Netherlands approved the study (number NL48831.028.14/P1414). In addition the study was also recorded in the Dutch trial register (number 4557).

\section{Data collection}

Four research nurses were responsible for collecting the data. After inclusion, the following data were recorded at the start of the study: age, gender, main diagnosis, co-morbidity, medication, skin condition, incontinence yes/no, Body Mass Index (BMI), type of PU preventive measures used (e.g. cushions in (wheel)chair, offloading devices for the heels, repositioning, transfers in/out bed).

To assess the risk of pressure ulcers the Braden scale was used. This is a scale with a proved validity and reliability. ${ }^{37,38}$ The scale measures PU risk, making scores from 1 to 4 regarding mental and physical condition, activity, mobility and incontinence. The maximum score is 23 (no risk at all) and the minimum score is 7. A score of 19 or lower indicates a risk for PUs; (a score of 13-17 represents medium risk and a score of 7-12 high risk). ${ }^{37}$

Care dependency at start was measured by the MDS-ADL (RAI, section physical functions). ${ }^{39}$ The MDS-ADL measures bathing, self-care, getting dressed, mobility, transfers, toilet use, bed mobility and eating; each item of the scale is assessed on a 6-point Likert scale and the total score ranges from 0 to 72; the higher the score, the more care dependent.

In the follow-up period weekly controls were done related to the following aspects: inspection of skin condition, changes in use of PU preventive measures and possible adverse events. Data collection was weekly controlled by an independent research nurse. 


\section{Outcome parameters}

The primary outcome parameter was the development of a category 2, 3 or $4 \mathrm{PU}$ according to the NPUAP/EPUAP/PPPIA guideline $2014 .{ }^{19}$ Category 1 PUs were excluded because of the known insecurity of the diagnosis. The staging of the PUs was done by the research nurses.

\section{Data analysis}

Statistical analyses were done by using IBM SPSS Statistics version 23 (SPSS Inc., Chicago, IL USA). Differences between the control and intervention group were tested with the student $t$ test for continuous variables and a chi-quadrate test for categorical variables.

\section{Results}

In total, 215 residents signed informed consent. Nine residents were not able to participate, because of initial organisational problems in some participating nursing homes. 206 residents could be included in the study, 103 in each group. In total, 165 residents completed the full period. 17 Residents stopped participating on their own initiative or because of hospitalisation ( 9 in control group and 8 in intervention group). 24 residents died, 12 in each group, in all cases not related to the study. The nursing staff and the research nurses did not report any adverse events during the study. Table 1 shows the characteristics of the participating residents.

Table 1. Characteristics of all residents $(n=206)$

\begin{tabular}{|l|c|c|c|}
\hline Characteristics at start study & $\begin{array}{c}\text { Control Group } \\
\mathbf{N = 1 0 3}\end{array}$ & $\begin{array}{c}\text { Intervention Group } \\
\mathbf{N = 1 0 3}\end{array}$ & P-value \\
\hline Age in years & 81.7 & 83.1 & 0.285 \\
\hline Gender (females) & 69.9 & 71.8 & 0.759 \\
\hline Dementia & 54 & 50 & 0.577 \\
\hline CVA & 29 & 27 & 0.754 \\
\hline Braden start score 6-15 & 13.3 & 13.1 & 0.458 \\
\hline MDS-ADL start & 46.9 & 45.9 & 0.517 \\
\hline BMI start & 24.6 & 25.0 & 0.567 \\
\hline Incontinence (\%) & 94.1 & 94.2 & 0.381 \\
\hline
\end{tabular}


Most residents in both groups were above 80 years of age, female and very care dependent. The two most important main diagnoses (CVA and dementia) were representative for the overall nursing home population. There were no statistically relevant differences between the control and the intervention group.

The development of pressure ulcers in the control and intervention group is shown in table 2. In the control group 5 residents developed a PU and in the intervention group 9 residents. This difference was not statistically significant $(\mathrm{P}=0.180)$.

Table 2. Incidence of pressure ulcers (cat. 2 and higher) per condition.

\begin{tabular}{|c|c|c|c|c|c|c|c|}
\hline & & \multicolumn{3}{|c|}{$\begin{array}{l}\text { Control group } \\
\text { (Visco-elastic foam mattress + cotton } \\
\text { sheet) } \\
n=103\end{array}$} & \multicolumn{3}{|c|}{$\begin{array}{l}\text { Intervention group } \\
\text { (Visco-elastic foam mattress with } \\
\text { Bedcare system) } \\
n=103\end{array}$} \\
\hline \multicolumn{2}{|c|}{ Pressure ulcers } & \multicolumn{3}{|c|}{ Location } & \multicolumn{3}{|c|}{ Location } \\
\hline & & sacral & heel & other & sacral & heel & other \\
\hline \multirow[t]{3}{*}{ Category } & 2 & 1 & 0 & 2 & 3 & 3 & 1 \\
\hline & 3 & 0 & 2 & 0 & 0 & 1 & 1 \\
\hline & 4 & 0 & 0 & 0 & 0 & 0 & 0 \\
\hline
\end{tabular}

\section{Discussion}

The results of this study show that the initial hypothesis that applying the integral multilayer system on a visco-elastic mattress should lead to a lower PU incidence than the expected incidence by using a visco-elastic foam mattress/cotton sheet alone, is not confirmed. The control group showed a PU incidence $4.9 \%$ and the intervention group $8.7 \%$. The low PU incidence in the control group confirms the primary PU prevention strategy of using an adequate visco-elastic foam mattress as stated in the international EPUAP/NPUAP/PPPIA guideline. ${ }^{19}$ The question arises why the expected positive additional effect of the new multilayer system was not observed in this study. To explore possible reasons for this, additional laboratory pressure tests were performed by the Institut für Textil- und Verfarenstechnik Denkendorf (Germany), on the used visco-elastic foam mattress and also on the same mattress in combination with the pressure relieving part of the multilayer system (MOS). ${ }^{40}$ Figure 2 shows the pressure test results. 
Figure 2. Pressure test results post study period

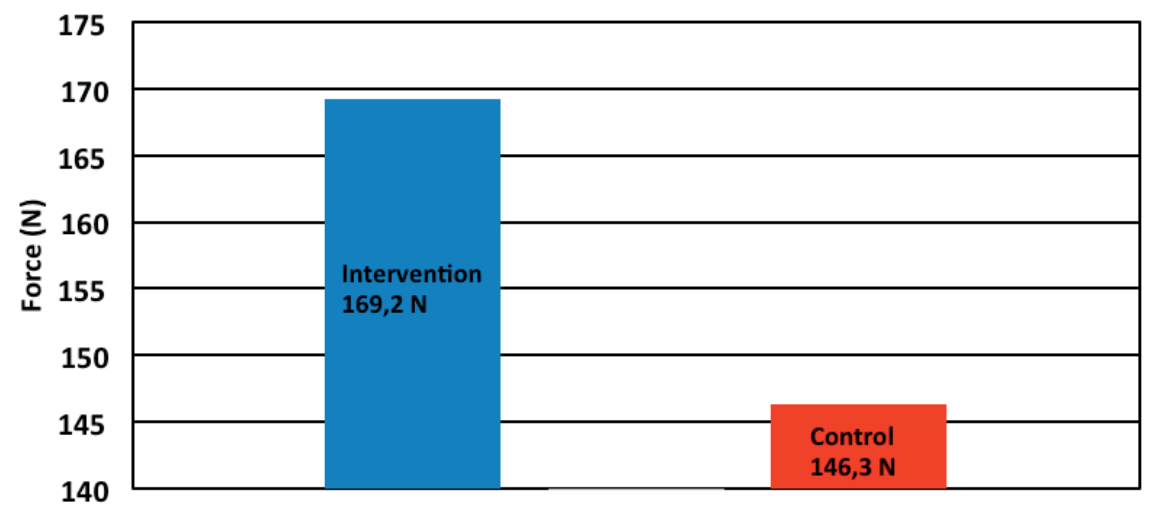

Results of tests showing the required pressure to achieve the same indent: viscoelastic foam mattress (control group, red column) vs visco-elastic foam mattress incl. MOS (intervention group, blue column); force in Newton (N)

This graph shows how many Newtons are required to achieve the same surface contact indentation (support surface area). The more the body is indented in a support surface the larger the contact area for pressure distribution of the body weight (=immersion and envelopment). These measurements show that in the intervention group the patient would not indent the mattress as much as a patient would on a control mattress. The intervention mattress would provide less indentation and therefore less surface area resulting in higher pressure load being distributed over a lower area. If one would calculate for example the weight of a foot, and the surface area of a heel, or bony proximities such as ankles or hips, one might conclude that the results of load distribution here could be at least a factor $2.2 \mathrm{x}$ higher than on the control mattress. Therefore, the surprising results of this test may be the explanation of the withdrawal of the original hypothesis.

The possible, separate additional benefits of the STS part of the multilayer system related to reduction of the shear stress, regulation of the microclimate, patient 
comfort and nursing feasibility could unfortunately not be assessed in this study and should be explored in future studies.

\section{Strength and limitations}

Compared to other studies, this study is one of the largest randomised controlled trials on the PU preventive effect of mattresses/mattress overlays ever performed in nursing homes. Moreover special attention has been paid to standardising the basic mattress in both groups. Because it was necessary to include more than 200 residents at medium/high risk, 21 nursing homes were ultimately needed. Differences in the use of other PU preventive measures (e.g. skin care, nutritional status, fluid intake, repositioning, offloading of heels in control group) may possibly have influenced the results, which can be considered as a limitation.

\section{Conclusion}

This study shows that the multilayer system (MOS, barrier mattress cover \& STS), when used as an integral system and in combination with a visco-elastic foam mattress, has no added value over the visco-elastic foam mattress/cotton sheet. 


\section{REFERENCES}

1. Gorecki C, Nixon J, Madill A, Firth J, Brown JM. What influences the impact of pressure ulcers on health-related quality of life? A qualitative resident-focused exploration of contributory factors. Journal of Tissue Viability Volume 21, Issue 1, February 2012, Pages 3-12

2. Gorecki C, Brown JM, Nelson EA, Briggs M, Schoonhoven L, Dealy C et al. Impact of pressure ulcers on quality of life in older residents: a systematic review. Journal of the American Geriatric Sociaty 57:11751183,2009

3. Demarré L, Verhaeghe S, Annemans L, van Hecke A, Grypdonck M, Beeckman D. The cost of pressure ulcer prevention and treatment in hospitals and nursing homes in Flanders: a cost-of-illness study. Int J. Nurs. Stud. (2015), http://dx.dol.org/10.1016/j.ijnur-stu.2015.03.005

4. Pham Ba, Stern A, Chen W, Sander B, John-Baptiste A, Thein H et al. Preventing pressure ulcers in long-term care. A cost-effectiveness analysis. Arch Intern Med 2011;171(20):1839-1847

5. Dealey C, Posnett J, Walker A. The costs of pressure ulcers in the United Kingdom. Journal of Wound Care. 2012;21(6):261-266

6. Brem H, Maggi J, Nierman D, Rolnitzky L, Bell D, Rennert R et al. High costs of stage IV pressure ulcers. American J Surg. 2010;200(4):473-477

7. Vanderwee K, Grypdonck M, Defloor T. Non-blancheble erythema as an indicator for the need for pressure ulcer prevention: a randomised controlled trial. J Clin Nurs 2007 Feb;16(2):325-35

8. Meesterberends E, Halfens RJG, Spreeuwenberg MD, Ambergen TAW, Neyens JCL, Schols JMGA. Do residents in Dutch nursing homes have more pressure ulcers than residents in German nursing homes? A prospective multicentre cohort study. JAMDA 2013;14:605-610 doi: 10.1016/j.jamda.2013.03.005.

9. De Souza DM, De Gouveia Santos VL. Incidence of pressure ulcers in the institutionalized elderly. Journal of Wound, Ostomy and Continence Nursing 2010;37:272-276

10. Halfens RJG, J.M.M. Meijers, Meesterberends E, Neyens JCL, Rondas AALM, Rijcken S, Wolters S, Schols JMGA. National Prevalence Measurement of Care Problems 2014. Caphri school for public health and primary care, Maastricht, Netherlands 2014.

11. Berlowitz DR, Brandeis GH, Anderson J, Brand HK. Predictors of pressure ulcer healing among long-term care residents. J. AM. Geriatr. Soc.1997;45:30-34

12. Shannon MM. A retrospective descriptive study of nursing home residents with heel escars or blisters. Ostomy Wound Management vol 59, issue1, Jan 2013

13. Bennett L, Lee BY. Pressure versus shear in pressure causation. Chronic ulcers in the skin 1985;39-56. New York: McGraw-Hill

14. Orsted HL, Ohura T, Harding K. International review. Pressure ulcer prevention: Pressure, shear, friction and microclimate in context. A consensus document. Londen Wounds International, 2010

15. Clark M, Romanelli M, Reger SI, Ranganathan VK, Black J, Dealey. Microclimate in context. International review. Pressure, shear, friction and microclimate in context. A consensus document. London: Wounds international, 2010

16. Reger SI, Ranganathan VK, Orsted HL, Ohura T, Gefen A. Shear and friction in context. International review. Pressure ulcer prevention: pressure, shear, friction and microclimate in context. A consensus document. Londen Wounds International, 2010.

17. Roaf R. The causation of preventing bedsores. J Tissue Viability 2006;16(2):6-8 
18. BrienzaDM, Geyer MJ. Using support surfaces to manage tissue integrity. Adv Skin Wound Care 2005;18:151-57

19. NPUAP/EPUAP/PPPIA. Prevention and treatment of pressure ulcers: a quick Reference guide 2014. http:// www.internationalguideline.com/static/pdfs/NPUAP-EPUAP-PPPIA-PUQuickReferenceGuide.pdf

20. Rapp MP, Bergstrom N, Padhye NS. Contribution of skin temperature regularity to the risk of developing pressure ulcers in nursing facility residents. Adv Skin Wound Care 2009;22(11):506-13

21. Sae-Sia W, Wipke-Tevis DD, Williams DA. Elevated sacral skin temperature (Ts): a risk factor for pressure ulcer development in hospitalized neurologically impaired Thai residents. Appl Nurs Res 2005;18:29-35

22. Clark M. The aetiology of superficial sacral pressure sores. In: Leaper D, Cherry G, Dealey C, Lawrence J, Turner T, editors. Proceedings of the 6th European Conference on Advances in Wound Management. Amsterdam: McMillan Press; 1996. p. 167-70

23. Mayrovitz HN, Sims N. Biophysical effects of water and synthetic urine on skin. Adv Skin Wound Care $2001 ; 14(6): 302-8$

24. Egawa M, Oguri M, Kuwahara T, Takahashi M. Effect of exposure of human skin to a dry environment. Skin Res Technol 2002; 8(4): 212-18

25. Williamson R, Lachenbruch C, VanGilder C. A Laboratory Study Examining the Impact of Linen Use on Low-air-loss Support Surface Heat and Water Vapor Transmission Rates. Ostomy Wound management 2015;61(2):16-25

26. Yusuf S, Okuwa M, Shigeta Y, Dai M, Iuchi T, Rahman S, Usman A, Kasim S, Sugama J, Nakatani T, Sanada H. Microclimate and development of pressure ulcers and superficial skin changes. Int Wound J 2015; 12:40-46

27. Gefen A. How do microclimate factors affect the risk for superficial pressure ulcers: A mathematical modelling study Journal of Tissue Viability (2011) 20, 81-88

28. Lachenbruch C, Tzen Yi-Ting, Brienza D, Karg PE, Lachenbruch PA. Relative contributions of interface pressure, shear stress and temperature on ischemic-induced skin-reactive hyperemia in healthy volunteers: a repeated measure laboratory study. Ostomy Wound Management 2015;61(2):16-25

29. Lachenbruch C, Tzen Yi-Ting, Brienza D, Karg PE, Lachenbruch PA. Relative contributions of interface pressure, shear stress and temperature on tissue ischemia: a cross-sectional pilot study. Ostomy Wound Management 2013;59(3):25-34

30. Gerhardt LC, Strassle V, Lenz A, Spencer ND, Derler S. Influence of epidermal hydration on the friction of human skin against textiles. J.R.Soc.Interface (2008)5,1317-1328 doi.10.1098/rsif.2008.0034

31. Derler S, Rao A, Balleistreri P, Huber R, Scheel-Sailer A, Rossi RM. Medical textiles with low friction for decubitus prevention. Tribology International 46 (2012) 208-214 doi:10.1016/j.triboint.2011.03.011

32. Gerhardt LC, Strassle V, Lenz A, Spencer ND, Derler S. Influence of epidermal hydration on the friction of human skin against textiles. J.R.Soc.Interface (2008)5,1317-1328 doi.10.1098/rsif.2008.0034

33. Derler S, Gerhardt LC. Tribology of skin: review and analysis of experimental results for the friction coefficient of human skin. Tribol Lett (2012) 45:1-27 doi 10.1007/s11249-011-9854-y

34. Rotaru GM, Pille D, Lehmeier FK, Stämpfi R, Scheel-Sailer A, Rossi RM, Derler S. Friction between human skin and medical textiles for decubitus prevention. Tribology International 65 (2013)91-96 http://dx.doi. org/10.1016/j.triboint.2013.02.005

35. Institut für Textil- und Verfarenstechnik Denkendorf. Unsere auftrags-NR.:E-0230-TT-15, 07-10-2015. Available at: www.sensetextile.com 
36. Castor Software V1.44. http://support.mionix.net/entries/83608925-CASTOR-Software-V1-44

37. Braden D. Calculating the risk: reflections on the Braden Scale. Advances in Wound Care 1989;9:38-43

38. Braden BJ, Bergstrom N. Predictive validity of the Braden scale for pressure sore risk in a nursing home. Research in Nursing \& Health 1994;17:459-470

39. Revised Long-Term Care Facility Resident Assessment Instrument User's Manual Version 2.0 December 2002. http://www.interrai.org/scales.html

40. Institut für Textil- und Verfarenstechnik Denkendorf. Untersuchungsbericht NR.E-0076-TT-16, 11-042016. Available at: www.sensetextile.com 
o

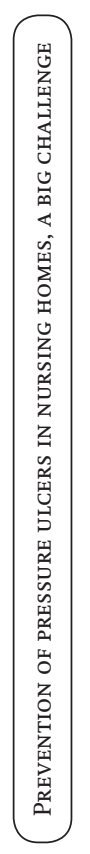


Chapter 7

GENERAL DISCUSSION 
O

100

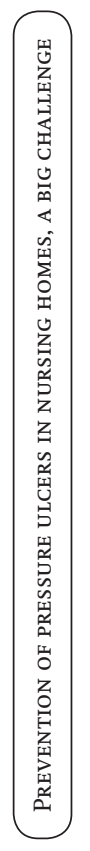




\section{Introduction}

The studies of this thesis were conducted in Dutch nursing homes. Firstly, they have provided more insight in the use of the recommended guideline of measures to prevent pressure ulcers (PU). Secondly, the PU preventive effect of pressure relieving foam mattresses with or without add-ons has been explored. Finally, a new 3-step PU preventive protocol has been developed and tested.

The specific research questions of this thesis were:

1. To what extent did Dutch nursing homes apply PU preventive measures, as recommended in valid (inter)national guidelines, during the period 2005-2014?

2. What is the clinical efficacy of combining a standard $15 \mathrm{~cm}$ cold foam mattress with a static air overlay mattress versus a cold foam mattress alone in preventing pressure ulcers in nursing home residents?

3. What is the clinical efficacy of a combination of a standard $15 \mathrm{~cm}$ visco-elastic foam mattress with a static air overlay mattress versus a standard $15 \mathrm{~cm}$ viscoelastic foam mattress alone in preventing pressure ulcers in nursing home residents?

4. What is the effect of implementing a 3-step PU prevention protocol on PU prevalence in the Avoord nursing homes (Etten-Leur, The Netherlands) over the period 2005-2011?

5. What is the effect of a multilayer skin interface microclimate regulating system, used in conjunction with a regular, visco-elastic foam mattress on PU incidence in nursing home residents?

This chapter provides an overview of the main findings described in the previous chapters of this thesis. Thereafter, theoretical and methodological considerations will be discussed. Finally, recommendations for daily practice and further research will be presented.

\section{Main findings}

The studies of this thesis showed the following main findings:

- In the participating Dutch nursing homes, over the years, none of the guideline recommended measures for prevention of PU were fully incorporated in the daily care for residents at risk of PU development.

- The use of a static air overlay mattress on top of a cold foam mattress tended to result in a lesser (non-significant $\mathrm{p}=0.088$ ) development of PUs and need of repositioning on the overlay mattress when compared to the use of only a cold foam mattress. 
- The use of a static air overlay mattress on top of a visco-elastic foam mattress tended to result also in a lesser (non-significant $\mathrm{p}=0.087$ ) development of PUs and need of repositioning on the overlay mattress when compared to the use of only a visco-elastic foam mattress.

- Implementation of a newly developed 3-step PU prevention protocol in a large Dutch nursing home organization (with six nursing homes) resulted in a fast decrease of category 2, 3 and 4 PU prevalence in one year. This remained stable during a six year period.

- The hypothesis that application of a microclimate influencing multilayer system on a visco-elastic foam mattress should lead to a lower PU incidence when compared to the use of only a visco-elastic foam mattress, was not confirmed.

\section{Theoretical considerations}

In nursing homes, a large number of residents develop a PU during their stay. ${ }^{1,2,3}$ PUs may result in considerable suffering, pain, social isolation, increased morbidity and higher mortality rates. ${ }^{4}$ Despite the availability of adequate evidence based guidelines, the incidence and prevalence of PUs in nursing homes in western countries, including the Netherlands, stays high. Next to this, pressure ulcers are associated with considerable extra health care costs, because of the need of specialist nursing and medical care. ${ }^{5}$ That's why PU prevention remains of utmost importance, especially in the group of frail nursing home residents. Factors, which may lead to better PU care involve adequate dissemination and implementation of evidence based PU preventive guidelines. Subsequently with application of relevant PU preventive measures, including PU preventive mattresses/cushions, repositioning, skin care, nutrition and underlying disease management. The application of relevant PU preventive measures in Dutch nursing homes was researched in this thesis.

\section{Compliance to guideline recommendations on PU prevention}

A secondary analysis of data from the Dutch National Prevalence Measurement of Care problems (LPZ) over the period 2005-2014 showed that none of the in (inter) national guidelines recommended PU preventive measures were fully incorporated in the basic care for nursing home residents at risk of PU development. ${ }^{6,8,8}$

It is difficult to compare the results of this study with other studies in nursing homes, because most other articles only report over a one year period. ${ }^{10,11}$ Nevertheless, most of these articles also show the same insufficient use of PU preventive measures. 
There may be several reasons for insufficient implementation of guideline recommended PU preventive measures in nursing homes. One reason could be that the dissemination of the guidelines has not been done extensive enough and therefore the awareness of and familiarity with the guidelines is too low. Meesterberends et al. however, showed that most nursing homes in several European countries including The Netherlands, did have an institutional pressure ulcer preventive guideline and that the dissemination of the guidelines seemed to be successful. ${ }^{12}$ But having a guideline does not mean that the recommendations derived from it will be automatically used in daily practice. Another conclusion of the study of Meesterberends et al. was that barriers to apply guideline recommendations in daily practice were mostly related to a lack of (qualified) personnel and knowledge and also because of poor communication. Demarré et al. showed that only 6-9\% of the residents in nursing homes received a fully compliant prevention. ${ }^{13}$ According to the authors the main reasons were lack of knowledge of preventive advice and a less positive attitude for prevention strategies. The same results have been found in Scandinavian countries, Iceland and Ireland. ${ }^{10,11}$

Demarré et al. also reported that assessing the knowledge and attitudes of staff members may provide information that can assist in the development of organization-specific interventions, which may result in better implementation of PU preventive care. ${ }^{13}$

The National Pressure Ulcer Advisory Panel, the European Pressure Ulcer Advisory Panel and the Pan Pacific Pressure Injury Alliance developed a guideline on the prevention and treatment of pressure ulcers, which was published in $2014 .^{14}$

This guideline gives also several advices for a better guideline implementation in daily practice, including the following: 1) assess barriers and facilitators for guideline implementation like knowledge and attitude of professional staff; 2) enforce the use of validated PU risk assessment tools; 3) take care of availability of enough PU preventive materials (mattresses and cushions); 4) conduct regular evaluations of local performance in PU prevention and consider optimizing work procedures. ${ }^{21}$

All these studies show clearly that the implementation of guidelines is challenging.

To overcome the implementation barriers mentioned above and to encourage a better implementation of PU guidelines active implementation strategies have shown to be more effective and should be advised above passive strategies. ${ }^{15,16,17,18,19,20}$ To execute this active implementation strategy, the approach should be very 
structured, especially in the nursing home setting where the general educational level of the nursing staff is low. Next to education, continuous feedback and reminder systems are essential, achievable via direct coaching on the job by professionals with both expertise on PU care and implementation issues. Structural support of the management should be there as well and also the availability of relevant PU preventive materials. Development and application of a computerised incidence register for PUs with monthly reports and also periodic PU prevalence measurement may be essential to realise enough and continuous commitment within the nursing staff. ${ }^{21}$ Finally, another reason for the insufficient implementation of running guidelines for PU prevention may be that most guidelines are to comprehensive and do not provide an integral schedule of simple and practical advices for everyday use.

104 Therefore, in chapter 5 of this thesis, we successfully introduced a simple 3- step model, which is easy to implement and to be followed in daily practice.

\section{PU preventive effect of high quality foam and add-ons}

The results of the first RCT about the preventive effects of a static air overlay mattress on top of a cold foam mattress, as presented in chapter 3 , showed that application of the static air overlay seems to provide a better PU prevention than using a cold foam mattress alone. In the intervention group of this RCT, repositioning as a measure for additional PU prevention was only used when there were signs of a developing PU. Contrary, in the control group, lying on the cold foam mattress alone, repositioning was done structurally by protocol. Despite the significantly lesser application of repositioning, PU incidence in the intervention group did not increase, supporting even further the efficacy of the static air mattress.

The same results were achieved in the second RCT, as presented in chapter 4, exploring the effect of an additional static air overlay on a visco-elastic foam mattress. Again the application of the static air overlay resulted in a non-significant lesser development of PUs and significantly lesser need of repositioning.

At the start of this $\mathrm{PhD}$ thesis trajectory, there were only a few articles available in literature, which compared the PU preventive effects of a standard foam mattress versus a static air overlay. Takala et al.(1996) analysed the PU preventive effect of a static air overlay over a period of 14 days in patients staying on an intensive care unit. $^{22}$ In the group patients on a static air overlay (21 patients) no PUs developed. Sanada et al. (2003) recruited patients on an acute care department and found a $4 \%$ incidence of category II PUs, when patients were lying on a double-layer air cell overlay versus $22 \%$ on a not further specified foam mattress. ${ }^{23}$ The article did not 
mention the study follow-up time. Both these studies were done in hospitals and no studies were published about nursing home residents. As we look at more recent studies and also at studies which compared other types of 'low tech' continuous low pressure mattresses, there are two studies available. Serraes et al. found in a cohort study in six nursing homes in Belgium a 5.0\% incidence of PU cat 2-4 development over a period of 30 days. ${ }^{24} \mathrm{~A}$ study of Vermette et al., who tested a static air overlay versus a Micro Static fluid Overlay (based on foam) for PU prevention in nursing home residents, also found a low PU incidence in the static air group (4\%) and of $11 \%$ in the Micro Static fluid Overlay group. ${ }^{25}$ Furthermore, until now, no other comparable studies have become available. ${ }^{26}$

Nowadays, the (inter)national guidelines also advise to look at other PU preventive aspects than pressure relieving and shear diminishing alone. Specific attention is paid to controlling microclimate. ${ }^{6,7,14}$ An insufficient microclimate involves a climate at the skin-support surface interface that is characterized by a temperature that is too high or too low, and a humidity of the skin surface which is too high or too low. ${ }^{27}$ A rise in temperature of the skin of one degree Celsius results in about a $10 \%$ rise in cell metabolism, which in turn can lead to damage of the tissue. ${ }^{28,29}$ Excessive moisture (perspiration, urine or faeces may weaken the skin surface considerably. ${ }^{27}$ This may result in a higher skin coefficient of friction (COF), leading to a higher susceptibility for damage because of friction and shear stress. ${ }^{30}$ Contrary to a moist skin, also a very dry skin can cause problems. A dryer, and locally rougher skin may lead to higher friction on a support surface. ${ }^{31,32}$ These findings have urged the development of skin interface microclimate regulating systems.

Therefore, in this thesis, in addition to the earlier two studies on foam mattresses with add-ons, we also explored the effect of using a multilayer skin interface microclimate regulating system on top of a visco-elastic foam mattress versus using a cotton sheet alone on top of the same type of mattress, on the incidence of pressure ulcers.The multilayer system was developed to reduce pressure, shear and friction and to regulate the microclimate at skin interface level. ${ }^{33,34}$ The study was set up as a multicenter, prospective, controlled randomized clinical trial over a study period of 12 weeks. The results showed, that the initial hypothesis, that applying this system on a visco-elastic mattress should lead to a lower PU incidence, was not confirmed. In the intervention group a PU incidence was found of $8.7 \%$ and in the control group a PU incidence was found of $4.9 \%$. The most probable explanation of this unexpected negative finding was the fact that all participating residents in both groups received a new visco-elastic foam mattress as basic mattress to make both the intervention group and control group comparable on the use of the baseline regular foam 
mattress.This study learned us additionally to pay more attention to the age and related PU preventive performance of the foam matresses used in daily PU care. This means, paying attention to the aspect of the garanty-aspect of the manufacturer that the memory effect of foam is only certain for a period of five years. This aspect has also been stated in the NPUAP-EPUAP-PPPIA guideline on prevention and treatment of PUs 2014 and involves that in regular PU care the foam matresses used for basic PU prevention should be replaced every 5 years. ${ }^{14}$

In addition, Vanderwee et al. studied the incidence of PU development by applying different types of mattresses, with a cotton sheet as interface and they found an incidence of category 2, 3 and 4 PUs of 15.3\% during use of a cotton sheet on a viscoelastic foam mattress. ${ }^{35}$ In the Cochrane review about pressure reducing systems from 2015, percentages between 15 and 25\% were also mentioned for older RCTs with visco-elastic foam. ${ }^{36}$ In the foam mattress studies of this Cochrane review however, in both the control and the intervention groups, rather old and not uniform foam mattresses were used. Compared to the results of these older studies, the use of the new multi-layer system in fact shows a better preventive result compared to the use of a cotton sheet as interface. However, it is to early to advise about the preventive use of the multilayer system tested in this thesis, also because the results of using the new visco-elastic foam mattress alone show such a good outcome.

\section{Repositioning YES or NO?}

In all PU guidelines there is a strict advice to use repositioning for all residents at risk of developing pressure ulcers. ${ }^{6,7.8 .9}$ The already long existing advice is that residents with reduced mobility and at risk of PUs must be repositioned in bed every 3-4 hours. A further recommendation is to use a $30^{\circ}$ tilted side lying position, if acceptable given the medical condition of the resident. ${ }^{37,38}$ However, the evidence to apply repositioning to prevent pressure ulcers is low and it is still unknown if particular positions or frequencies of repositioning reduce pressure ulcer development. ${ }^{39}$ Though theoretically it seems logical to reduce the time of deformation of tissue (epidermis or deeper layer) by pressure and shear by using repositioning, the authors of a recent Cochrane review on repositioning (April 2014) found only three studies which recruited 502 people. The evidence on supporting the use of repositioning to prevent pressure ulcers appeared to be low in volume and quality and we still do not know if particular positions or frequencies of repositioning reduce pressure ulcer development. In addition, none of the trials reported on pain or quality of life. That's why, the authors pointed to the lack of scientific evidence and clearly stated that further research about the best way of using repositioning is necessary. ${ }^{39}$ 
Furthermore, in daily practice of PU care, often a bad compliance is shown in the use of repositioning. This was shown again in our study on the application of PU preventive measures in chapter 2. Moore et al. (2011) found a low use of repositioning as well; it involved only $9 \%$ of elderly patients of Irish long term care facilities and no difference was found in the use of repositioning in patients with and without a PU. ${ }^{40}$ Breimeyer et al. (2013) found in Austrian nursing homes that about $40 \%$ of the patients at risk of a PU received repositioning. ${ }^{41}$ Furthermore, we know that the repositioning process is labour intensive and time consuming. In times of financial constraints for the long-term care sector in many countries, including The Netherlands, the question rises whether repositioning should be a standard measure for all patients at risk? This is even more appropriate, because we see a trend of decreasing prevalence of PU development in the Netherlands over the years (see chapter 2).

It is the reason why in the two RCTs of chapter 2 and 3 in both the control and intervention groups we deliberately chose to ignore the standard application of repositioning in all residents at risk of PUs and only to use repositioning when there were signs of a categorie II PU. Contrary to the advice in the current PU guidelines, the results of both RCTs showed that standard repositioning of all patients at risk was not necessary and this finding, therefore, can be considered as very interesting for daily PU care.

\section{Implementation and efficacy of a newly developed 3-step PU prevention protocol}

As we mentioned in the beginning of this chapter, the current(inter)national guidelines forprevention ofPUsmaybetoocomplextoexecuteintegrallyin dailyPUcare. Moreover, clearevidenceforsomerecommendationsislacking, e.g.theevidencefor repositioning. Following the findings of our studies in chapter 2-4 we developed a more simple and straightforward 3-step PU prevention protocol of which we hypothesized that it might already lead to a considerable reduction of PU development. This protocol was implemented in a large Dutch nursing home organization (Avoord, Etten-Leur, The Netherlands).

In the 3-step protocol the following steps are applied, subsequently:

- step1: all nursing home residents (including residents at risk of PUs) are lying on a standard visco-elastic foam mattress, without repositioning;

- step 2: patients who develop signs of a possible category 1 PU (non-blanchable redness) receive additionally a static air overlay on top of the visco-elastic foam mattress without repositioning; 
- step 3: patients, who still develop a PU (category 1 or more) get repositioning every 3 hours by daytime and every 4 hours by nighttime. The repositioning schedule for only these specific residents is still based on the advice of the current guidelines. ${ }^{6,7,8,9}$

The study, presented in chapter 5, aimed to explore the effect of implementing this 3-step PU prevention protocol on PU prevalence in the Avoord nursing homes (Etten-Leur, The Netherlands) over the period 2005-2011. The results showed that in the first year after implementation of this newly developed protocol the PU prevalence decreased enormously, from $8.7 \%$ to $0.5 \%$, and remained stable in the years thereafter, with a prevalence figure of around $2 \%$. Nationally, the annual LPZ data showed a small fluctuation in repositioning percentage between $10-17 \%$ in nursing homes, while the use of repositioning in the nursing homes of Avoord showed a decrease from 22\% in 2005 to $7 \%$ in 2006 and afterwards a trend of stabilization. Additionally, in Dutch nursing homes 11.7\% (9.7-14.3\%) of the patients at risk were lying on a more high tech and more expensive alternating mattress during the study period; data for the Avoord nursing homes were $2.3 \%$ (0.4-5.4\%). Therefore, by using this 3-step protocol, also a considerable cost reduction regarding the use of more expensive preventive mattresses was realised.

The conclusions of this study on the new 3-step protocol were clear:

1) Implementation of the 3-step protocol provides a better PU prevention and reduces costs.

2) Standard repositioning for all patients at risk of PUs is not necessary. Starting repositioning, when signs of a category I PU are visible, is early enough.

In literature, no other studies have been performed, looking at a comparable type of protocol or later onset of repositioning.

\section{Methodological Considerations related to the studies of this thesis}

Different methodologies were used in this thesis.

In chapter 2 data about the use of advised PU preventive measures were analysed for Dutch nursing homes. These data were derived from the Dutch National Prevalence Measurement of Care Problems (LPZ). The LPZ is a cross-sectional, multi-centre point prevalence measurement that uses a standardized questionnaire to register amongst other data, the prevalence, as well as the risk of pressure ulcers and also the preventive and therapeutic measurements undertaken in daily PU-care. ${ }^{41}$ The strengths of the LPZ is the fact that data about many residents are available and 
that the participating nursing homes are representative for the Dutch nursing home sector as a whole. Moreover, many nursing homes do participate every year in the LPZ. However, limitations are attached to the cross-sectional design of the LPZ which does not allow making causal conclusions and to the fact that not all nursing homes participate every year.

In chapter 3 and 4 the design of a randomized controlled trial(RCT) was used, which in fact is the right design to study the research questions involved. Normally, a problem of RCTs in which a new intervention is tested against usual care, is that usual care may differ between organizations participating in the study. In the studies of chapter 3 and 4 this was not the case while these studies were tested in one nursing home organization. An important limitation of these 2 studies at that time was, however, the lack of actual and reliable data on PU incidence from other studies to make a correct power analysis before starting both RCTs. Therefore, we had to use data from studies in the 80ths. This aspect probably may be the reason for not finding significant effects in both RCTs, but results may be of clinical relevance.

For the study in chapter 5 on the effect of the 3-step protocol, again the LPZ was used. By applying the methodological approach of the LPZ it is possible to standardize the data collection locally and nationally and this allows benchmarking with other nursing homes in the country. Of course, there are limitations. Data involved prevalence figures and no incidence figures. Furthermore, contrary to the Avoord nursing homes, we lacked insight in the content and intensity of usual care in the other nursing homes. The comparison of the Avoord outcome and process data was done versus the outcome data of all participating nursing homes in the country. Furthermore, the strengths and limitation of the LPZ study (chapter 2) also count for this study. Finally, compared to most other PU intervention studies, the study on the effect of the multilayer skin interface microclimate regulating multi-layer system in chapter 6 is one of the largest multicentre randomised controlled trials ever performed in PU patients in the Netherlands. A strength of this study was the special attention that has been paid to standardising the basic mattress in both the control and intervention group. Residents of both groups received the same new visco-elastic foam mattress. However, because it was necessary to include more than 200 residents at medium/high risk, 21 nursing homes were ultimately needed. Differences in the use of other PU preventive measures in their usual PU care (e.g. differences in skin care, nutritional care, use of repositioning and the way of offloading the heels) may possibly have influenced the results, which can be considered as a limitation. In addition the same problem was present regarding the performance of a good 
power analysis as adequate incidence data for comparable participant groups were not available and we had to use data about the nursing home population as a whole.

\section{Recommendations for daily practice}

The following recommendations for daily practice can be made, based upon the studies of this thesis:

Implementation of most recommendations from current PU guidelines in daily PU care appears to be difficult. Some relevant barriers for implementation, derived from literature, have been described and refering to these barriers it may be advisable to consider first to make the guidelines more simple, understandable and feasible for use in daily practice. Next to this, the implementation should be better supported and controlled, education should be combined with coaching on the job and feasible implementation tools like checklists, apps etc. may be used. This advice is also proposed in other implementation studies. ${ }^{15,16,17,18,19,20,42}$

In addition it should be explored whether a priority choice in the use of the most significant and relevant PU preventive measures would be better than implementing all guideline recommendations in PU prone residents as a whole. This may lead to simpler PU preventive strategies and is confirmed by our experiences with the newly developed 3-step protocol. Concluding from this thesis, this new protocol deserves broader implementation, though some will argue that an additional efficacy study might be necessary following the design of an RCT.

Regarding the use of preventive mattresses, with or without add-ons, the two studies with foam mattresses with or without static air overlays in this thesis, revealed a probably better preventive effect of the additional use of the static air overlay. In the most recent NPUAP-EPUAP-PPIAA guideline for prevention and treatment of PUs of 2014 the basic recommendation for pressure relief with a high specification visco-elastic foam mattress (strengths of evidence A) is subsequently combined with a second recommendation including the possibility of using other types of reactive support surfaces (like static air overlays) (strength of evidence: level B). ${ }^{14}$ This recommendation and its strength fits in the results of the two RCTs, presented in chapter 3 and 4 of this thesis an can in fact easily be applied in daily practice of PU care.

Finally the issue of repositioning. Repositioning is a standard recommendation in all (inter)national PU guidelines. Nevertheless, the results of this thesis show that a nuanced use of respositioning is defendable as well, instead of applying it as a 
standard procedure for every patient at risk of a PU. Looking to the workload of the nursing staff in most nursing homes and the aspect of costs, this is also an interesting finding.

The last study of this thesis was about the theoretical advances of a multi-layer system. Based on this study no advice for use of the full multi-layer system can be given.

Interesting in the last study, was the phenomenon that the new visco-elastic foam mattress, compared to the results of earlier studies with foam mattresses of in general already a higher lifetime, showed a low incidence (4.8\% versus $15-22 \%$ ) of PU development. Based on changes in the memory quality of the foam, manufacturers do advise to replace these mattresses every 5-7 years. Therefore, nursing homes should implement an annual replacement system, to be sure that residents always are lying on an adequate foam mattress. This advice is also clearly stated in the NPUAP-EPUAP-PPPIA guideline on prevention and treatment of PUs 2014 (strengths of evidence C). ${ }^{14}$

\section{Recommendations for further research}

Further research related to PU care may be welcomed on several areas.

First, studies on evaluating relevant guideline implementation strategies remain necessary.

Second, larger and well powered RCTs are necessary related to the use of foam mattresses and add-ons. This also accounts for the definite validation of the newly developed 3-step PU prevention protocol. In addition, this will also contribute to confirmation of our rather innovative standpoint on the more nuanced use of repositioning in PU prone patients. The latter fits also in the view of the Cochrane review on repositioning 2014 in which further research about the best way of using repositioning is stated to be necessary. In this respect, extra attention should be paid as well to the feasibility of applying repositioning in daily practice of PU care.

Third, the recent NPUAP-EPUAP-PPIAA guideline on PU prevention and treatment clearly reveals that more research has to be done on many more recommendations related to PU prevention and treatment. Such research may, amongst other, also provide more insight in the possible benefits of intervening on the microclimate interface for which our study in chapter 6 did not provide conclusive evidence. 
Finally, we would like to stimulate the development of a continuous database about the use and efficacy of PU preventive measures, as derived from daily practice of PU care. Hopefully, this might provide a solution for the fact that it will be tough and very expensive to realise new evidence by a high number of required RCTs as mentioned above. 


\section{REFERENCES}

1. Vanderwee, Clark M, Dealy C, Gunningberg L, Defloor T. Pressure ulcer prevalence in Europe: a pilot study. Journal of Evaluation in Clinical Practice 2007;13:227-235 Incidence.

2. De Souza DM, De Gouveia Santos VL. Incidence of pressure ulcers in the Institutionalized elderly. Journal of Wound, Ostomy and Continence Nursing 2010;37:272-276.

3. Meesterberends E, Halfens RJG, Spreeuwenberg MD, Ambergen TAW, Neyens JCL, Schols JMGA. Do residents in Dutch nursing homes have more pressure ulcers than residents in German nursing homes? A prospective multicentre cohort study JAMDA 2013;14:605-610 doi: 10.1016/j.jamda.2013.03.005.

4. Gorecki C, Brown JM, Nelson EA, Briggs M, Schoonhoven L, Dealey C et al. Impact of pressure ulcers on quality of live in older patients: a systematic review. Journal of the American Geriatric Society. 2009;57(7):1175-83.

5. Dealy C, Posnett J, Walker A. The cost of pressure ulcers in the United Kingdom. Journal of Woundcare. 2012;21(6):261-6.

6. NPUAP/EPUAP. Pressure Ulcer Prevention quick reference guide 2009. http://www.npuap.org/wp-content/ uploads/2012/02/Final_Quick_Prevention_for_web_2010.pdf.

7. V\&VN. Dutch national guidelines for prevention and treatment of pressure ulcers. 2011 http://www. venvn.nl/LinkClick.aspx?fileticket=8TrtENI2ZjE\%3D\&tabid=1852.

8. EPUAP guidelines prevention and treatment 1998. www.epuap.com.

9. Prevention and treatment of pressure ulcers, a Dutch guideline, CBO (Dutch institute for health care improvement), Utrecht 2002.

10. Moore Z, Johansen E, van Etten M. A review of PU risk assessment and prevention in Scandinavia, Iceland and Ireland. Journal of Wound Care vol 22. No 8,432-431. August 2013.

11. Moore Z, Cowman S. Pressure ulcer prevalence and prevention practices in care of the older person in the Republic of Ireland. Journal of Clinical Nursing,21, 362-371, doi:10.1111/j.1365-2702.2011.03749.x

12. Meesterberends E, Halfens RJG, Lohrmann C, Schols JMGA, de Wit R. Evaluation of the dissemination and implementation of pressure ulcer guidelines in Dutch nursing homes. Journal of Evaluation in Clinical Practice 2011;17:705-712. Doi:10.1111/j.1365-2753.2010.01487.x.

13. Demarré L, Vanderwee K, Defloor T, Verhaeghe S, Schoonhoven L, Beeckman D. Pressure ulcer: knowledge and attitude of nurses and nursing assistants in Belgian nursing homes. Journal of Clinical Nursing, 21,1425-1434,doi: 10.1111/j.1365-2702.2011.03878.x.

14. National Pressure Ulcer Advisory Panel, European Pressure Ulcer Advisory Panel and Pan Pacific Pressure Injury Alliance. Prevention and Treatment of Pressure Ulcers: Quick Reference Guide. Emily Haesler (Ed.). Cambridge Media: Perth, Australia; 2014. http://www.npuap.org/wp-content/uploads/2014/08/ Updated-10-16-14-Quick-Reference-Guide-DIGITAL-NPUAP.

15. Oxman AD, Thomson MA, Davis DA, Haynes B. No magic bullets: a systematic review of 102 trials of interventions to improve professional practice. Canadian Medical Association Journal 1995;153:14231431.

16. Davis DA, Taylor-Vaisey A. Translating guidelines into practice: a systematic review of theoretic concepts, practical experience and research evidence in the adoption of clinical practice guidelines. Canadian Medical Association Journal 1997;157:408-416. 
17. Bero LA, Grilli R, Grimshaw JM, Harvey E, Oxman AD, Thomson MA. Closing the gap between research and practice: an overview of systematic reviews of interventions to promote the implementation of research findings. The Cochrane Effective Practice and Organization of Care Review Group. British Medical Journal 1998;317:465-468.

18. National Health Service Centre for Reviews and Dissemination. Getting evidence into practice. Effective Health Care 1999;5:1-16

19. Feder G, Eccles M, Grol R, Griffiths C, Grimshaw J. Using clinical guidelines. British Medical Journal 1999;318:728-730.

20. Grimshaw JM, Shirran L, Thomas R, Mowatt G, Fraser C, Bero L, Grilli R, Harvey E, Oxman A, O’Brien MA. Changing provider behaviour: an overview of systematic reviews of interventions. Medical Care 2001;39:2-45.

21. Geboers H, Grol R, van den Bosch W, van den Hoogen H, Mokkink H, van Montfort P, Oltheten H. A model for continuous quality improvement in small scale practices. Qual Health Care. 1999 Mar; 8(1): 43-48.

22. Takala, J., Varmavuo, S. \& Soppi, E., 1996. Prevention of pressure sores in acute respiratory failure: a randomised controlled trial. Clinical Intensive Care., Issue 7, p. 228-235.

23. Sanada H, Sugama J, Matsui Y, Konya C, Kitagawa A, Okuwa M et al. A randomised controlled trial to evaluate a new double-layer air-cell overlay for elderly patients requiring head elevation. Journal of Tissue Viability. Volume 13, Issue 3, July 2003, Pages 112-121.

24 Serraes B, Beeckman D. Static air support surfaces to prevent pressure injuries. J Wound Ostomy Continence Nurs. 2016;43(4):375-378.

25. Vermette S, Reeves I, Lemaire J. Cost Effectiveness of an Air-inflated Static Overlay for Pressure Ulcer Prevention: A Randomized, Controlled Trial. WOUNDS 2012;24(8):207-14.

26. McInnes E, Jammali-Blasi A, Bell-Syer SEM, Dumville JC, Middleton V, Cullum N. Support surfaces for pressure ulcer prevention. Cochrane Database of Systematic Reviews 2015, Issue 9.Art.no.:CD001735. DOI:10.1002/14651858.CD001735.pub5.

27. Clark M, Romanelli M, Reger SI, Ranganathan VK, Black J, Dealey. Microclimate in context. International review. Pressure, shear, friction and microclimate in context. A consensus document. London: Wounds international, 2010

28. Rapp MP, Bergstrom N, Padhye NS. Contribution of skin temperature regularity to the risk of developing pressure ulcers in nursing facility residents. Adv Skin Wound Care 2009;22(11):506-13.

29. Sae-Sia W, Wipke-Tevis DD, Williams DA. Elevated sacral skin temperature (Ts): a risk factor for pressure ulcer development in hospitalized neurologically impaired Thai residents. Appl Nurs Res 2005;18:29-35.

30. Orsted HL, Ohura T, Harding K. International review. Pressure ulcer prevention: Pressure, shear, friction and microclimate in context. A consensus document. Londen Wounds International, 2010.

31. Mayrovitz HN, Sims N. Biophysical effects of water and synthetic urine on skin. Adv Skin Wound Care 2001; 14(6): 302-8.

32. Egawa M, Oguri M, Kuwahara T, Takahashi M. Effect of exposure of human skin to a dry environment. Skin Res Technol 2002; 8(4): 212-18.

33. Institut für Textil- und Verfarenstechnik Denkendorf. Unsere auftrags-NR.:E-0230-TT-15, 07-10-2015. Available at: www.sensetextile.com 
34. Institut für Textil- und Verfarenstechnik Denkendorf. Untersuchungsbericht NR.E-0076-TT-16, 11-042016. Available at: www.sensetextile.com

35. Vanderwee K, Grypdonck MH, Defloor T. Effectiveness of an alternating pressure air mattress for the prevention of pressure ulcers. Age Ageing. 2005 May;34(3):261-7. Epub 2005 Mar 11.

36. McInnes E, Jammali-Blasi A, Bell-Syer SEM, Dumville JC, Middleton V, Cullum N. Support surfaces for pressure ulcer prevention. Cochrane Database of Systematic Reviews 2015, Issue 9. Art. No.: CD001735. DOI: 10.1002/14651858.CD001735.pub5.

37. Defloor T, De Bacquer D, Grypdonck MH. The effect of various combinations of turning and pressure reducing devices on the incidence of pressure ulcers. Int J Nurs Stud. 2005 Jan;42(1):37-46.

38. Källman U, Bergstrand S, Ek AC, Engström M, Lindberg LG, Lindgren M. Different lying positions and their effects on tissue blood flow and skin temperature in older adult patients. J. Adv. Nurs. 2013 69, 133-144.

39. Gillespie BM, Chaboyer WP, McInnes E, Kent B, Whitty JA, Thalib L. Repositioning for pressure ulcer prevention in adults. Cochrane database of systematic reviews 2014, issue 4. No.:CD009958. DOI: 10.1002/14651858.CD009958.pub2.

40. Moore Z, Cowman S. Pressure ulcer prevalence and prevention practices in care of the older person in the republic of Ireland. J Clin Nurs. 2012 Feb;21(3-4):362-71. Doi:10.1111/j.1365-2702.2011.03749.x. Epub $2011 \mathrm{Jul} 7$.

41. Halfens RJG, Meijers JMM, Du Moulin MFMT, van Nie NC, Neyens JCL, Schols JMGA. National Prevalence Measurement of Care Problems. Caphri school for public health and primary care Maastricht, Netherlands 2010.

42. Breimaier HE, Halfens RJG, Wilborn D, Meesterberends E, Haase Nielsen G, Lohrmann C. Implementation Interventions Used in Nursing Homes and Hospitals: A Descriptive, Comparative Study between Austria, Germany, and The Netherlands. ISRN Nursing Volume 2013 (2013), Article ID 706054, 12 pages. http:/| dx.doi.org/10.1155/2013/706054.), Article ID 706054, 12 pages. http://dx.doi.org/10.1155/2013/706054. 
O

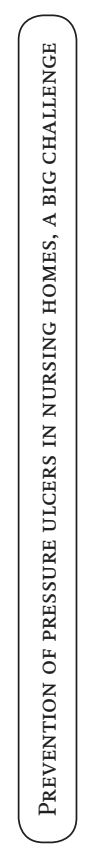


CHAPTER 8

\section{VALORISATION}


O

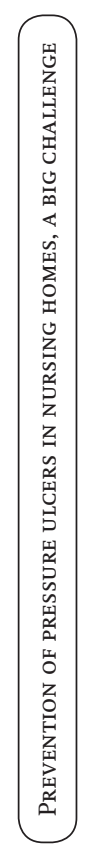




\section{Valorisation}

In this chapter, the findings of this thesis on the use and efficacy of pressure ulcer preventive measures in nursing home residents and there societal value are addressed. Consecutively, the analysis of used pressure ulcer preventive measures over the years, the effect of static air mattresses on the development of pressure ulcers, the effect of a newly developed simple 3-step PU preventive protocol and the PU preventive effect of a newly developed pressure/shear relieving and microclimate regulating system are described. This chapter ends with a short description of the dissemination of the results of this thesis.

Pressure ulcers (PUs) are often painful, sometimes smelling and causing great discomfort in nursing home residents. In addition, they may lead to loss of social participation and quality of life. Moreover, the treatment of PUs is associated with high costs. Therefore, optimal PU prevention is of utmost importance.

Currently, extensive and voluminous (inter)national guidelines are available to target adequate pressure ulcer prevention in different health care sectors, including the nursing home sector. However, the problem of these voluminous guidelines, containing a bulk of recommendations on skin care, PU risk assessment, pressure relieving measures and nutritional care, is that most of the recommendations lack sufficient evidence. Unknown, for instance, is the contribution of each specific PU preventive measure on the incidence of PUs. The same counts for what the optimal combination of PU preventive measures is. Moreover, in daily practice, these guidelines are often insufficiently implemented and difficult to understand and follow for health care professionals. The results of this thesis confirmed the incomplete use of PU preventive measures in nursing homes over the years, probably because of an insufficient implementation and lack of awareness.

An adequate strategy to improve daily PU care might be to provide professionals with a more feasible, easy to understand and user friendly PU prevention protocol. In this thesis an easy to follow 3 step PU prevention protocol is introduced, that shows a clearly and sustainable PU reducing effect. 
This 3 step protocol involves:

Step 1: The resident receives a standard visco-elastic mattress, without repositioning;

Step 2: The resident who develops signs of a possible category $1 \mathrm{PU}$ (non-blanchable redness) receives a static air overlay (on top of the visco-elastic foam mattress), again without repositioning;

Step 3: The resident who still develops a PU is repositioned every 3 hours during the day and every 4 hours during the night.

Alongside the positive outcome of using this new 3 step PU prevention protocol, the results of the thesis also confirm the usefulness of additional static air mattresses on top of a regular mattress and the positive effect of using a more tailored approach 120 regarding repositioning because these effects also may involve a positive effect on the well-being of the residents and a reduction of the costs. Therefore, the new 3 step protocol deserves broader dissemination and implementation. Subsequently a broader and more in-depth scientific evaluation of the added value of this protocol for the nursing home sector should be done. In the future, it can also be assessed, whether there is room for relevant additional steps, taking into account that this requires a thorough evidence base and that preservation of the feasibility and resident friendliness remains necessary. One might think about a step related to preserving tissue viability by adequate skin care and /or nutritional care, or by incorporating the use of even better PU preventive mattresses/materials. Referring to the last issue, the study in this thesis about the PU preventive effect of a newly developed pressure/shear relieving and microclimate regulating system offered no reason for incorporation.

Two studies in this thesis also show the benefits of the Dutch National Prevalence Measurement of Care problems (LPZ) as executed by Maastricht University. Participating in this measurement offers considerable advantages for the participating health care organisations, including nursing homes, because it offers them reliable benchmark information, it may increase awareness among professionals and it provides relevant data for research.

The quality of care for nursing home residents may profit from research done in this target group. Until recently, nursing home residents often were excluded from clinical studies because of their heterogeneous profile, characterized by multi-morbidity, considerable handicaps and polypharmacy. This is also reflected by many of the current guidelines, including the guidelines on PU care, which mostly incorporate recommendations derived from studies in other target groups 
that cannot be extrapolated directly to the target group of nursing home residents. Therefore it is important that the studies of this thesis clearly show that research in nursing home residents is possible and this should be taken into account when developing future research programs in frail and disabled older persons. This will also enable the development of specific guidelines for the nursing home population.

In addition, future PU research should focus more on evaluating relevant guideline implementation strategies. Next to this, many of the current PU preventive measures need a more evidence base in both overall clinical practice and more specifically in the nursing home sector. This not only counts for specific PU preventive measures like repositioning and the use of a variety of mattresses and microclimate influencing materials but also for the target group related search for the adequate combination of PU preventive measures. The problem however is that this will require a large number of adequately conducted RCTs, which is very expensive and challenging... An interesting option might be the development of a continuous database about the use and efficacy of PU preventive measures in daily practice.

\section{Dissemination}

Next to their scientific value, the results of this thesis can be used to additionally raise the awareness for the problem of pressure ulcers in nursing homes. The outcomes of the studies were and will be presented on different congresses and symposia and will be used in the development of educational programs for the nursing staff and other nursing home professionals, including nursing home physicians, and paramedical staff. 
O

122

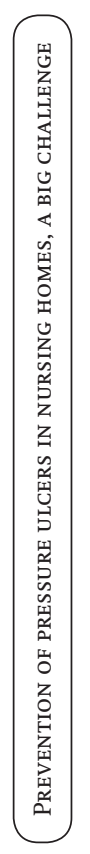


O

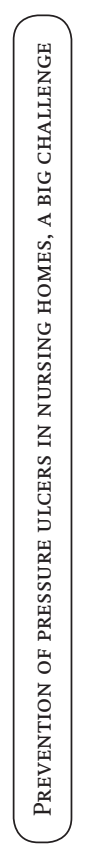




\section{Summary}

This thesis aims to improve the process of pressure ulcer (PU) prevention in Dutch nursing homes.

Pressure ulcers (PUs) are a very distressing problem for nursing home residents. They often cause a lot of pain and discomfort and are associated with labour intensive professional care, resulting in high costs. Therefore, prevention of PUs in nursing homes is of utmost importance

Nowadays, (inter)national guidelines on PU prevention are available which advise a variety of preventive measures, including amongst others: a standard PU risk analysis by using more or less validated risk scores, paying attention to skin care and nutritional care and taking care of adequate pressure relief in bed and (wheel) chair by using pressure relieving mattresses/cushions and applying repositioning. Though it is known that being aware of the PU risk in frail residents and paying extra attention to PU prevention may lead to a lower PU prevalence, unfortunately, for many of the recommended preventive measures there still is less scientific evidence. Unknown is also the contribution of each specific PU preventive measure on PU incidence and which is the optimal combination of PU preventive measures.

Therefore, still a lot of scientific questions remain to be solved to achieve a more solid evidence base for the prevention of pressure ulcers.

In this thesis, the following research questions have been addressed:

1. To what extent did Dutch nursing homes apply PU preventive measures, as advised in (inter)national PU guidelines, during the period 2005-2014?

2. What is the clinical efficacy of combining a standard $15 \mathrm{~cm}$ cold foam mattress with a static air overlay mattress versus a cold foam mattress alone in preventing pressure ulcers in nursing home residents?

3. What is the clinical efficacy of a combination of a standard $15 \mathrm{~cm}$ visco-elastic foam mattress with a static air overlay mattress versus a standard $15 \mathrm{~cm}$ viscoelastic foam mattress alone in preventing pressure ulcers in nursing home residents?

4. What is the effect of implementing a 3 step PU prevention protocol on PU prevalence in the Avoord nursing homes (Etten-Leur, The Netherlands) over the period 2005-2011?

5. What is the effect of a newly developed multilayer skin interface microclimate regulating system, used in conjunction with a regular high quality visco-elastic foam mattress on PU incidence in nursing home residents? 
In chapter one, the introduction of the thesis, general background information about pressure ulcers and the factors which may contribute to their development is provided. In addition, the issue of PU prevention in the nursing home setting is introduced by giving some basic data on the Dutch nursing home sector.

Finally, the objectives and outline of the thesis are described.

In chapter 2 the results of an analysis of the use of guideline recommended PU preventive measures over a 10 year period in 3 groups of Dutch nursing home residents are presented. The 3 groups involved: (1) residents with a Braden score of 17-19; (2) residents with a Braden score less than 17 and (3) residents with a PU. The data were derived from the annual independent Dutch National Prevalence Measurement of Care Problems (LPZ) as executed by Maastricht University. This study showed that none of the advised measures were fully used during this period. Despite that, the overall use of preventive measures was higher when the risk of PU development was higher or when PUs were already present. Moreover, the data showed an increase of the use of skin care, use of static air overlays and preventive air cushions in all three groups, and also an increase in the use of offloading of the heels in group two and three. A decrease over the years was found in the application of repositioning and the use of alternating mattressess in group two, and in the use of foam cushions in group one and two.

One of the advised measures in the (inter)national guidelines is the standard combination of pressure relieving systems in bed/(wheel)chair and repositioning. However, contrary to what may be expected, the evidence for repositioning is still poor, even as the compliance of the nursing staff in the execution of it in daily PU care. In addition repositioning during the night may cause unnecessary patient discomfort and lead to undesired behavioural problems, particularly in demented residents.Therefore, in the RCTs of chapter 3 and 4 repositioning was not incorporated as a standard measure in combination with the use of pressure reducing (static air) mattresses and only done when signs of a PU occurred.

Chapter 3 presents the results of a randomized controlled trial, looking at the PU preventive effect of a cold foam mattress versus the same mattress with a static air overlay on top of it. The study was executed in nursing home De Naaldhorst, Naaldwijk, The Netherlands. 83 residents with a medium or high risk of PU development (Norton score less than 12 points) were included and followed for a period of 6 months. After informed consent and randomisation 41 residents received the additional static air overlay. None of the residents received standard repositioning 
at the start of the study. Seven patients (17.1\%) on the cold foam mattress alone and two (4.8\%) lying on the static air mattress developed a pressure ulcer category 2 or higher. There was no difference in pressure ulcer incidence between residents with a high risk (Norton 5-8) and medium risk (Norton 9-12) of PUs. In this study, static air overlay mattresses provided a better PU prevention than cold foam mattresses alone $(4.8 \%$ versus $17.1 \%)$, which however was not statistically significant $(\mathrm{p}=0.88)$. Yet, the difference in PU development is of clinical importance. The Norton scores of the residents in both groups did not change during the 6 month trial period. The use of repositioning only when there was a sign of a pressure ulcer seems to be acceptable when a static air overlay is in position. However, the incidence of $17.1 \%$ PU development in the foam group may stress the need of repositioning when using only this type of mattress.

In chapter 4 the results of a prospective, cross-over randomized controlled trial on the preventive effect of a visco-elastic foam mattress versus the same mattress with a static air overlay on top of it are presented. 41 residents with a Braden score less than 20 were randomized for a study period of 1 year $(6$ months on a visco-elastic foam mattress and 6 months on the same mattress with a static air overlay on top). Repositioning was not part of standard PU care. The results showed that 8 patients (22.2\%) developed a category 2 or higher pressure ulcer on a visco-elastic foam mattress (control group) and 2 (5.2\%) on a static air mattress (intervention group) $(\mathrm{p}=0.087)$. This was not statistically significant, but may be of clinical importance. The use of repositioning only when there were signs of a pressure ulcer appeared to be acceptable in cases where a static air overlay is used. The $22.2 \%$ incidence of pressure ulcers in the foam group, however, again may stress the need of repositioning when using this type of basic mattress alone.

Chapter 5 describes the effect of a simple and feasible 3-step PU preventive protocol on the PU prevalence in the Avoord nursing homes (Etten-Leur, The Netherlands) over the period 2005-2011.

In addition to proper PU prevention, the reason for developing this new protocol, partly based on the results of the studies in chapter 3 and 4, was to tailor the application of repositioning and thereby the compliance of the nursing staff in using repositioning in a proper way.

The new PU protocol involves 3 main steps: 1) All residents receive a standard visco-elastic mattress, without repositioning; 2) residents who develop signs of a possible category $1 \mathrm{PU}$ (non-blanchable redness) receive a static air overlay (on top 
of the visco-elastic foam mattress), again without repositioning; 3) residents who still develop a PU are repositioned every 3 hours during the day and every 4 hours during the night. If this 3-step protocol is insufficient because a resident develops a more severe PU (i.e., category 4) the resident's mattress will be changed for a low-air loss system.

Data of residents of the Avoord nursing homes in Etten-Leur/Zundert (Netherlands) were analysed and compared with national data between 2002 and 2011(data used from the yearly measurement of the Dutch national prevalence measurement of care problems $[\mathrm{LPZ}\})$.

The study showed a significant reduction of the nosocomial prevalence of category 2-4 PUs over the years after the introduction of the new protocol in the Avoord nursing homes. The PU prevalence reduced from $8.7 \%$ to $0.5 \%$ during the first year and remained stable at about $2 \%$ throughout the rest of the study period. The overall PU prevalence at national level also decreased during this period, but not as much, and was still significantly higher in 2011. In the Avoord nursing homes, the use of more expensive alternated systems decreased to almost $1 \%$, while the use of static air mattresses showed an almost linear rise from the start of the implementation of the protocol. Contrary, the trend for both types of mattresses remained stable on a national level. Introducing the static air mattress instead of the more expensive alternating mattress helped to reduce the mean daily costs of PU preventive mattresses in the Avoord nursing homes by more than $70 \%$ compared to national figures. The workload of the nursing staff decreased as well due to the reduction of the application of repositioning.

Chapter 6 focuses on a rather new development in PU care. Recently more attention is paid to the influence of the microclimate on PU development. With microclimate is meant the temperature and humidity of the interface between skin and mattress. Laboratory tests in the last decade have revealed that disturbances of the microclimate may contribute to the development of pressure ulcers. Therefore, in this chapter, the results of a randomized controlled trial on the effect of a newly developed multilayer skin interface microclimate regulating system, used in conjunction with a regular high quality visco-elastic foam mattress on the incidence of PUs in nursing home residents are presented. A multicentre, prospective, controlled randomized clinical trial was executed in 21 nursing homes. Residents with a Braden score less than 16, a life expectancy of more than 3 months and no PU during the last 3 months were asked to participate and included after informed consent. The control group received a new high quality visco-elastic foam mattress covered with a cotton 
sheet (standard care). The intervention group received the same new high quality visco-elastic foam mattress, in combination with the newly developed multilayer system. 206 residents participated in the 12-week study. In the control group ( $\mathrm{n}=103$ ) $5 \%$ of the residents developed a category 2,3 or $4 \mathrm{PU}$ and in the intervention group ( $\mathrm{n}=103$ ) $9 \%$ (a statistically not significant difference). The study revealed that the extra applied new multilayer system had no added value over the use of the viscoelastic foam mattress alone.

The final chapter (chapter 7) concerns the general discussion of the thesis and provides an overview of the main findings presented in the other chapters. Theoretical considerations, methodological considerations and also recommendations for both daily practice and future research are presented. 
O

130

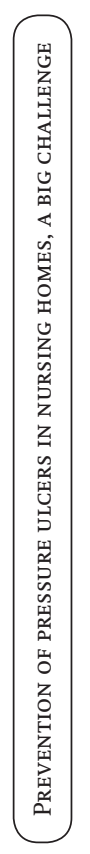


O

$$
132
$$

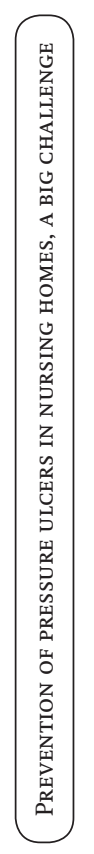




\section{Samenvatting}

Dit proefschrift heeft als doel een bijdrage te leveren aan de preventie van doorligwonden bij bewoners van Nederlandse verpleeghuizen. Doorligwonden (decubitus) vormen een vervelend probleem voor verpleeghuisbewoners. Ze veroorzaken vaak pijn en ongemak, en hebben intensieve professionele zorg nodig, wat resulteert in hoge kosten. Een goede preventie van decubituswonden in verpleeghuizen is daarom van zeer groot belang. (Inter)nationale preventieve richtlijnen voor decubitus raden diverse preventieve maatregelen aan, zoals een gestandaardiseerde risicoanalyse met een gevalideerde risicoscorelijst, aandacht voor huidzorg en voedingstoestand, adequate drukvermindering in bed en (rol)stoel door drukverlagende matrassen/kussens en het toepassen van wisselhouding. Hoewel het bekend is dat aandacht voor het risico op decubitus bij kwetsbare bewoners en het toepassen van preventieve maatregelen leidt tot vermindering van decubitus, is er helaas voor veel geadviseerde maatregelen nog weinig wetenschappelijk bewijs. Behalve dat het effect van de meeste maatregelen onbekend is, is ook onduidelijk welk effect een combinatie van maatregelen heeft. Voordat een evidence based preventiebeleid van decubitus mogelijk is, zullen nog veel vragen wetenschappelijk onderzocht moeten worden.

In dit proefschrift worden de volgende vragen onderzocht:

1. In welke mate werden in Nederlandse verpleeghuizen preventieve decubitus maatregelen, zoals geadviseerd in (inter)nationale preventieve richtlijnen, toegepast, in de periode 2005-2014?

2. Wat is de klinische waarde van de combinatie van een standaard $15 \mathrm{~cm}$ dikke koudfoam matras met een statische luchtmatras ten opzichte van een koudfoam matras ter preventie van decubitus bij verpleeghuisbewoners?

3. Wat is de klinische waarde van de combinatie van een standaard $15 \mathrm{~cm}$ dikke viscoelastische foammatras met een statische luchtmatras ten opzichte van een viscoelastische foammatras ter preventie van decubitus bij verpleeghuisbewoners?

4. Wat zijn de gevolgen van de implementatie van het 3-step decubitus preventie protocol in de verpleeghuislocaties van Avoord Zorg en Wonen (Etten-Leur, Nederland) in de periode 2005-2011?

5. Wat is het preventieve effect op het ontwikkelen van decubitus bij verpleeghuisbewoners van een nieuw ontwikkeld meerlagen, op huidniveau, microklimaat regulerend systeem in combinatie met een nieuwe hoog kwalitatieve visco-elastische foammatras?

In hoofdstuk 1, de inleiding op dit proefschrift, staat algemene achtergrondinformatie over decubitus en de factoren die van invloed zijn op het ontstaan van decubitus. 
Tevens wordt de preventie van decubitus in verpleeghuizen besproken. Tenslotte worden de doelen en de opzet van dit proefschrift besproken.

In hoofdstuk 2 worden de resultaten van een onderzoek naar de inzet van door richtlijnen aanbevolen preventieve decubitusmaatregelen over een periode van 10 jaar bij 3 groepen Nederlandse verpleeghuisbewoners besproken, te weten: (1) bewoners met een score van 17-19 op de Bradenschaal, (2) bewoners met een score lager dan 17 en (3) bewoners met een decubituswond. De gebruikte data zijn afkomstig uit de jaarlijkse meting van de Nederlandse Landelijke Prevalentiemeting Zorgproblemen (LPZ). Uit het onderzoek bleek dat de in de (inter)nationale richtlijnen geadviseerde maatregelen, zoals aandacht voor huid en voedingsstaat, drukreductie etc. niet voldoende werden ingezet. Wel werden ze vaker ingezet 134 naarmate het risico op decubitus toenam (lagere Braden score) en bij de groep met decubituswonden. Tijdens de gemeten periode nam de aandacht voor de zorg van de huid, de voedingsstatus, de inzet van statische luchtmatrassen/kussens in alle 3 de groepen toe en werd drukontlasting van de hielen in groep 2 en 3 vaker toegepast. Het gebruik van wisselhouding nam in die10 jaar echter af en alternerende luchtmatrassen (in groep 2) en foamkussens (in groep 1 en 2) werden minder vaak gebruikt.

Een van de geadviseerde preventieve maatregelen is de standaard combinatie van drukverdelende systemen in bed/(rol)stoel met wisselhouding. In tegenstelling tot wat men zou verwachten, is de wetenschappelijke onderbouwing aangaande de inzet van wisselhouding echter matig, terwijl de toepassing van wisselhouding door de verpleegkundige staf tijdens de dagelijkse basiszorg te wensen overlaat. Daarnaast kan de inzet van wisselhouding 's nachts leiden tot ongewenst ongemak, en mogelijk tot ongewenste gedragsproblemen, met name bij demente bewoners. Daarom is besloten om in de RCT's, zoals besproken in hoofdstuk 3 en 4, geen standaard wisselhouding toe te passen. Alleen als er ondanks inzet van een statische luchtmatras tekenen van decubitus waren, werd wisselhouding gestart.

In hoofdstuk 3 worden de resultaten beschreven van een gerandomiseerd gecontroleerd onderzoek naar het preventieve effect van een statische luchtmatras op een standaard koudfoam matras ten opzichte van alleen een standaard koudfoam matras op het ontstaan van decubitus. De studie vond plaats in verpleeghuis De Naaldhorst in Naaldwijk, Nederland. 83 bewoners met een gemiddeld of hoog risico op het ontwikkelen van decubitus (Norton $<12$ punten) werden geïncludeerd en gedurende 6 maanden gevolgd. Na het verkrijgen van informed consent en de randomisatie kregen 41 bewoners in de interventiegroep een statische luchtmatras 
op de standaard koudfoam matras. Bij geen van de bewoners werd aan het begin van de studie wisselhouding toegepast. Zeven bewoners $(17,1 \%)$ met alleen een koudfoam matras en twee bewoners $(4,8 \%)$ in de interventiegroep ontwikkelden een categorie 2 of ernstigere decubituswond. Het verschil tussen controle- en interventiegroep was niet statistisch significant $(\mathrm{p}=0,088)$, maar is wel klinisch relevant. Met betrekking tot de incidentie is er tussen de hoge risicogroep (Norton 5-8) en de medium risicogroep (Norton 9-12) geen verschil gevonden.

Gebaseerd op studie 1 werd wisselhouding pas ingezet bij tekenen van decubitus. Bij inzet van een statische luchtmatras lijkt deze beslissing verantwoord, echter gezien $17,1 \%$ van de bewoners met alleen een koudfoam matras decubituswonden ontwikkelde lijkt inzet van wisselhouding in een eerdere fase noodzakelijk.

In hoofdstuk 4 worden de resultaten beschreven van een prospectief, crossover gerandomiseerd gecontroleerd onderzoek naar het effect van een statische luchtmatras op een standaard visco-elastische matras ten opzicht van alleen een standaard visco-elastische matras op het ontstaan van decubitus. 41 bewoners met een Braden score lager dan 20 werden gerandomiseerd. De periode van deelname was 1 jaar ( 6 maanden preventie met visco-elastische matras en 6 maanden met dezelfde matras met een statische luchtmatras erop). Conform de in hoofdstuk 3 besproken RCT werd wisselhouding tijdens de studieperiode alleen toegepast bij tekenen van decubitus. 8 bewoners $(22,2 \%)$ in de groep met alleen de viscoelastische foammatras (controlegroep) ontwikkelden een categorie 2 of ernstigere decubituswond. In de interventiegroep (visco-elastische matras plus een statische lucht matras) ontwikkelden slechts 2 bewoners $(5,2 \%)$ een decubituswond. Dit verschil was niet statistisch significant $(\mathrm{p}=0,087)$, maar is wel klinisch relevant. Uit deze en de vorige studie wordt duidelijk dat het gebruik van een extra statische luchtmatras zinvol is. Het toepassen van wisselhouding op het moment van ontstaan van een decubituswond lijkt in de interventiegroep verantwoord, maar is dat, gezien het hoge percentage decubituswonden in de controlegroep, niet.

Hoofdstuk 5 beschrijft het effect van een simpel en uitvoerbaar 3-staps decubitus preventieprotocol in de verpleeghuislocaties van Avoord Zorg en Wonen (Etten-Leur, Nederland) gedurende de periode 2005-2011. Dit protocol is ontwikkeld op basis van de resultaten uit hoofdstuk 3 en 4 . Uitgangspunt was dat wisselhouding op maat toegepast moet worden, zodat de compliance om wisselhouding door de verzorging/ verpleging toe te passen, geoptimaliseerd wordt. Het nieuwe protocol omvat de volgende 3 stappen: 1) alle bewoners liggen standaard op een visco-elastische foammatras, maar krijgen geen wisselhouding, 2) bij tekenen van een mogelijk 
ontstaan van decubitus wordt een statische luchtmatras op de visco-elastische foammatras gelegd, maar wordt nog geen wisselhouding toegepast en 3) bij ontstaan van decubitus, ondanks goede toepassing van voorgaande stappen, wordt gestart met wisselhouding, elke 3 uur overdag en elke 4 uur 's nachts. Als toepassing van het 3-staps protocol nog niet voldoende blijkt te zijn omdat er een categorie 4 decubituswond ontstaat, worden de matrassen vervangen door een low-air-loss matras. De resultaten van bewoners van de Avoord locaties werden vervolgens vergeleken met de landelijke resultaten tussen 2002 en 2011 (data verkregen uit de Landelijke Prevalentiemeting Zorgproblemen [LPZ]). De resultaten lieten een significante vermindering van de nosocomiale prevalentie van decubituswonden categorie 2-4 zien gedurende de jaren na implementatie van het 3-staps protocol in de verpleeghuislocaties van Avoord. De decubitusprevalentie daalde in het eerste jaar van $8,7 \%$ naar $0,5 \%$ en bleef stabiel rond $2 \%$ gedurende de resterende studieperiode. De landelijke prevalentiecijfers aangaande decubitus daalden ook, maar veel geleidelijker en waren in 2011 nog steeds significant hoger. Op de locaties van Avoord daalde de inzet van duurdere alternerende luchtmatrassen, terwijl de inzet van statische luchtmatrassen na de implementatie vrijwel lineair steeg. Landelijk bleef de inzet van alternerende en statische luchtmatrassen gelijk. Het introduceren van statische luchtmatrassen in plaats van de duurdere alternerende matrassen op de locaties van Avoord had een kostenreductie van bijna $70 \%$ tot gevolg in vergelijking met de kosten van het landelijk beleid. De workload van de verzorging/verpleging daalde eveneens door het verminderen van het toepassen van wisselhouding.

Hoofdstuk 6 gaat over een vrij nieuwe ontwikkeling in de decubituszorg. Recent wordt er meer aandacht besteed aan de invloed van het microklimaat op het ontstaan van decubitus. Met het microklimaat wordt de temperatuur en de vochtigheidsgraad van de huid op het niveau van de onderliggende matras bedoeld. Laboratoriumproeven, verricht in de laatste 10 jaar, hebben aangetoond dat veranderingen van het microklimaat kunnen bijdragen aan het ontstaan van decubitus. Daarom is een gerandomiseerd gecontroleerd onderzoek (RCT) uitgevoerd naar de preventieve effecten van een nieuw ontwikkeld, multilagen, microklimaat regulerend systeem in combinatie met een hoogkwalitatieve visco-elastische foammatras, toegepast bij verpleeghuisbewoners. Deze RCT werd uitgevoerd in 21 verpleeghuislocaties. Bewoners met een Braden score $<16$, een levensverwachting van $>3$ maanden en geen decubituswonden in de laatste 3 maanden, werden verzocht deel te nemen en na informed consent vervolgens geïncludeerd. De controlegroep kreeg een nieuwe visco-elastische foammatras, bedekt met een katoenen laken (standaard zorg). De interventiegroep kreeg eenzelfde type nieuwe visco-elastische 
foammatras, maar in combinatie met het nieuw ontwikkeld multilagensysteem. 206 bewoners participeerden in deze 12 weken durende studie. In de controlegroep $(\mathrm{n}=103)$ ontwikkelde $5 \%$ van de bewoners een categorie 2 of 3 decubituswond en in de interventiegroep ( $\mathrm{n}=103$ ) 9\% (statistisch niet significant). Het gebruik van het meerlagen systeem heeft dus geen meerwaarde.

Het laatste hoofdstuk (hoofdstuk 7) bevat de algemene discussie van dit proefschrift en geeft een overzicht van de belangrijkste bevindingen van de vorige hoofdstukken. In dit hoofdstuk zijn zowel theoretische en methodologische overwegingen, alsmede aanbevelingen voor de dagelijkse praktijk en verder onderzoek opgenomen. 
O

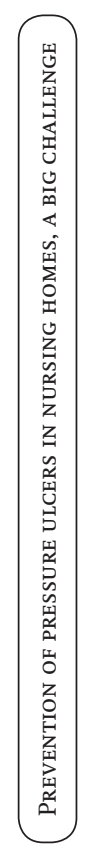




\section{DANKWOORD}


O

140

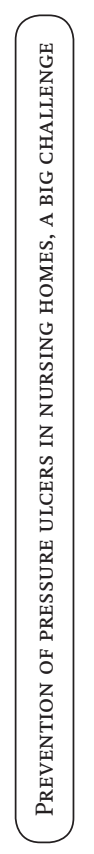




\section{Dankwoord}

\section{Het was een wens en de wens is nu vervuld}

Dit proefschrift was nooit tot stand gekomen zonder de ondersteuning van velen.

Allereerst gaat mijn dank uit naar de bewoners van de vele verpleeghuizen in Nederland, die actief geparticipeerd hebben in de drie interventiestudies en de bewoners die deelnamen aan het Landelijk Prevalentiemeting Zorgproblemen.

Tevens gaat mijn dank uit naar alle medewerkers van de betrokken zorgorganisaties (Elisabeth Breda, Groenhuysen, Volkaert, Surplus, Vivent, Vughterstede, Savant, de Wever, Meander, Thebe, de Zorgboog, Zorgpartners Midden Holland, Het Parkhuis, Swinhove, Riethorst-Stromenland, de Naaldhorst en Avoord Zorg en Wonen) voor jullie medewerking, behulpzaamheid en gastvrijheid. Zonder jullie betrokkenheid, enthousiasme en stimulans om door te gaan, zou ik de eindstreep niet gehaald hebben. Ook Jolanda Kuijer, Patricia Broos en Barry Willemstein veel dank. Een grote RCT in 21 verpleeghuizen had zonder jullie inzet en controlerend vermogen nooit een betrouwbaar resultaat opgeleverd.

In 1998 werd ik geconfronteerd in verpleeghuis De Naaldhorst met decubituswonden, die veel effect hadden op de kwaliteit van leven van de getroffen bewoners. Toen ik mijn onmacht een aantal jaren later besprak met Jos Schols adviseerde hij mij om meer onderzoek te gaan doen naar effectieve preventieve maatregelen. Ik had toen al 2 studies gedaan in de hoop betere maatregelen te vinden ter voorkoming van decubitus, maar nog niet de resultaten gedeeld via een publicatie. Aangezien Jos toen nog geen hoogleraar was, ben ik in gesprek gegaan met Steven Hovius, en deze was bereid om mij te ondersteunen in een promotietraject, aanvankelijk vanuit de Erasmus Universiteit, later als mede hoofdpromotor vanuit Maastricht.

Een woord van dank gaat uit naar mijn begeleiders gedurende het hele promotietraject voor de begeleiding, de vele opbouwende kritiek, het mij uit de put halen op momenten dat ik het allemaal niet meer zo zag zitten en het vertrouwen op een goed einde.

Beste Jos, jij was al die jaren, zowel voor als vanaf het moment dat je promotor werd, een gedreven, zeer kritische begeleider. Steeds weer bouwde je samen met mij aan een concept artikel, zodat ik geen flater zou slaan bij de andere 2 begeleiders. Het was haast ondoenlijk om enige rustpauze in te bouwen als ik weer een concept artikel naar je stuurde, want ik had de computer vaak slechts enkele momenten 
afgesloten of er was al een reactie van jou. Steeds weer gaf me steun om door te gaan, en ja hier is dan toch het resultaat. Je enthousiaste verhalen naar collega's over het vorderen van de promotie, waren een enorme bron van inspiratie om door te gaan. Op momenten dat ik het niet meer zag zitten om verder te gaan met schrijven, pepte jij me weer op door opbeurende woorden dat we het eind al "bijna" in zicht hadden.

Beste Steven, mijn woord van dank gaat ook uit naar jou, vanwege de kans die jij me gaf om aan het promotietraject te beginnen. Je liet het initiatief duidelijk bij mij, gaf veel commentaar op mijn $1^{\text {ste }}$ artikel (je waarschuwde me al bij het eerste gesprek over een eventuele promotie al over veel 'Rood' in je commentaren), dacht mee toen ik opperde om aan de Universiteit Maastricht te willen promoveren en bleef me steunen in het inmiddels doorlopen traject.

Beste Ruud, jij was mijn rust in het proces. Wanneer er een heftige periode was, relativeerde je alles en hielp je constructief tot een goed resultaat. Jouw statistische bewerkingen hebben het mogelijk gemaakt om mijn artikelen te kunnen submitten.

Susan, wat ben ik blij met jouw hulp bij het tot stand komen van het manuscript. Jouw kritische en taalkundige blik hebben mij behoed voor fouten in het thesisboekje. Ook regelde jij de benodigde documenten voor mij en had je een luisteren oor.

Graag wil ik de leden van de beoordelingscommissie, Prof. dr. R.A. de Bie, Prof. dr. Ir. C.W.J. Oomens, Prof. dr. R.R.J.W. van der Hulst, Dr. H.E.W. de Laat en Dr. A.A.L.M. Rondas hartelijk danken voor het lezen en beoordelen van dit proefschrift.

Mijn dank gaat ook uit naar de directeur van de Naaldhorst, C Hoedemaker, en de bestuurders Herman Weggen en Martin den Hartog voor de gelegenheid om in de betrokken organisaties een groot deel van het onderzoek te doen.

Ook dank aan mijn collegae specialisten ouderengeneeskunde, ergotherapie en medisch secretariaat voor hun interesse en ondersteuning.

Maar mijn aller grootste dank gaat uit naar mijn vrouw. Arlène, je hebt heelllllllll veell1ll uren alleen gezeten, terwijl ik mij naast mijn drukke werkzaamheden voor De Naaldhorst en later Avoord Zorg en Wonen terugtrok achter mijn PC om onderzoeksplannen en artikelen te schrijven. Vaak zag je dat ik echt uitgeput was, maar dan was jij er met een hapje, een drankje en een arm om mijn schouders, zeggend: er komen nog meer dagen, het hoeft niet af vandaag. Ook mijn 2 zoons 
die vaak een zorgelijke blik hadden en zeiden: pa, doe het wat rustiger aan, wil ik hiervoor bedanken. Want ook jullie waren soms niet mijn eerste prioriteit.

Maar nu is het klaar en kan ik met vrouw, 2 zoons, 2 (schoon)dochters en 3 kleinkinderen gaan genieten van de vrijgekomen tijd. Ik ben trots op het feit dat deze promotie de kroon op mijn werk is en hoop de resultaten met vele professionals te delen. 
O

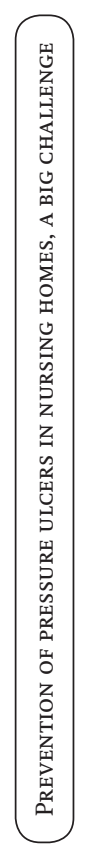


O

146

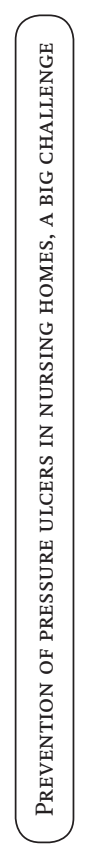




\section{About the author}

Martin van Leen is born in Rotterdam on January $17^{\text {th }}$, 1951. After graduating from secondary school in 1969 at the Franciscus College at Rotterdam he started his medical education at the Erasmus University, where he graduated for his MD in 1977. In 1979 he finished his education as general practitioner at the same University. For the next 6 years he worked as a physician at internal and cardiology wards of 2 hospitals (partly education). At the end of 1985, in expectation of a final 6 months' education in neurology/psychiatry to finish his education as a clinical geriatrician, he started as a general physician and medical manager at nursing home De Naaldhorst, Naaldwijk, The Netherlands. Based on the experience of working at a nursing home he decided to give up the intention to become a clinical geriatrician in 1987. In 1990 he received the registration as an elderly care physician. In 2004 he accepted a job as elderly care physician and medical manager at Avoord Zorg en Wonen, Etten-Leur, The Netherlands.

During the first 10 years of his work in the Naaldhorst he was intrigued by the amount of pressure ulcers, resulting in research about prevention of pressure ulcers and treatment of chronic wounds. In 2004 he had a first talk with prof. dr. Hovius at ErasmusMC, Rotterdam, with the intention to start a PhD study about strategies for the prevention of pressure ulcers. In 2008 he started his $\mathrm{PhD}$ study, first at the department of plastic surgery, ErasmusMC, Rotterdam, later in combination with CAPHRI Research school, department of Health Services Research, Maastricht University, under supervision of prof. dr. JMGA Schols, prof. dr. SER Hovius and dr. RJG Halfens. In 2016 he finished his medical career at Avoord Zorg en Wonen because of reaching the age of retirement.

Martin lives in Rotterdam together with his wife Arlène van Leen-Spuij, enjoying life with his two sons, two daughters (in law) and three grandchildren.

At last he wants to give an advice to future $\mathrm{PhD}$ students: Before starting a PhD study, make sure to save special time weekly, as a part of your job. To realise a $\mathrm{PhD}$ study next to a full-time job is almost impossible. 
O

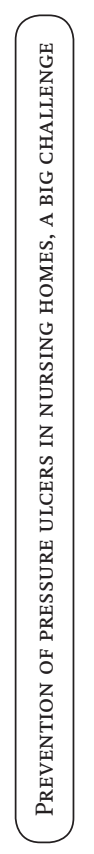




\section{LIST OF PUBLICATIONS AND PRESENTATIONS}


O

150

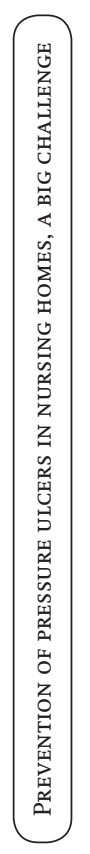




\title{
List of publications and presentations
}

\author{
International publications
}

\section{6}

Thesis article: Van Leen MWF, Schols JMGA, Hovius SER, Halfens RJG. Which pressure ulcer preventive measures are used in Dutch nursing homes? A longitudinal study from 2005-2014. Ostomy Wound Management, accepted January 2017

Thesis article: Van Leen MWF, Halfens RJG, Hovius SER, Schols JMGA. Preventive effect of a skin interface microclimate regulating system on pressure ulcer development. A multicenter, prospective, controlled randomized clinical trial in Dutch nursing homes. Advances in Skin \&Wound Care, accepted October 2016

\section{5}

van Leen MWF, Schols JMGA. Pressure relief, visco-elastic foam with inflated air? A pilot study in a Dutch nursing home. Healthcare 2015,3,78-83;doi:10.3390/ healthcare 3010078

\section{4}

Thesis article: van Leen MWF, Schols JMGA, Hovius SER, Halfens RJG. The Effect of a Simple 3-step Pressure Relieving Strategy for Preventing Pressure Ulcers: $\mathrm{n}$ Explorative Longitudinal Study From 2002-2011. WOUNDS 2014;26(10):285-292

Leen van M.W.F., Rondas A.A.L.M., Neyens J.C.L., Cutting K.F., Schols J.M.G.A. Influence of superabsorbent dressings on non-healing ulcers: a multicenter case series from the Netherlands and the UK. Journal of Wound Care, Vol. 23: Issue. 11: Pages. 543-550. doi: 10.12968/jowc.2014.23.11.543.

\section{3}

Schols, J., Neyens, J., Rondas, A. \& Leen, M. van (2013). The effects of an arginineenriched oral nutritional supplement on chronic wound healing in non-malnourished patients: A multicenter case study in the Netherlands. EWMA Journal, 13 (2), 32-33.

Neyens, J.C.L., Rondas, A.A.L.M., Leen, M.W.F. van \& Schols, J.M.G.A. (2013). Effects of an arginine-enriched oral nutritional supplement on the healing of chronic wounds in non-malnourished patients: a multicenter case study in the Netherlands. In Enthusiasm complements Experience and Evidence in Pressure Ulcer Management. Vienna. 
2011

Thesis article: van Leen M, Hovius S, Neyens J, Halfens R, Schols J. Pressure relief, cold foam or static air? A single centre, prospective, controlled randomized clinical trial in a Dutch nursing home. J Tissue Viability. 2011;20(1);30- 34.

2010

Thesis article: van Leen M, Hovius S, Neyens J, Halfens R, Schols J. Pressure relief, visco-elastic foam or static air? A single centre, prospective, cross-over randomized clinical trial in a Dutch nursing home. WOUNDS. 2013;25(10)287-292.

van Anholt R.D., Sobotka L., Meijer E.P., Topinkova, E.,van Leen M.W.F., Schols J.M.G.A. Specific nutritional support accelerates pressure ulcer healing and reduces wound care intensity in non-malnourished patients. Nutrition September 2010; Volume 26, issue 9: 867-872

2007

Van Leen M.W.F., van der Eijk I, Schols J.M.G.A. Prevention of NSAID gastropathy in elderly patients. An observational study in general practice and nursing homes. Age and Ageing 2007;36:414-418

2001

Müller E, van Leen M.W.F., Bergemann R. Economi evaluation of collagenescontaining Ointment and hydrocolloid dressing in the treatment of pressure ulcers. Pharmacoeconomics 2001;19(12):1209-1216

\section{National publications}

2016

Halfens R.J.G., Rijcken S., van Leen M.W.F., Schols J.M.G.A. Meten van de kwaliteit van de basiszorg. Tijdschrift voor ouderengeneeskunde 2016;(6) http://www.verenso. nl/tijdschrift-voor-ouderengeneeskunde/algemeen/\#.WHDxe_krKUk

2015

van Leen M.W.F. Open of 'gesloten' woonzorgcentra voor cliënten met dementie. Tijdschrift voor ouderengeneeskunde 2015;(3) http://www.verenso.nl/tijdschriftvoor-ouderengeneeskunde/algemeen/\#.WHDxe_krKUk1 
Neyens J.C.L., Rondas A.A.L.M., Leen M.W.F, Schols J.M.G.A. Effecten van een met arginine verrijkt oraal voedingssupplement op de genezing van complexe wonden bij niet-ondervoede patiënten: een multicenter case studie in Nederland. WCS NIEUWS 2014; 30(4): 21-22.

2013

van Leen M.W.F., ten Cate H. Nieuwe orale antistollingsmiddelen, winst of verlies. Tijdschrift voor ouderengeneeskunde 2013; nr 2:76-79

2010

Rondas A.A.L.M. \& Leen M.W.F. van (2012). Wondbehandeling in het verpleeghuis: nieuwe ontwikkelingen en de rol van technologie. Tijdschrift voor Ouderengeneeskunde, 37 (5), 258-259.

van Leen M.W.F., Schols J.M.G.A. Een nieuwe richtlijn hartfalen, winst? Tijdschrift voor ouderengeneeskunde. 2010; $\mathrm{nr}$ 4:127-129

van Leen M.W.F. Cardiovasculaire problemen in het verpleeghuis. Tijdschrift voor ouderengeneeskunde. 2010; $\mathrm{nr}$ 4:137-142

van Leen M.W.F., Brouwers K., van der Meer F., ten Cate H. Nieuwe orale antistollingsmiddelen, winst of verlies? Tijdschrift voor ouderengeneeskunde. 2010; nr 4:159-161

van Dijk R., van Leen M.W.F. ICD en AID: wat kunnen we er mee. Tijdschrift voor ouderengeneeskunde. 2010; nr 4:162-163

2007

van Leen M.W.F. Zilverapplicatie in wondbehandeling producten, wel of niet toepassen? Nederlands tijdschrift voor wondverzorging. 2007 (9);14-17

1997

van Leen M.W.F. Behandeling van chronische wonden. Patient Care. jan 1997;25-31

\section{4}

van Leen M.W.F. Collagenase, hulpmiddel bij decubitus wonden. Tijdschrift voor Therapie, Geneesmiddel en onderzoek. 1994, no 8, 2-7 van Leen M.W.F. Decubitus. De Papieren visite. 1994, $\mathrm{nr}$ 18, 2-4 


\section{International presentations:}

van Leen M.W.F. Effect of collagenase, oral presentation at the joint meeting of ETRS and AWC, Amsterdam May1994

van Leen M.W.F., Smit HJ. Effects of a new cellulose dressing, oral presentation at the EWMA, Prague, May 2006

van Leen M.W.F. Effects of Ph5MB, oral presentation at the EWMA, Prague, May 2006

Neyens J.C.L., Rondas A.A.L.M., Leen M.W.F. van \& Schols J.M.G.A. (2012). Effect of a specific nutritional support for wound healing in patients with leg ulcers, diabetic foot ulcers or pressure ulcers. In European Pressure Advisory Panel EPUAP. Cardiff, 154 Wales.

van Leen MWF, Schols JMGA, Hovius SER, Halfens RJG. (2012) The Effect of a Simple 3-step Pressure Relieving Strategy for Preventing Pressure Ulcers, a longitudinal study from 2002-2011. In European Pressure Advisory Panel EPUAP. Cardiff, Wales.

van Leen MWF, Schols JMGA, Hovius SER, Halfens RJG. (2014) The Effect of a Simple 3-step Pressure Relieving Strategy for Preventing Pressure Ulcers, a longitudinal study from 2002-2011. In European Pressure Advisory Panel EPUAP. Stockholm, Sweden

Neyens J.C.L., Rondas A.A.L.M., Leen van M.W.F., Cereda A., Schols J.M.G.A. (2015) Effects of an arginine-enriched oral nutritional supplement on the healing of chronic wounds in non-malnourished patients; a multicentre case series from the Netherlands. Oral presentation at EWMA Conference in London, Great Brittan.

van Leen MWF, Schols JMGA, Hovius SER, Halfens RJG. (2016) The Effect of a Simple 3-step Pressure Relieving Strategy for Preventing Pressure Ulcers, a longitudinal study from 2002-2011.

Oral presentation in Nursing Home Research International Working Group, Barcelona

\section{National presentations:}

van Leen M.W.F. Preventie van decubitus. WCS congres Utrecht nov.2001. 
van Leen M.W.F. Zin en onzin van zilver bij wondbehandeling. WCS congres Utrecht nov. 2009

van Leen M.W.F. Inzet van decubitussystemen WCS congres Utrecht nov. 2013

van Leen M.W.F. Preventie van decubitus, zinvol of niet .WCS congres Utrecht nov. 2015

van Leen M.W.F. Effectiviteit van statische lucht. Decubituscongres Limburg Kerkrade nov. 2006

van Leen M.W.F. Zin en onzin van zilverbehandeling. Decubituscongres Limburg Kerkrade nov. 2008

van Leen M.W.F. Druk, schuif en wrijvingskrachten. Decubituscongres Limburg Kerkrade nov. 2010

van Leen M.W.F. Veranderen we de bodem of de matras. Decubituscongres Limburg Kerkrade nov. 2010

van Leen M.W.F. Van complexe naar simpele preventie van decubitus. Decubituscongres Limburg Kerkrade nov. 2012

van Leen M.W.F. Verminderen van druk en wrijvingskrachten en reguleren van het microklimaat, zinvol of niet. Landelijk decubituscongres Venlo nov. 2016.

van Leen M.W.F. Invloed van Sorbion® Sana verband op complexe slecht genezende ulcera cruris; een multicenter case studie in Nederland. Dutch NOVW wound congress Ede 2013

van Leen M.W.F. Huidbehandeling bij de oudere patiënt. National Skin therapists congress Ede 2015

van Leen M.W.F. van, Rondas A.A.L.M. (2012). Wondbehandeling in het verpleeghuis. Verenso jubileum congres, Rotterdam.

van Leen M.W.F., Rondas A.A.L.M., Neyens J.C.L., Schols J.M.G.A. (2012). Invloed van Sorbion ${ }^{\circledR}$ Sana verband op complexe slecht genezende ulcera cruris; een multicenter case studie in Nederland. In Verenso jubileumcongres, Rotterdam. 


\section{Poster presentations 2004-2012}

The effect of a simple 3-step pressure relieving strategy for prevention of pressure ulcers; a longitudinal study from 2002-2011, EWMA Wien 2012

van Leen, M.W.F., Cutting, K., Rondas, A.A.L.M., Neyens, J.C.L. \& Schols, J.M.G.A. (2012). Influence of dressings on non-healing wounds: a multi-center case study in England and the Netherlands. In Vol. 12. EWMA Journal (pp. 85).

van Leen, M.W.F., Cutting, K., Rondas, A.A.L.M., Neyens, J. \& Schols, J.M.G.A. (2012). Influence of Sorbion dressing (Sachet $S$ or Sana) on non-healing wounds; a multicenter case study in England and the Netherlands. EWMA Journal, 12 (suppl. 2), 164.

156 Neyens, J.C.L., Rondas, A.A.L.M., Leen, M. van, Cutting, K. \& Schols, J.M.G.A. (2012). Influence of Sorbion® Sana dressings on non-healing leg ulcers; a multi-center case study in the Netherlands and the United Kingdom . In European Wound Management Association (EWMA).

van Leen, M.W.F.v Schols, J.M.G.A., Neyens, J.C.L. \& Rondas, A.A.L.M. (2012). Sorbion® and Sana: a case study. In EWMA conference. Vienna, Austria.

van Leen M.W.F., Heymans H., Klebach M., Hofman Z., van de Looverbosch D. Safe use of a new nutritionally improved tube feet. Espen congress Nice 2010

van Leen M.W.F., van der Eijk I, Schook E. Gastropathy in elderly patients. An observational study in the Netherlands. International congress of rheumatic disorders Norway 2006

van Leen M.W.F. A prospective randomized study in recalcitrant pressure ulcers with polyhydrated ionogens is feasible, $2^{\text {nd }}$ congress of the World Union of Wound Healing Societies, Paris, May 2004. 